\title{
On Positive Solutions of a Fourth Order Nonlinear Neutral Delay Difference Equation
}

\author{
Zeqing Liu, ${ }^{1}$ Xiaoping Zhang, ${ }^{1}$ Shin Min Kang, ${ }^{2}$ and Young Chel Kwun ${ }^{3}$ \\ ${ }^{1}$ Department of Mathematics, Liaoning Normal University, Dalian, Liaoning 116029, China \\ ${ }^{2}$ Department of Mathematics and RINS, Gyeongsang National University, Jinju 660-701, Republic of Korea \\ ${ }^{3}$ Department of Mathematics, Dong-A University, Pusan 614-714, Republic of Korea
}

Correspondence should be addressed to Young Chel Kwun; yckwun@dau.ac.kr

Received 19 August 2013; Accepted 17 December 2013; Published 4 March 2014

Academic Editor: Adem Kılıçman

Copyright (C) 2014 Zeqing Liu et al. This is an open access article distributed under the Creative Commons Attribution License, which permits unrestricted use, distribution, and reproduction in any medium, provided the original work is properly cited.

The existence results of uncountably many bounded positive solutions for a fourth order nonlinear neutral delay difference equation are proved by means of the Krasnoselskii's fixed point theorem and Schauder's fixed point theorem. A few examples are included.

\section{Introduction}

In the past few decades, the researchers [1-31] and others studied oscillation, asymptotic behavior, and solvability for a lot of second and third order nonlinear difference equations, some of which are as follows:

$$
\begin{aligned}
& \Delta\left(a_{n} \Delta\left(x_{n}+p x_{n-\tau}\right)\right)+F\left(n+1, x_{n+1-\sigma}\right)=0, \quad n \geq 1, \\
& \Delta\left(a_{n} \Delta\left(x_{n}+b x_{n-\tau}\right)\right) \\
& +f\left(n, x_{n-d_{1 n}}, \ldots, x_{n-d_{k n}}\right)=c_{n}, \quad n \geq n_{0}, \\
& \quad \Delta^{3} x_{n}+f\left(n, x_{n}, x_{n-r}\right)=0, \quad n \geq n_{0}, \\
& \Delta\left(a_{n} \Delta^{2}\left(x_{n}+P_{n} x_{n-\tau}\right)\right) \\
& \quad+f\left(n, x_{n-d_{1 n}}, \ldots, x_{n-d_{k n}}\right)=g_{n}, \quad n \geq n_{0}, \\
& \Delta^{2}\left(a_{n} \Delta\left(x_{n}+\gamma_{n} x_{n-\tau}\right)\right)+\Delta^{2} f\left(n, x_{n-d_{1 n}}, \ldots, x_{n-d_{k n}}\right) \\
& +\Delta g\left(n, x_{n-d_{1 n}}, \ldots, x_{n-d_{k n}}\right) \\
& =h\left(n, x_{n-d_{1 n}}, \ldots, x_{n-d_{k n}}\right), \quad n \geq n_{0} .
\end{aligned}
$$

By employing a few famous tools in nonlinear analysis including the nonlinear alternative of Leray-Schauder type, Banach's fixed point theorem, Schauder's fixed point theorem,
Krasnoselskii's fixed point theorem, coincidence degree theory and critical point theory, the authors [3, 4, 9, 13-16, 18, 25, 27,28 and others proved the existence of nonoscillatory solutions, uncountably many bounded nonoscillatory solutions and periodic solutions for the difference equations above, where Lipschitz conditions were used in $[14,16]$. Recently, the authors [32] used the Krasnoselskii's fixed point theorem to obtain $h$-asymptotic stability results about the zero solution for a very general first order nonlinear neutral differential equation with functional delay.

However, to our knowledge, no one studied the following fourth order nonlinear neutral delay difference equation:

$$
\begin{aligned}
& \Delta^{3}\left(a_{n} \Delta\left(x_{n}+\gamma_{n} x_{n-\tau}\right)\right)+\Delta^{3} f\left(n, x_{b_{1 n}}, \ldots, x_{b_{k n}}\right) \\
& \quad+\Delta^{2} g\left(n, x_{c_{1 n}}, \ldots, x_{c_{k n}}\right)+\Delta h\left(n, x_{d_{1 n}}, \ldots, x_{d_{k n}}\right) \\
& \quad+p\left(n, x_{o_{1 n}}, \ldots, x_{o_{k n}}\right)=r_{n}, \quad n \geq n_{0},
\end{aligned}
$$

where $\tau, k \in \mathbb{N}, n_{0} \in \mathbb{N}_{0}, f, g, h, p \in C\left(\mathbb{N}_{n_{0}} \times \mathbb{R}^{k}, \mathbb{R}\right)$, $\left\{a_{n}\right\}_{n \in \mathbb{N}_{n_{0}}}$, and $\left\{\gamma_{n}\right\}_{n \in \mathbb{N}_{n_{0}}}$ are real sequences with $a_{n} \neq 0$ for $n \in$ $\mathbb{N}_{n_{0}}$ and $\left\{b_{l n}, c_{l n}, d_{l n}, o_{l n}: n \in \mathbb{N}_{n_{0}}, l \in\{1,2, \ldots, k\}\right\} \subset \mathbb{Z}$ with

$$
\begin{array}{r}
\lim _{n \rightarrow \infty} b_{l n}=\lim _{n \rightarrow \infty} c_{l n}=\lim _{n \rightarrow \infty} d_{l n}=\lim _{n \rightarrow \infty} o_{l n}=+\infty, \\
l \in\{1,2, \ldots, k\} .
\end{array}
$$


The purpose of this paper is to fill this gap in the literature and to study solvability of (2). Under certain conditions, we prove the existence of uncountably many bounded positive solutions of (2) by means of the Krasnoselskii's fixed point theorem and Schauder's fixed point theorem, respectively. Nine examples are included.

This paper is organized as follows. In Section 2 we present some notations, definitions, and lemmas. In Section 3 we establish nine sufficient conditions which guarantee the existence of uncountably many bounded positive solutions of (2) by using fixed point theorems and new techniques. In Section 4 we give nine examples to illustrate the effectiveness and applications of the results presented in Section 3.

\section{Preliminaries}

Throughout this paper, we assume that $\mathbb{R}=(-\infty,+\infty), \mathbb{Z}, \mathbb{N}$ and $\mathbb{N}_{0}$ stand for the sets of all integers, positive integers, and nonnegative integers, respectively:

$$
\begin{gathered}
\mathbb{N}_{n_{0}}=\left\{n: n \in \mathbb{N} \text { with } n \geq n_{0}\right\}, \quad n_{0} \in \mathbb{N}_{0}, \\
\alpha=\inf \left\{b_{l n}, d_{l n}, c_{l n}, o_{l n}: 1 \leq l \leq k, n \in \mathbb{N}_{n_{0}}\right\}, \\
\beta=\min \left\{n_{0}-\tau, \alpha\right\}, \\
\mathbb{Z}_{\beta}=\{n: n \in \mathbb{Z} \text { with } n \geq \beta\} ;
\end{gathered}
$$

$\Delta$ denotes the forward different operator defined by $\Delta x_{n}=$ $x_{n+1}-x_{n}$ and $\Delta^{i} x_{n}=\Delta\left(\Delta^{i-1} x_{n}\right)$ for $i \in\{2,3,4\}$. Let $l_{\beta}^{\infty}$ denote the Banach space of all bounded sequences on $\mathbb{Z}_{\beta}$ with norm:

$$
\begin{gathered}
\|x\|=\sup _{n \in \mathbb{Z}_{\beta}}\left|x_{n}\right| \quad \text { for } x=\left\{x_{n}\right\}_{n \in \mathbb{Z}_{\beta}} \in l_{\beta}^{\infty}, \\
\Omega_{1}(N, M) \\
=\left\{x=\left\{x_{n}\right\}_{n \in \mathbb{Z}_{\beta}} \in l_{\beta}^{\infty}: N \leq x_{n} \leq M, n \in \mathbb{Z}_{\beta}\right\} ; \\
\Omega_{2 T}(N, M)=\left\{x=\left\{x_{n}\right\}_{n \in \mathbb{Z}_{\beta}} \in l_{\beta}^{\infty}: \frac{N}{\gamma_{n}} \leq x_{n} \leq \frac{M}{\gamma_{n}},\right. \\
\left.n \geq T ; \frac{N}{\gamma_{T}} \leq x_{n} \leq \frac{M}{\gamma_{T}}, \beta \leq n<T\right\} ; \\
\Omega_{3 T}(N, M)=\left\{x=\left\{x_{n}\right\}_{n \in \mathbb{Z}_{\beta}} \in l_{\beta}^{\infty}: \frac{-N}{\gamma_{n}} \leq x_{n} \leq \frac{-M}{\gamma_{n}},\right. \\
\left.n \geq T ; \frac{-N}{\gamma_{T}} \leq x_{n} \leq \frac{-M}{\gamma_{T}}, \beta \leq n<T\right\} .
\end{gathered}
$$

Obviously, $\Omega_{1}(N, M), \Omega_{2 T}(N, M)$, and $\Omega_{3 T}(N, M)$, are closed bounded and convex subsets of $l_{\beta}^{\infty}$ for any $M>N>0$.

By a solution of (2), we mean a real sequence $\left\{x_{n}\right\}_{n \in \mathbb{Z}_{\beta}}$ with a positive integer $T \geq n_{0}+\tau+|\beta|$ such that (2) is satisfied for all $n \geq T$.
Definition 1 (see [5]). A subset $D$ of $l_{\beta}^{\infty}$ is said to be uniformly Cauchy (or equi-Cauchy) if for every $\varepsilon>0$ there exists a positive integer $K>\beta$ such that

$$
\left|x_{i}-x_{j}\right|<\varepsilon
$$

whenever $i, j>K$ for any $x=\left\{x_{n}\right\}_{n \in \mathbb{Z}_{\beta}} \in l_{\beta}^{\infty}$.

Lemma 2 (see [5]). Each bounded and uniformly Cauchy subset of $l_{\beta}^{\infty}$ is relatively compact.

Lemma 3 (Krasnoselskii's fixed point theorem). Let $X$ be a Banach space, let $D$ be a bounded closed convex subset of $X$, and let $S, G$ be mappings from $D$ into $X$ such that $S x+G y \in D$ for every pair $x, y \in D$. If $S$ is a contraction and $G$ is completely continuous, then the equation

$$
S x+G x=x
$$

has a solution in $D$.

Lemma 4 (Schauder's fixed point theorem). Let $D$ be a nonempty closed convex subset of a Banach space $X, T: D \rightarrow$ $D$ continuous, and $f(D)$ relatively compact. Then $f$ has at least one fixed point in $D$.

Lemma 5. Let $\tau \in \mathbb{N}, n_{0} \in \mathbb{N}_{0},\left\{a_{n}\right\}_{n \in \mathbb{N}_{n_{0}}},\left\{b_{n}\right\}_{n \in \mathbb{N}_{n_{0}}}$, and $\left\{c_{n}\right\}_{n \in \mathbb{N}_{n_{0}}}$ be nonnegative sequences. If

$$
\sum_{j=n_{0}}^{\infty} \sum_{s=j}^{\infty} \sum_{t=s}^{\infty} j c_{j} a_{s} b_{t}<+\infty,
$$

then

$$
\begin{aligned}
& \sum_{i=1}^{\infty} \sum_{j=n_{0}+i \tau}^{\infty} \sum_{s=j}^{\infty} \sum_{t=s}^{\infty} c_{j} a_{s} b_{t} \\
& =\sum_{j=n_{0}+\tau}^{\infty} \sum_{s=j}^{\infty} \sum_{t=s}^{\infty}\left[\frac{j-n_{0}}{\tau}\right] c_{j} a_{s} b_{t} \\
& \leq \frac{1}{\tau} \sum_{j=n_{0}+\tau}^{\infty} \sum_{s=j}^{\infty} \sum_{t=s}^{\infty} j c_{j} a_{s} b_{t},
\end{aligned}
$$

where $\left[\left(j-n_{0}\right) / \tau\right]$ denotes the integer part of number $\left(j-n_{0}\right) / \tau$.

Proof. Notice that

$$
\begin{aligned}
\sum_{i=1}^{\infty} \sum_{j=n_{0}+i \tau}^{\infty} \sum_{s=j}^{\infty} \sum_{t=s}^{\infty} c_{j} a_{s} b_{t} \\
=\sum_{j=n_{0}+\tau}^{\infty} \sum_{s=j}^{\infty} \sum_{t=s}^{\infty} c_{j} a_{s} b_{t}+\sum_{j=n_{0}+2 \tau}^{\infty} \sum_{s=j}^{\infty} \sum_{t=s}^{\infty} c_{j} a_{s} b_{t} \\
\quad+\sum_{j=n_{0}+3 \tau}^{\infty} \sum_{s=j}^{\infty} \sum_{t=s}^{\infty} c_{j} a_{s} b_{t}+\cdots
\end{aligned}
$$




$$
\begin{aligned}
= & \sum_{j=n_{0}+\tau}^{n_{0}+2 \tau-1} \sum_{s=j}^{\infty} \sum_{t=s}^{\infty}\left[\frac{j-n_{0}}{\tau}\right] c_{j} a_{s} b_{t} \\
& +\sum_{j=n_{0}+2 \tau} \sum_{s=j}^{n_{0}+3 \tau-1} \sum_{t=s}^{\infty}\left[\frac{j-n_{0}}{\tau}\right] c_{j} a_{s} b_{t} \\
& +\sum_{j=n_{0}+3 \tau} \sum_{s=j}^{n_{0}+4 \tau-1} \sum_{t=s}^{\infty}\left[\frac{j-n_{0}}{\tau}\right] c_{j} a_{s} b_{t}+\cdots \\
= & \sum_{j=n_{0}+\tau}^{\infty} \sum_{s=j}^{\infty} \sum_{t=s}^{\infty}\left[\frac{j-n_{0}}{\tau}\right] c_{j} a_{s} b_{t} \\
\leq & \frac{1}{\tau} \sum_{j=n_{0}+\tau}^{\infty} \sum_{s=j}^{\infty} \sum_{t=s}^{\infty} j c_{j} a_{s} b_{t} .
\end{aligned}
$$

That is, (9) holds. This completes the proof.

\section{Existence of Uncountably Many Bounded Positive Solutions}

Now we study the existence of uncountably many bounded positive solutions for (2) by using the Krasnoselskii's fixed point theorem and Schauder's fixed point theorem, respectively.

Theorem 6. Assume that there exist constants $n_{1} \in \mathbb{N}_{n_{0}}, M$, $N$, and $c$ with $M>N>0$ and $c \in[0,(M-N) / 2 M)$ and nonnegative sequences $\left\{W_{n}\right\}_{n \in \mathbb{N}_{n_{0}}},\left\{P_{n}\right\}_{n \in \mathbb{N}_{n_{0}}},\left\{Q_{n}\right\}_{n \in \mathbb{N}_{n_{0}}}$, and $\left\{R_{n}\right\}_{n \in \mathbb{N}_{n_{0}}}$ satisfying

$$
\left|\gamma_{n}\right| \leq c, \quad \forall n \geq n_{1}
$$

$\left|f\left(n, u_{1}, u_{2}, \ldots, u_{k}\right)\right| \leq W_{n}, \quad\left|g\left(n, u_{1}, u_{2}, \ldots, u_{k}\right)\right| \leq P_{n}$, $\left|h\left(n, u_{1}, u_{2}, \ldots, u_{k}\right)\right| \leq Q_{n}, \quad\left|p\left(n, u_{1}, u_{2}, \ldots, u_{k}\right)\right| \leq R_{n}$, $\forall\left(n, u_{1}, u_{2}, \ldots, u_{k}\right) \in \mathbb{N}_{n_{0}} \times[N, M]^{k}$,

$$
\begin{aligned}
\max \left\{\sum_{t=n_{0}}^{\infty} \frac{W_{t}}{\left|a_{t}\right|}, \sum_{s=n_{0}}^{\infty} \sum_{t=s}^{\infty} \frac{1}{\left|a_{s}\right|}\left[(t-s+1) Q_{t}+P_{t}\right],\right. \\
\\
\left.\sum_{j=n_{0}}^{\infty} \sum_{s=j}^{\infty} \sum_{t=s}^{\infty} \frac{t-s+1}{\left|a_{j}\right|}\left(R_{t}+\left|r_{t}\right|\right)\right\}<+\infty
\end{aligned}
$$

Then (2) possesses uncountably many bounded positive solutions in $\Omega_{1}(N, M)$.
Proof. Set $L \in(N+c M, M(1-c))$. It follows from (13) that there exists $T \geq n_{0}+n_{1}+\tau+|\beta|$ sufficiently large such that

$$
\begin{aligned}
\sum_{t=T}^{\infty} \frac{W_{t}}{\left|a_{t}\right|}+\sum_{s=T}^{\infty} \sum_{t=s}^{\infty} \frac{1}{\left|a_{s}\right|}\left[(t-s+1) Q_{t}+P_{t}\right] \\
+\sum_{j=T}^{\infty} \sum_{s=j}^{\infty} \sum_{t=s}^{\infty} \frac{t-s+1}{\left|a_{j}\right|}\left(R_{t}+\left|r_{t}\right|\right) \\
<\min \{M(1-c)-L, L-c M-N\} .
\end{aligned}
$$

Define two mappings $U_{L}$ and $S_{L}: \Omega_{1}(N, M) \rightarrow l_{\beta}^{\infty}$ by

$$
\left(U_{L} x\right)_{n}= \begin{cases}\frac{1}{2} L-\gamma_{n} x_{n-\tau}, & n \geq T \\ \left(U_{L} x\right)_{T}, & \beta \leq n<T\end{cases}
$$

$\left(S_{L} x\right)_{n}$

$$
=\left\{\begin{array}{r}
\frac{1}{2} L+\sum_{t=n}^{\infty} \frac{1}{a_{t}} f\left(t, x_{b_{1 t}}, \ldots, x_{b_{k t}}\right) \\
+\sum_{s=n}^{\infty} \sum_{t=s}^{\infty} \frac{1}{a_{s}}\left[(t-s+1) h\left(t, x_{d_{1 t}}, \ldots, x_{d_{k t}}\right)\right. \\
-\sum_{j=n}^{\infty} \sum_{s=j}^{\infty} \frac{\left.-g\left(t, x_{c_{1 t}}, \ldots, x_{c_{k t}}\right)\right]}{a_{j}}\left[p\left(t, x_{o_{1 t}}, \ldots, x_{o_{k t}}\right)-r_{t}\right], \\
\left(S_{L} x\right)_{T}, \quad n \geq T, \\
\quad \beta \leq n<T
\end{array}\right.
$$

for each $x=\left\{x_{n}\right\}_{n \in \mathbb{Z}_{\beta}} \in \Omega_{1}(N, M)$.

Now we prove that

$$
\begin{gathered}
U_{L} x+S_{L} y \in \Omega_{1}(N, M), \quad \forall x, y \in \Omega_{1}(N, M), \\
\left\|U_{L} x-U_{L} y\right\| \leq c\|x-y\|, \quad \forall x, y \in \Omega_{1}(N, M), \\
\left\|S_{L} x\right\|<M, \quad \forall x, y \in \Omega_{1}(N, M) .
\end{gathered}
$$

In view of (11), (12), and (14)-(16), we conclude that for any $x=\left\{x_{n}\right\}_{n \in \mathbb{Z}_{\beta}}, y=\left\{y_{n}\right\}_{n \in \mathbb{Z}_{\beta}} \in \Omega_{1}(N, M)$, and $n \geq T$,

$$
\begin{aligned}
& \left|\left(U_{L} x\right)_{n}+\left(S_{L} y\right)_{n}-L\right| \\
& =\mid-\gamma_{n} x_{n-\tau}+\sum_{t=n}^{\infty} \frac{1}{a_{t}} f\left(t, y_{b_{1 t}}, \ldots, y_{b_{k t}}\right) \\
& \quad+\sum_{s=n}^{\infty} \sum_{t=s}^{\infty} \frac{1}{a_{s}}\left[(t-s+1) h\left(t, y_{d_{1 t}}, \ldots, y_{d_{k t}}\right)\right. \\
& \quad-\sum_{j=n}^{\infty} \sum_{s=j}^{\infty} \sum_{t=s}^{\infty} \frac{t-s+1}{a_{j}}
\end{aligned}
$$

$$
\times\left[p\left(t, y_{o_{1 t}}, \ldots, y_{o_{\mathrm{kt}}}\right)-r_{t}\right]
$$




$$
\begin{aligned}
& \leq c M+\sum_{t=T}^{\infty} \frac{W_{t}}{\left|a_{t}\right|}+\sum_{s=T}^{\infty} \sum_{t=s}^{\infty} \frac{1}{\left|a_{s}\right|} \\
& \times\left[(t-s+1) Q_{t}+P_{t}\right] \\
& +\sum_{j=T}^{\infty} \sum_{s=j}^{\infty} \sum_{t=s}^{\infty} \frac{t-s+1}{\left|a_{j}\right|}\left(R_{t}+\left|r_{t}\right|\right) \\
& <c M+\min \{M(1-c)-L, L-c M-N\}, \\
& \left\|U_{L} x-U_{L} y\right\|=\sup _{n \in \mathbb{Z}_{\beta}}\left|\left(U_{L} x\right)_{n}-\left(U_{L} y\right)_{n}\right| \\
& =\max \left\{\sup _{T>n \geq \beta}\left|\left(U_{L} x\right)_{n}-\left(U_{L} y\right)_{n}\right|,\right. \\
& \left.\sup _{n \geq T}\left|\left(U_{L} x\right)_{n}-\left(U_{L} y\right)_{n}\right|\right\} \\
& =\sup _{n \geq T}\left(\left|\gamma_{n}\right|\left|x_{n-\tau}-y_{n-\tau}\right|\right) \\
& \leq c\|x-y\|, \\
& \left\|S_{L} x\right\|=\sup _{n \in \mathbb{Z}_{\beta}}\left|\left(S_{L} x\right)_{n}\right| \\
& =\max \left\{\sup _{T>n \geq \beta}\left|\left(S_{L} x\right)_{n}\right|, \sup _{n \geq T}\left|\left(S_{L} x\right)_{n}\right|\right\} \\
& =\sup _{n \geq T} \mid \frac{1}{2} L+\sum_{t=n}^{\infty} \frac{1}{a_{t}} f\left(t, x_{b_{1 t}}, \ldots, x_{\mathrm{b}_{k t}}\right) \\
& +\sum_{s=n}^{\infty} \sum_{t=s}^{\infty} \frac{1}{a_{s}}[(t-s+1) \\
& \times h\left(t, x_{d_{1 t}}, \ldots, x_{d_{k t}}\right) \\
& \left.-g\left(t, x_{c_{1 t}}, \ldots, x_{c_{k t}}\right)\right] \\
& -\sum_{j=n}^{\infty} \sum_{s=j}^{\infty} \sum_{t=s}^{\infty} \frac{t-s+1}{a_{j}}\left[p\left(t, x_{o_{1 t}}, \ldots, x_{o_{k t}}\right)\right. \\
& \left.-r_{t}\right] \\
& \leq \sup _{n \geq T}\left(\frac{1}{2} L+\sum_{t=n}^{\infty} \frac{W_{t}}{\left|a_{t}\right|}+\sum_{s=n}^{\infty} \sum_{t=s}^{\infty} \frac{1}{\left|a_{s}\right|}\left[(t-s+1) Q_{t}\right.\right. \\
& \left.+P_{t}\right] \\
& \left.+\sum_{j=n}^{\infty} \sum_{s=j}^{\infty} \sum_{t=s}^{\infty} \frac{t-s+1}{\left|a_{j}\right|}\left(R_{t}+\left|r_{t}\right|\right)\right) \\
& \leq \frac{1}{2} L+\sum_{t=T}^{\infty} \frac{W_{t}}{\left|a_{t}\right|}+\sum_{s=T}^{\infty} \sum_{t=s}^{\infty} \frac{1}{\left|a_{s}\right|}\left[(t-s+1) Q_{t}+P_{t}\right]
\end{aligned}
$$

which yield that (17) hold.

In order to prove that $S_{L}$ is completely continuous in $\Omega_{1}(N, M)$, we have to show that $S_{L}$ is continuous in $\Omega_{1}(N, M)$ and $S_{L}\left(\Omega_{1}(N, M)\right)$ is relatively compact. Suppose that $\left\{x^{m}\right\}_{m \in \mathbb{N}}$ is an arbitrary sequence in $\Omega_{1}(N, M)$ and $x \in$ $\Omega_{1}(N, M)$ with $\lim _{m \rightarrow \infty} x^{m}=x$, where $x^{m}=\left\{x_{n}^{m}\right\}_{n \in \mathbb{Z}_{\beta}}$ for each $m \in \mathbb{N}$ and $x=\left\{x_{n}\right\}_{n \in \mathbb{Z}_{\beta}}$. With the help of (12), (13), $\lim _{m \rightarrow \infty} x^{m}=x$, and the continuity of $f, g, h$, and $p$, we know that for given $\varepsilon>0$, there exist $T_{1}, T_{2}, T_{3}$, and $T_{4} \in \mathbb{N}$ with $T_{4}>T_{3}>T_{2}>T_{1}>T$ satisfying

$$
\begin{aligned}
& \sum_{t=T_{1}}^{\infty} \frac{W_{t}}{\left|a_{t}\right|}+\sum_{s=T_{1}}^{\infty} \sum_{t=s}^{\infty} \frac{1}{\left|a_{s}\right|}\left[(t-s+1) Q_{t}+P_{t}\right] \\
& +\sum_{j=T_{1}}^{\infty} \sum_{s=j}^{\infty} \sum_{t=s}^{\infty} \frac{t-s+1}{\left|a_{j}\right|} R_{t}<\frac{\varepsilon}{18} \\
& \max \left\{\sum_{t=T_{3}}^{\infty} \frac{t-s+1}{\left|a_{j}\right|} R_{t}: T \leq j \leq T_{1}, j \leq s \leq T_{2}\right\} \\
& <\frac{\varepsilon}{18 T_{1} T_{2}} \text {; } \\
& \max \left\{\sum_{s=T_{2}}^{\infty} \sum_{t=s}^{\infty} \frac{t-s+1}{\left|a_{j}\right|} R_{t}: T \leq j \leq T_{1}\right\}<\frac{\varepsilon}{18 T_{1}} \\
& \max \left\{\sum_{t=T_{2}}^{\infty} \frac{1}{\left|a_{s}\right|}\left[(t-s+1) Q_{t}+P_{t}\right]: T \leq s \leq T_{1}\right\} \\
& <\frac{\varepsilon}{18 T_{1}} \\
& \max \left\{\mid q\left(t, x_{b_{1 t}}^{m}, \ldots, x_{b_{k t}}^{m}\right)\right. \\
& \left.-q\left(t, x_{b_{1 t}}, \ldots, x_{b_{k t}}\right) \mid: q \in\{f, g, h, p\}\right\} \\
& <\frac{\varepsilon}{18 T_{1} T_{2} T_{3}(A+B+E)}, \quad \forall m \geq T_{4}, \quad T \leq t \leq T_{3} \text {, }
\end{aligned}
$$

where

$$
\begin{gathered}
A=\max \left\{\frac{1}{\left|a_{s}\right|}: T \leq s \leq T_{1}\right\}, \\
B=\max \left\{\frac{t-s+1}{\left|a_{s}\right|}: T \leq s \leq T_{1}, s \leq t \leq T_{2}\right\},
\end{gathered}
$$


Abstract and Applied Analysis

5

$$
E=\max \left\{\frac{t-s+1}{\left|a_{j}\right|}: T \leq j \leq T_{1}, j \leq s \leq T_{2}, s \leq t \leq T_{3}\right\} .
$$

By virtue of (16), (19), and (20), we get that

$$
\begin{aligned}
& \left\|S_{L} x^{m}-S_{L} x\right\| \\
& =\sup _{n \in Z_{\beta}}\left|\left(S_{L} x^{m}\right)_{n}-\left(S_{L} x\right)_{n}\right| \\
& =\max \left\{\sup _{T>n \geq \beta}\left|\left(S_{L} x^{m}\right)_{n}-\left(S_{L} x\right)_{n}\right|,\right. \\
& \left.\sup _{n \geq T}\left|\left(S_{L} x^{m}\right)_{n}-\left(S_{L} x\right)_{n}\right|\right\} \\
& =\sup _{n \geq T} \mid \sum_{t=n}^{\infty} \frac{1}{a_{t}} f\left(t, x_{b_{1 t}}^{m}, \ldots, x_{b_{k t}}^{m}\right) \\
& +\sum_{s=n}^{\infty} \sum_{t=s}^{\infty} \frac{1}{a_{s}}\left[(t-s+1) h\left(t, x_{d_{1 t}}^{m}, \ldots, x_{d_{k t}}^{m}\right)\right. \\
& \left.-g\left(t, x_{c_{1 t}}^{m}, \ldots, x_{c_{k t}}^{m}\right)\right] \\
& -\sum_{j=n}^{\infty} \sum_{s=j}^{\infty} \sum_{t=s}^{\infty} \frac{t-s+1}{a_{j}}\left[p\left(t, x_{o_{1 t}}^{m}, \ldots, x_{o_{k t}}^{m}\right)-r_{t}\right] \\
& -\left(\sum_{t=n}^{\infty} \frac{1}{a_{t}} f\left(t, x_{b_{1 t}}, \ldots, x_{b_{k t}}\right)\right. \\
& +\sum_{s=n}^{\infty} \sum_{t=s}^{\infty} \frac{1}{a_{s}}\left[(t-s+1) h\left(t, x_{d_{1 t}}, \ldots, x_{d_{k t}}\right)\right. \\
& \left.-g\left(t, x_{c_{1 t}}, \ldots, x_{c_{k t}}\right)\right] \\
& -\sum_{j=n}^{\infty} \sum_{s=j}^{\infty} \sum_{t=s}^{\infty} \frac{t-s+1}{a_{j}}\left[p\left(t, x_{o_{1 t}}, \ldots, x_{o_{k t}}\right)\right. \\
& \left.\left.-r_{t}\right]\right) \\
& \leq \sum_{t=T}^{\infty} \frac{1}{\left|a_{t}\right|}\left|f\left(t, x_{b_{1 t}}^{m}, \ldots, x_{b_{k t}}^{m}\right)-f\left(t, x_{b_{1 t}}, \ldots, x_{b_{k t}}\right)\right| \\
& +\sum_{s=T}^{\infty} \sum_{t=s}^{\infty} \frac{1}{\left|a_{s}\right|}\left[(t-s+1) \mid h\left(t, x_{d_{1 t}}^{m}, \ldots, x_{d_{k t}}^{m}\right)\right. \\
& -h\left(t, x_{d_{1 t}}, \ldots, x_{d_{k t}}\right) \mid \\
& +\mid g\left(t, x_{c_{1 t}}^{m}, \ldots, x_{c_{k t}}^{m}\right) \\
& \left.-g\left(t, x_{c_{1 t}}, \ldots, x_{c_{k t}}\right) \mid\right]
\end{aligned}
$$

$$
+\sum_{j=T}^{\infty} \sum_{s=j}^{\infty} \sum_{t=s}^{\infty} \frac{t-s+1}{\left|a_{j}\right|} \mid p\left(t, x_{o_{1 t}}^{m}, \ldots, x_{o_{k t}}^{m}\right)
$$

$$
-p\left(t, x_{o_{1 t}}, \ldots, x_{o_{k t}}\right)
$$$$
=\sum_{t=T}^{T_{1}} \frac{1}{\left|a_{t}\right|}\left|f\left(t, x_{b_{1 t}}^{m}, \ldots, x_{b_{k t}}^{m}\right)-f\left(t, x_{b_{1 t}}, \ldots, x_{b_{k t}}\right)\right|
$$$$
+\sum_{t=T_{1}+1}^{\infty} \frac{1}{\left|a_{t}\right|} \mid f\left(t, x_{b_{1 t}}^{m}, \ldots, x_{b_{k t}}^{m}\right)
$$$$
-f\left(t, x_{b_{1 t}}, \ldots, x_{b_{k t}}\right)
$$

$$
\begin{gathered}
+\sum_{s=T}^{T_{1}} \sum_{t=s}^{T_{2}} \frac{1}{\left|a_{s}\right|}\left[(t-s+1) \mid h\left(t, x_{d_{1 t}}^{m}, \ldots, x_{d_{k t}}^{m}\right)\right. \\
-h\left(t, x_{d_{1 t}}, \ldots, x_{d_{k t}}\right) \mid \\
+\mid g\left(t, x_{c_{1 t}}^{m}, \ldots, x_{c_{k t}}^{m}\right) \\
\left.-g\left(t, x_{c_{1 t}}, \ldots, x_{c_{k t}}\right) \mid\right]
\end{gathered}
$$$$
+\sum_{s=T}^{T_{1}} \sum_{t=T_{2}+1}^{\infty} \frac{1}{\left|a_{s}\right|}\left[(t-s+1) \mid h\left(t, x_{d_{1 t}}^{m}, \ldots, x_{d_{k t}}^{m}\right)\right.
$$

$$
-h\left(t, x_{d_{1 t}}, \ldots, x_{d_{k t}}\right)
$$$$
+\mid g\left(t, x_{c_{1 t}}^{m}, \ldots, x_{c_{k t}}^{m}\right)
$$$$
\left.\left.-g\left(t, x_{c_{1 t}}, \ldots, x_{c_{k t}}\right)\right]\right]
$$

$+\sum_{s=T_{1}+1}^{\infty} \sum_{t=s}^{\infty} \frac{1}{\left|a_{s}\right|}\left[(t-s+1) \mid h\left(t, x_{d_{1 t}}^{m}, \ldots, x_{d_{k t}}^{m}\right)\right.$

$$
-h\left(t, x_{d_{1 t}}, \ldots, x_{d_{k t}}\right)
$$$$
+\mid g\left(t, x_{c_{1 t}}^{m}, \ldots, x_{c_{k t}}^{m}\right)
$$$$
\left.-g\left(t, x_{c_{1 t}}, \ldots, x_{c_{k t}}\right) \mid\right]
$$

$$
+\sum_{j=T}^{T_{1}} \sum_{s=j}^{T_{2}} \sum_{t=s}^{T_{3}} \frac{t-s+1}{\left|a_{j}\right|} \mid p\left(t, x_{o_{1 t}}^{m}, \ldots, x_{o_{k t}}^{m}\right)
$$

$$
-p\left(t, x_{o_{1 t}}, \ldots, x_{o_{k t}}\right)
$$$$
+\sum_{j=T}^{T_{1}} \sum_{s=j t=T_{3}+1}^{T_{2}} \sum^{\infty} \frac{t-s+1}{\left|a_{j}\right|} \mid p\left(t, x_{o_{1 t}}^{m}, \ldots, x_{o_{k t}}^{m}\right)
$$$$
-p\left(t, x_{o_{1 t}}, \ldots, x_{o_{k t}}\right) \mid
$$

$$
+\sum_{j=T}^{T_{1}} \sum_{s=T_{2}+1}^{\infty} \sum_{t=s}^{\infty} \frac{t-s+1}{\left|a_{j}\right|} \mid p\left(t, x_{o_{1 t}}^{m}, \ldots, x_{o_{k t}}^{m}\right)
$$

$$
-p\left(t, x_{o_{1 t}}, \ldots, x_{o_{k t}}\right)
$$




$$
\begin{aligned}
& +\sum_{j=T_{1}+1}^{\infty} \sum_{s=j}^{\infty} \sum_{t=s}^{\infty} \frac{t-s+1}{\left|a_{j}\right|} \mid p\left(t, x_{o_{1 t}}^{m}, \ldots, x_{o_{k t}}^{m}\right) \\
& -p\left(t, x_{o_{1 t}}, \ldots, x_{o_{k t}}\right) \\
& \leq \sum_{t=T}^{T_{1}} A\left|f\left(t, x_{b_{1 t}}^{m}, \ldots, x_{b_{k t}}^{m}\right)-f\left(t, x_{b_{1 t}}, \ldots, x_{b_{k t}}\right)\right| \\
& +\sum_{s=T}^{T_{1}} \sum_{t=s}^{T_{2}}\left[B\left|h\left(t, x_{d_{1 t}}^{m}, \ldots, x_{d_{k t}}^{m}\right)-h\left(t, x_{d_{1 t}}, \ldots, x_{d_{k t}}\right)\right|\right. \\
& \left.+A\left|g\left(t, x_{c_{1 t}}^{m}, \ldots, x_{c_{k t}}^{m}\right)-g\left(t, x_{c_{1 t}}, \ldots, x_{c_{k t}}\right)\right|\right] \\
& +\sum_{j=T}^{T_{1}} \sum_{s=j}^{T_{2}} \sum_{t=s}^{T_{3}} E\left|p\left(t, x_{o_{1 t}}^{m}, \ldots, x_{o_{k t}}^{m}\right)-p\left(t, x_{o_{1 t}}, \ldots, x_{o_{k t}}\right)\right| \\
& +2 \sum_{t=T_{1}+1}^{\infty} \frac{W_{t}}{\left|a_{t}\right|}+2 \sum_{s=T}^{T_{1}} \sum_{t=T_{2}+1}^{\infty} \frac{1}{\left|a_{s}\right|}\left[(t-s+1) Q_{t}+P_{t}\right] \\
& +2 \sum_{s=T_{1}+1}^{\infty} \sum_{t=s}^{\infty} \frac{1}{\left|a_{s}\right|}\left[(t-s+1) Q_{t}+P_{t}\right] \\
& +2 \sum_{j=T}^{T_{1}} \sum_{s=j}^{T_{2}} \sum_{t=T_{3}+1}^{\infty} \frac{t-s+1}{\left|a_{j}\right|} R_{t} \\
& +2 \sum_{j=T}^{T_{1}} \sum_{s=T_{2}+1}^{\infty} \sum_{t=s}^{\infty} \frac{t-s+1}{\left|a_{j}\right|} R_{t} \\
& +2 \sum_{j=T_{1}+1}^{\infty} \sum_{s=j}^{\infty} \sum_{t=s}^{\infty} \frac{t-s+1}{\left|a_{j}\right|} R_{t} \\
& <\frac{\varepsilon A\left(T_{1}-T+1\right)}{18 T_{1} T_{2} T_{3}(A+B+E)} \\
& +\frac{\varepsilon(B+A)\left(T_{1}-T+1\right)\left(T_{2}-T+1\right)}{18 T_{1} T_{2} T_{3}(A+B+E)} \\
& +\frac{\varepsilon E\left(T_{1}-T+1\right)\left(T_{2}-T+1\right)\left(T_{3}-T+1\right)}{18 T_{1} T_{2} T_{3}(A+B+E)} \\
& +\frac{2 \varepsilon\left(T_{1}-T+1\right)}{18 T_{1}} \\
& +\frac{2 \varepsilon\left(T_{1}-T+1\right)\left(T_{2}-T+1\right)}{18 T_{1} T_{2}} \\
& +\frac{2 \varepsilon\left(T_{1}-T+1\right)}{18 T_{1}}+\frac{\varepsilon}{18}<\varepsilon \text {, } \\
& \forall m \geq T_{4},
\end{aligned}
$$

which means that $S_{L}$ is continuous in $\Omega_{1}(N, M)$.
Next we prove that $S_{L}\left(\Omega_{1}(N, M)\right)$ is uniformly Cauchy. It follows from (13) that for given $\varepsilon>0$, there exists $V>T$ satisfying

$$
\begin{aligned}
\sum_{t=V}^{\infty} \frac{W_{t}}{\left|a_{t}\right|} & +\sum_{s=V}^{\infty} \sum_{t=s}^{\infty} \frac{1}{\left|a_{s}\right|}\left[(t-s+1) Q_{t}+P_{t}\right] \\
& +\sum_{j=V}^{\infty} \sum_{s=j}^{\infty} \sum_{t=s}^{\infty} \frac{t-s+1}{\left|a_{j}\right|}\left(R_{t}+\left|r_{t}\right|\right)<\frac{\varepsilon}{4} .
\end{aligned}
$$

Using (16) and (22), we know that for any $x=\left\{x_{n}\right\}_{n \in \mathbb{Z}_{\beta}} \in$ $\Omega_{1}(N, M)$ and $m, n \geq V$,

$$
\begin{aligned}
& \left|\left(S_{L} x\right)_{m}-\left(S_{L} x\right)_{n}\right| \\
& =\mid \sum_{t=m}^{\infty} \frac{1}{a_{t}} f\left(t, x_{b_{1 t}}, \ldots, x_{b_{k t}}\right)-\sum_{t=n}^{\infty} \frac{1}{a_{t}} f\left(t, x_{b_{1 t}}, \ldots, x_{b_{k t}}\right) \\
& +\sum_{s=m}^{\infty} \sum_{t=s}^{\infty} \frac{1}{a_{s}}\left[(t-s+1) h\left(t, x_{d_{1 t}}, \ldots, x_{d_{k t}}\right)\right. \\
& \left.-g\left(t, x_{c_{1 t}}, \ldots, x_{c_{k t}}\right)\right] \\
& -\sum_{s=n}^{\infty} \sum_{t=s}^{\infty} \frac{1}{a_{s}}\left[(t-s+1) h\left(t, x_{d_{1 t}}, \ldots, x_{d_{k t}}\right)\right. \\
& \left.-g\left(t, x_{c_{1 t}}, \ldots, x_{c_{k t}}\right)\right] \\
& +\sum_{j=n}^{\infty} \sum_{s=j}^{\infty} \sum_{t=s}^{\infty} \frac{t-s+1}{a_{j}}\left[p\left(t, x_{o_{1 t}}, \ldots, x_{o_{k t}}\right)-r_{t}\right] \\
& -\sum_{j=m}^{\infty} \sum_{s=j}^{\infty} \sum_{t=s}^{\infty} \frac{t-s+1}{a_{j}}\left[p\left(t, x_{o_{1 t}}, \ldots, x_{o_{k t}}\right)-r_{t}\right] \mid \\
& \leq \sum_{t=m}^{\infty} \frac{W_{t}}{\left|a_{t}\right|}+\sum_{t=n}^{\infty} \frac{W_{t}}{\left|a_{t}\right|} \\
& +\sum_{s=m}^{\infty} \sum_{t=s}^{\infty} \frac{1}{\left|a_{s}\right|}\left[(t-s+1) Q_{t}+P_{t}\right] \\
& +\sum_{s=n}^{\infty} \sum_{t=s}^{\infty} \frac{1}{\left|a_{s}\right|}\left[(t-s+1) Q_{t}+P_{t}\right] \\
& +\sum_{j=n}^{\infty} \sum_{s=j}^{\infty} \sum_{t=s}^{\infty} \frac{t-s+1}{\left|a_{j}\right|}\left(R_{t}+\left|r_{t}\right|\right) \\
& +\sum_{j=m}^{\infty} \sum_{s=j}^{\infty} \sum_{t=s}^{\infty} \frac{t-s+1}{\left|a_{j}\right|}\left(R_{t}+\left|r_{t}\right|\right)
\end{aligned}
$$




$$
\begin{aligned}
& \leq 2 \sum_{t=V}^{\infty} \frac{W_{t}}{\left|a_{t}\right|}+2 \sum_{s=V}^{\infty} \sum_{t=s}^{\infty} \frac{1}{\left|a_{s}\right|}\left[(t-s+1) Q_{t}+P_{t}\right] \\
& \quad+2 \sum_{j=V}^{\infty} \sum_{s=j}^{\infty} \sum_{t=s}^{\infty} \frac{t-s+1}{\left|a_{j}\right|}\left(R_{t}+\left|r_{t}\right|\right) \\
& <\varepsilon,
\end{aligned}
$$

which yields that $S_{L}\left(\Omega_{1}(N, M)\right)$ is uniformly Cauchy. It follows from Lemma 2 that $S_{L}\left(\Omega_{1}(N, M)\right)$ is relatively compact. Consequently Lemma 3 guarantees that there exists $x=$ $\left\{x_{n}\right\}_{n \in \mathbb{Z}_{\beta}} \in \Omega_{1}(N, M)$ satisfying $U_{L} x+S_{L} x=x$, which together with (15) and (16) gives that

$$
\begin{array}{r}
x_{n}=L-\gamma_{n} x_{n-\tau}+\sum_{t=n}^{\infty} \frac{1}{a_{t}} f\left(t, x_{b_{1 t}}, \ldots, x_{b_{k t}}\right) \\
+\sum_{s=n}^{\infty} \sum_{t=s}^{\infty} \frac{1}{a_{s}}\left[(t-s+1) h\left(t, x_{d_{1 t}}, \ldots, x_{d_{k t}}\right)\right. \\
\left.-g\left(t, x_{c_{1 t}}, \ldots, x_{c_{k t}}\right)\right] \\
-\sum_{j=n}^{\infty} \sum_{s=j}^{\infty} \sum_{t=s}^{\infty} \frac{t-s+1}{a_{j}}\left[p\left(t, x_{o_{1 t}}, \ldots, x_{o_{k t}}\right)-r_{t}\right], \\
\forall n \geq T,
\end{array}
$$

which yields that

$$
\begin{aligned}
\Delta\left(x_{n}+\gamma_{n} x_{n-\tau}\right)+\frac{1}{a_{n}} f\left(n, x_{b_{1 n}}, \ldots, x_{b_{k n}}\right) \\
=-\sum_{t=n}^{\infty} \frac{1}{a_{n}}\left[(t-n+1) h\left(t, x_{d_{1 t}}, \ldots, x_{d_{k t}}\right)\right. \\
\left.-g\left(t, x_{c_{1 t}}, \ldots, x_{c_{k t}}\right)\right] \\
+\sum_{s=n}^{\infty} \sum_{t=s}^{\infty} \frac{t-s+1}{a_{n}}\left[p\left(t, x_{o_{1 t}}, \ldots, x_{o_{k t}}\right)-r_{t}\right], \\
a_{n} \Delta\left(x_{n}+\gamma_{n} x_{n-\tau}\right)+f\left(n, x_{b_{1 n}}, \ldots, x_{b_{k n}}\right) \\
=-\sum_{t=n}^{\infty}(t-n+1) h\left(t, x_{d_{1 t}}, \ldots, x_{d_{k t}}\right) \\
\quad+\sum_{t=n}^{\infty} g\left(t, x_{c_{1 t}}, \ldots, x_{c_{k t}}\right) \\
\quad+\sum_{s=n}^{\infty} \sum_{t=s}^{\infty}(t-s+1)\left[p\left(t, x_{o_{1 t}}, \ldots, x_{o_{k t}}\right)-r_{t}\right],
\end{aligned}
$$

$\forall n \geq T$, which means that

$$
\begin{aligned}
& \Delta^{3}\left(a_{n} \Delta\left(x_{n}+\gamma_{n} x_{n-\tau}\right)\right)+\Delta^{3} f\left(n, x_{b_{1 n}}, \ldots, x_{b_{k n}}\right) \\
& +\Delta^{2} g\left(n, x_{c_{1 n}}, \ldots, x_{c_{k n}}\right)+\Delta h\left(n, x_{d_{1 n}}, \ldots, x_{d_{k n}}\right) \\
& \quad+p\left(n, x_{o_{1 n}}, \ldots, x_{o_{k n}}\right)=r_{t}, \quad \forall n \geq T ;
\end{aligned}
$$

that is, $x=\left\{x_{n}\right\}_{n \in \mathbb{Z}_{\beta}}$ is a bounded positive solution of (2) in $\Omega_{1}(N, M)$.

Finally we prove that (2) has uncountably many bounded positive solutions in $\Omega_{1}(N, M)$. Let $L_{1}, L_{2} \in(c M+N, M(1-$ c)) and $L_{1} \neq L_{2}$. For each $j \in\{1,2\}$, we conclude similarly that there exist a positive integer $T_{j} \geq n_{0}+n_{1}+\tau+|\beta|$ and two mappings $U_{L_{j}}$ and $S_{L_{j}}$ satisfying (14)-(16), where $L$ and $T$ are replaced by $L_{j}$ and $T_{j}$, respectively, and $U_{L_{j}}+S_{L_{j}}$ has a fixed point $z_{j}=\left\{z_{j n}\right\}_{n \in \mathbb{Z}_{\beta}}$, which is a bounded positive solution (2) in $\Omega_{1}(N, M)$; that is,

$$
\begin{array}{r}
z_{j n}=L_{j}-\gamma_{n} z_{j n-\tau}+\sum_{t=n}^{\infty} \frac{1}{a_{t}} f\left(t, z_{j b_{1 t}}, \ldots, z_{j b_{k t}}\right) \\
+\sum_{s=n}^{\infty} \sum_{t=s}^{\infty} \frac{1}{a_{s}}\left[(t-s+1) h\left(\mathrm{t}, z_{j d_{1 t}}, \ldots, z_{j d_{k t}}\right)\right. \\
\left.-g\left(t, z_{j c_{1 t}}, \ldots, z_{j c_{k t}}\right)\right] \\
-\sum_{j=n}^{\infty} \sum_{s=j}^{\infty} \sum_{t=s}^{\infty} \frac{t-s+1}{a_{j}}\left[p\left(t, z_{j j_{1 t}}, \ldots, z_{j j_{k t}}\right)-r_{t}\right], \\
\forall n \geq T_{j}, \quad j \in\{1,2\} .
\end{array}
$$

Equation (13) ensures that there exists some $T_{3}>$ $\max \left\{T_{1}, T_{2}\right\}$ satisfying

$$
\begin{gathered}
\sum_{t=T_{3}}^{\infty} \frac{W_{t}}{\left|a_{t}\right|}+\sum_{s=T_{3}}^{\infty} \sum_{t=s}^{\infty} \frac{1}{\left|a_{s}\right|}\left[(t-s+1) Q_{t}+P_{t}\right] \\
+\sum_{j=T_{3}}^{\infty} \sum_{s=j}^{\infty} \sum_{t=s}^{\infty} \frac{t-s+1}{\left|a_{j}\right|} R_{t}<\frac{\left|L_{1}-L_{2}\right|}{4} .
\end{gathered}
$$

Combining (11), (12), (28), and (29), we deduce that for any $n \geq T_{3}$,

$$
\begin{aligned}
\mid z_{1 n}- & z_{2 n} \mid \\
= & \mid L_{1}-L_{2}-\gamma_{n} z_{1 n-\tau}+\gamma_{n} z_{2 n-\tau} \\
& +\sum_{t=n}^{\infty} \frac{1}{a_{t}} f\left(t, z_{1 b_{1 t}}, \ldots, z_{1 b_{k t}}\right) \\
& \quad-\sum_{t=n}^{\infty} \frac{1}{a_{t}} f\left(t, z_{2 b_{1 t}}, \ldots, z_{2 b_{k t}}\right)
\end{aligned}
$$




$$
\begin{aligned}
& +\sum_{s=n}^{\infty} \sum_{t=s}^{\infty} \frac{1}{a_{s}}\left[(t-s+1) h\left(t, z_{1 d_{1 t}}, \ldots, z_{1 d_{k t}}\right)\right. \\
& \left.-g\left(t, z_{1 c_{1 t}}, \ldots, z_{1 c_{k t}}\right)\right] \\
& -\sum_{s=n}^{\infty} \sum_{t=s}^{\infty} \frac{1}{a_{s}}\left[(t-s+1) h\left(t, z_{2 d_{1 t}}, \ldots, z_{2 d_{k t}}\right)\right. \\
& \left.-g\left(t, z_{2 c_{1 t}}, \ldots, z_{2 c_{k t}}\right)\right] \\
& -\sum_{j=n}^{\infty} \sum_{s=j}^{\infty} \sum_{t=s}^{\infty} \frac{t-s+1}{a_{j}}\left[p\left(t, z_{1 o_{1 t}}, \ldots, z_{1 o_{k t}}\right)-r_{t}\right] \\
& +\sum_{j=n}^{\infty} \sum_{s=j}^{\infty} \sum_{t=s}^{\infty} \frac{t-s+1}{a_{j}}\left[p\left(t, z_{2 o_{1 t}}, \ldots, z_{2 o_{k t}}\right)-r_{t}\right] \mid \\
& \geq\left|L_{1}-L_{2}\right|-\left|\gamma_{n}\right|\left|z_{1 n-\tau}-z_{2 n-\tau}\right| \\
& -\sum_{t=n}^{\infty} \frac{1}{a_{t}}\left|f\left(t, z_{1 b_{1 t}}, \ldots, z_{1 b_{k t}}\right)-f\left(t, z_{2 b_{1 t}}, \ldots, z_{2 b_{k t}}\right)\right| \\
& -\sum_{s=n}^{\infty} \sum_{t=s}^{\infty} \frac{1}{a_{s}}\left[(t-s+1) \mid h\left(t, z_{1 d_{1 t}}, \ldots, z_{1 d_{k t}}\right)\right. \\
& -h\left(t, z_{2 d_{1 t}}, \ldots, z_{2 d_{k t}}\right) \mid \\
& +\mid g\left(t, z_{1 c_{1 t}}, \ldots, z_{1 c_{k t}}\right) \\
& \left.-g\left(t, z_{2 c_{1 t}}, \ldots, z_{2 c_{k t}}\right) \mid\right] \\
& -\sum_{j=n}^{\infty} \sum_{s=j}^{\infty} \sum_{t=s}^{\infty} \frac{t-s+1}{a_{j}} \mid p\left(t, z_{1 o_{1 t}}, \ldots, z_{1 o_{k t}}\right) \\
& -p\left(t, z_{2 o_{1 t}}, \ldots, z_{2 o_{k t}}\right) \\
& \geq\left|L_{1}-L_{2}\right|-c\left\|z_{1}-z_{2}\right\| \\
& -2\left(\sum_{t=n}^{\infty} \frac{W_{t}}{\left|a_{t}\right|}+\sum_{s=n}^{\infty} \sum_{t=s}^{\infty} \frac{1}{\left|a_{s}\right|}\left[(t-s+1) Q_{t}+P_{t}\right]\right. \\
& \left.+\sum_{j=n}^{\infty} \sum_{s=j}^{\infty} \sum_{t=s}^{\infty} \frac{t-s+1}{\left|a_{j}\right|} R_{t}\right) \\
& \geq\left|L_{1}-L_{2}\right|-c\left\|z_{1}-z_{2}\right\| \\
& -2\left(\sum_{t=T_{3}}^{\infty} \frac{W_{t}}{\left|a_{t}\right|}+\sum_{s=T_{3}}^{\infty} \sum_{t=s}^{\infty} \frac{1}{\left|a_{s}\right|}\left[(t-s+1) Q_{t}+P_{t}\right]\right. \\
& \left.+\sum_{j=T_{3}}^{\infty} \sum_{s=j}^{\infty} \sum_{t=s}^{\infty} \frac{t-s+1}{\left|a_{j}\right|} R_{t}\right) \\
& >\frac{\left|L_{1}-L_{2}\right|}{2}-c\left\|z_{1}-z_{2}\right\| \text {, }
\end{aligned}
$$

which implies that

$$
\left\|z_{1}-z_{2}\right\|>\frac{\left|L_{1}-L_{2}\right|}{2(1+c)}>0 .
$$

That is, $z_{1} \neq z_{2}$. Thus (2) has uncountably many bounded positive solutions in $\Omega_{1}(N, M)$. This completes the proof.

Theorem 7. Assume that there exist constants $n_{1} \in \mathbb{N}_{n_{0}}, M$, $N$, and $c$ with $M>N>0$ and $c \in[0,(M-N) / M)$ and nonnegative sequences $\left\{W_{n}\right\}_{n \in \mathbb{N}_{n_{0}}},\left\{P_{n}\right\}_{n \in \mathbb{N}_{n_{0}}},\left\{Q_{n}\right\}_{n \in \mathbb{N}_{n_{0}}}$, and $\left\{R_{n}\right\}_{n \in \mathbb{N}_{n_{0}}}$ satisfying (12), (13), and

$$
0 \leq \gamma_{n} \leq c, \quad \forall n \geq n_{1}
$$

Then (2) possesses uncountably many bounded positive solutions in $\Omega_{1}(N, M)$.

Proof. Let $L \in(N+c M, M)$. It follows from (13) that there exists $T \geq n_{0}+n_{1}+\tau+|\beta|$ sufficiently large such that

$$
\begin{gathered}
\sum_{t=T}^{\infty} \frac{W_{t}}{\left|a_{t}\right|}+\sum_{s=T}^{\infty} \sum_{t=s}^{\infty} \frac{1}{\left|a_{s}\right|}\left[(t-s+1) Q_{t}+P_{t}\right] \\
+\sum_{j=T}^{\infty} \sum_{s=j}^{\infty} \sum_{t=s}^{\infty} \frac{t-s+1}{\left|a_{j}\right|}\left(R_{t}+\left|r_{t}\right|\right) \\
<\min \{M-L, L-c M-N\} .
\end{gathered}
$$

Let the mappings $U_{L}$ and $S_{L}: \Omega_{1}(N, M) \rightarrow l_{\beta}^{\infty}$ be defined by (15) and (16), respectively. By means of (12), (15), (16), (32), and (33), we infer that for any $x=\left\{x_{n}\right\}_{n \in \mathbb{Z}_{\beta}}, y=\left\{y_{n}\right\}_{n \in \mathbb{Z}_{\beta}} \in$ $\Omega_{1}(N, M)$, and $n \geq T$,

$$
\begin{aligned}
& \left(U_{L} x\right)_{n}+\left(S_{L} y\right)_{n} \\
& =L-\gamma_{n} x_{n-\tau}+\sum_{t=n}^{\infty} \frac{1}{a_{t}} f\left(t, y_{b_{1 t}}, \ldots, y_{b_{k t}}\right) \\
& \quad+\sum_{s=n}^{\infty} \sum_{t=s}^{\infty} \frac{1}{a_{s}}\left[(t-s+1) h\left(t, y_{d_{1 t}}, \ldots, y_{d_{k t}}\right)\right. \\
& \left.\quad-g\left(t, y_{c_{1 t}}, \ldots, y_{c_{k t}}\right)\right] \\
& \quad-\sum_{j=n}^{\infty} \sum_{s=j}^{\infty} \sum_{t=s}^{\infty} \frac{t-s+1}{a_{j}}\left[p\left(t, y_{o_{1 t}}, \ldots, y_{o_{k t}}\right)-r_{t}\right]
\end{aligned}
$$




$$
\begin{aligned}
& \leq L+\sum_{t=T}^{\infty} \frac{W_{t}}{\left|a_{t}\right|}+\sum_{s=T}^{\infty} \sum_{t=s}^{\infty} \frac{1}{\left|a_{s}\right|}\left[(t-s+1) Q_{t}+P_{t}\right] \\
& +\sum_{j=T s=j}^{\infty} \sum_{t=s}^{\infty} \frac{t-s+1}{\left|a_{j}\right|}\left(R_{t}+\left|r_{t}\right|\right) \\
& <L+\min \{M-L, L-c M-N\} \\
& \leq M, \\
& \left(U_{L} x\right)_{n}+\left(S_{L} y\right)_{n} \\
& =L-\gamma_{n} x_{n-\tau}+\sum_{t=n}^{\infty} \frac{1}{a_{t}} f\left(t, y_{b_{1 t}}, \ldots, y_{b_{k t}}\right) \\
& +\sum_{s=n}^{\infty} \sum_{t=s}^{\infty} \frac{1}{a_{s}}\left[(t-s+1) h\left(t, y_{d_{1 t}}, \ldots, y_{d_{k t}}\right)\right. \\
& \left.-g\left(t, y_{c_{1 t}}, \ldots, y_{c_{k t}}\right)\right] \\
& -\sum_{j=n}^{\infty} \sum_{s=j}^{\infty} \sum_{t=s}^{\infty} \frac{t-s+1}{a_{j}}\left[p\left(t, y_{o_{1 t}}, \ldots, y_{o_{k t}}\right)-r_{t}\right] \\
& \geq L-c M-\sum_{t=T}^{\infty} \frac{W_{t}}{\left|a_{t}\right|} \\
& -\sum_{s=T}^{\infty} \sum_{t=s}^{\infty} \frac{1}{\left|a_{s}\right|}\left[(t-s+1) Q_{t}+P_{t}\right] \\
& -\sum_{j=T s=j t=s}^{\infty} \sum^{\infty} \sum^{t} \frac{t-s+1}{\left|a_{j}\right|}\left(R_{t}+\left|r_{t}\right|\right) \\
& >L-c M-\min \{M-L, L-c M-N\} \\
& \geq N
\end{aligned}
$$

that is, $U_{L} x+S_{L} y \in \Omega_{1}(N, M)$ for any $x, y \in \Omega_{1}(N, M)$. The rest of the proof is similar to that of Theorem 6 and is omitted. This completes the proof.

Theorem 8. Assume that there exist constants $n_{1} \in \mathbb{N}_{n_{n}}, M$, $N$, and $c$ with $M>N>0$ and $c \in[0,(M-N) / M)$ and four nonnegative sequences $\left\{W_{n}\right\}_{n \in \mathbb{N}_{n_{0}}},\left\{P_{n}\right\}_{n \in \mathbb{N}_{n_{0}}},\left\{Q_{n}\right\}_{n \in \mathbb{N}_{n_{0}}}$, and $\left\{R_{n}\right\}_{n \in \mathbb{N}_{n_{0}}}$ satisfying (12), (13), and

$$
-c \leq \gamma_{n} \leq 0, \quad \forall n \geq n_{1}
$$

Then (2) possesses uncountably many bounded positive solutions in $\Omega_{1}(N, M)$.
Proof. Let $L \in(N,(1-c) M)$. It follows from (13) that there exists $T \geq n_{0}+n_{1}+\tau+|\beta|$ sufficiently large such that

$$
\begin{gathered}
\sum_{t=T}^{\infty} \frac{W_{t}}{\left|a_{t}\right|}+\sum_{s=T}^{\infty} \sum_{t=s}^{\infty} \frac{1}{\left|a_{s}\right|}\left[(t-s+1) Q_{t}+P_{t}\right] \\
+\sum_{j=T}^{\infty} \sum_{s=j}^{\infty} \sum_{t=s}^{\infty} \frac{t-s+1}{\left|a_{j}\right|}\left(R_{t}+\left|r_{t}\right|\right) \\
<\min \{(1-c) M-L, L-N\} .
\end{gathered}
$$

Let the mappings $U_{L}$ and $S_{L}: \Omega_{1}(N, M) \rightarrow l_{\beta}^{\infty}$ be defined by (15) and (16), respectively. Making use of (12), (15), (16), (35), and (36), we derive that for any $x=\left\{x_{n}\right\}_{n \in \mathbb{Z}_{\beta}}, y=\left\{y_{n}\right\}_{n \in \mathbb{Z}_{\beta}} \in$ $\Omega_{1}(N, M)$ and $n \geq T$,

$$
\begin{aligned}
\left(U_{L} x\right)_{n} & +\left(S_{L} y\right)_{n} \\
= & L-\gamma_{n} x_{n-\tau}+\sum_{t=n}^{\infty} \frac{1}{a_{t}} f\left(t, y_{b_{1 t}}, \ldots, y_{b_{k t}}\right) \\
& +\sum_{s=n}^{\infty} \sum_{t=s}^{\infty} \frac{1}{a_{s}}\left[(t-s+1) h\left(t, y_{d_{1 t}}, \ldots, y_{d_{k t}}\right)\right. \\
& -\sum_{j=n}^{\infty} \sum_{s=j}^{\infty} \sum_{t=s}^{\infty} \frac{t-s+1}{a_{j}}\left[p\left(t, y_{o_{1 t}}, \ldots, y_{o_{k t}}\right)-r_{t}\right] \\
\leq & L+c M+\sum_{t=T}^{\infty} \frac{W_{t}}{\left|a_{t}\right|} \\
& +\sum_{s=T}^{\infty} \sum_{t=s}^{\infty} \frac{1}{\left|a_{s}\right|}\left[(t-s+1) Q_{t}+P_{t}\right] \\
& +\sum_{j=T}^{\infty} \sum_{s=j}^{\infty} \sum_{t=s}^{\infty} \frac{t-s+1}{\left|a_{j}\right|}\left(R_{t}+\left|r_{t}\right|\right) \\
< & \left.\left.L-y_{c_{k t}}\right)\right] \\
\leq & +c M+\min \{(1-c) M-L, L-N\} \\
\left(U_{L} x\right)_{n} & +\left(S_{L} y\right)_{n} \\
&
\end{aligned}
$$




$$
\begin{aligned}
& +\sum_{s=n}^{\infty} \sum_{t=s}^{\infty} \frac{1}{a_{s}}\left[(t-s+1) h\left(t, y_{d_{1 t}}, \ldots, y_{d_{k t}}\right)\right. \\
& \left.-g\left(t, y_{c_{1 t}}, \ldots, y_{c_{k t}}\right)\right] \\
& -\sum_{j=n}^{\infty} \sum_{s=j}^{\infty} \sum_{t=s}^{\infty} \frac{t-s+1}{a_{j}}\left[p\left(t, y_{o_{1 t}}, \ldots, y_{o_{k t}}\right)-r_{t}\right] \\
& \geq L-\sum_{t=T}^{\infty} \frac{W_{t}}{\left|a_{t}\right|}-\sum_{s=T}^{\infty} \sum_{t=s}^{\infty} \frac{1}{\left|a_{s}\right|}\left[(t-s+1) Q_{t}+P_{t}\right] \\
& \quad-\sum_{j=T}^{\infty} \sum_{s=j}^{\infty} \sum_{t=s}^{\infty} \frac{t-s+1}{\left|a_{j}\right|}\left(R_{t}+\left|r_{t}\right|\right) \\
& >L-\min \{(1-c) M-L, L-N\} \\
& \geq N ;
\end{aligned}
$$

that is, $U_{L} x+S_{L} y \in \Omega_{1}(N, M)$ for any $x, y \in \Omega_{1}(N, M)$. The rest of the proof is similar to that of Theorem 6 and is omitted. This completes the proof.

Theorem 9. Assume that there exist constants $n_{1} \in \mathbb{N}_{n_{0}}, M$, $N$, and $c$ with $M>N>0$ and $c>M /(M-N)$ and nonnegative sequences $\left\{W_{n}\right\}_{n \in \mathbb{N}_{n_{0}}},\left\{P_{n}\right\}_{n \in \mathbb{N}_{n_{0}}},\left\{Q_{n}\right\}_{n \in \mathbb{N}_{n_{0}}}$, and $\left\{R_{n}\right\}_{n \in \mathbb{N}_{n_{0}}}$ satisfying (13):

$$
\gamma_{n} \geq c, \quad \forall n \geq n_{1}
$$

$\left|f\left(n, u_{1}, u_{2}, \ldots, u_{k}\right)\right| \leq W_{n}, \quad\left|g\left(n, u_{1}, u_{2}, \ldots, u_{k}\right)\right| \leq P_{n}$ $\left|h\left(n, u_{1}, u_{2}, \ldots, u_{k}\right)\right| \leq Q_{n}, \quad\left|p\left(n, u_{1}, u_{2}, \ldots, u_{k}\right)\right| \leq R_{n}$,

$$
\forall\left(n, u_{1}, u_{2}, \ldots, u_{k}\right) \in \mathbb{N}_{n_{0}} \times\left[0, \frac{M}{c}\right]^{k}
$$

Then (2) possesses uncountably many bounded positive solutions in $l_{\beta}^{\infty}$.

Proof. Let $L \in(N+M / c, M)$. It follows from (13) that there exists an integer $T \geq n_{0}+n_{1}+\tau+|\beta|$ satisfying

$$
\begin{gathered}
\sum_{t=T}^{\infty} \frac{W_{t}}{\left|a_{t}\right|}+\sum_{s=T}^{\infty} \sum_{t=s}^{\infty} \frac{1}{\left|a_{s}\right|}\left[(t-s+1) Q_{t}+P_{t}\right] \\
+\sum_{j=T}^{\infty} \sum_{s=j}^{\infty} \sum_{t=s}^{\infty} \frac{t-s+1}{\left|a_{j}\right|}\left(R_{t}+\left|r_{t}\right|\right) \\
<\min \left\{M-L, L-N-\frac{M}{c}\right\} .
\end{gathered}
$$

Define two mappings $U_{L}$ and $S_{L}: \Omega_{2 T}(N, M) \rightarrow l_{\beta}^{\infty}$ by

$$
\begin{aligned}
& \left(U_{L} x\right)_{n}= \begin{cases}\frac{1}{\gamma_{n+\tau}}\left(L-x_{n+\tau}\right), & n \geq T, \\
\left(U_{L} x\right)_{T}, & \beta \leq n<T\end{cases}
\end{aligned}
$$

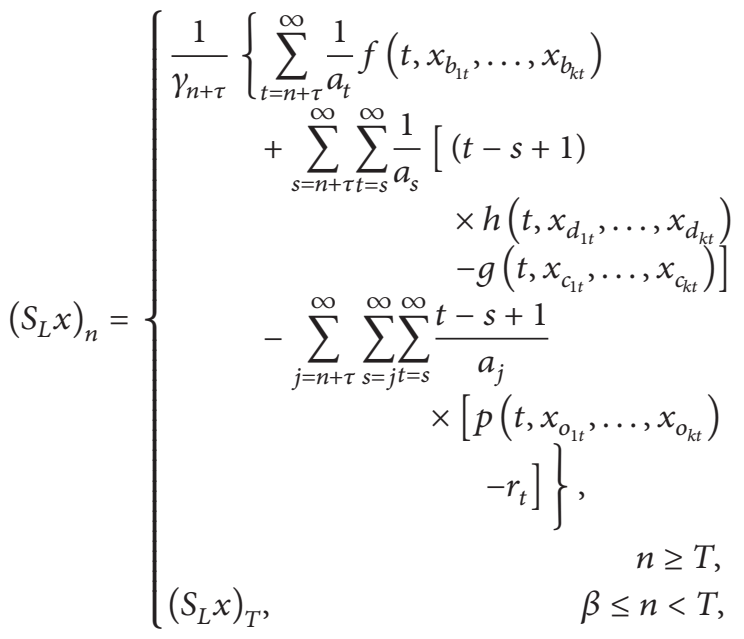

for each $x=\left\{x_{n}\right\}_{n \in \mathbb{Z}_{\beta}} \in \Omega_{2 T}(N, M)$. By means of (13) and (39)-(42), we get that for any $x=\left\{x_{n}\right\}_{n \in \mathbb{Z}_{\beta}}, y=\left\{y_{n}\right\}_{n \in \mathbb{Z}_{\beta}} \in$ $\Omega_{2 T}(N, M)$, and $n \geq T$,

$$
\begin{aligned}
& \left(U_{L} x\right)_{n}+\left(S_{L} y\right)_{n} \\
& =\frac{1}{\gamma_{n+\tau}}\left(L-x_{n+\tau}+\sum_{t=n+\tau}^{\infty} \frac{1}{a_{t}} f\left(t, y_{b_{1 t}}, \ldots, y_{b_{k t}}\right)\right. \\
& +\sum_{s=n+\tau} \sum_{t=s}^{\infty} \frac{1}{a_{s}}\left[(t-s+1) h\left(t, y_{d_{1 t}}, \ldots, y_{d_{k t}}\right)\right. \\
& \quad-\sum_{j=n+\tau}^{\infty} \sum_{s=j}^{\infty} \sum_{t=s}^{\infty} \frac{t-s+1}{a_{j}}\left[p\left(t, y_{o_{1 t}}, \ldots, y_{o_{k t}}\right)\right. \\
&
\end{aligned}
$$

$$
\begin{aligned}
\leq \frac{1}{\gamma_{n+\tau}}( & L-\frac{N}{\gamma_{n+\tau}}+\sum_{t=T+\tau}^{\infty} \frac{W_{t}}{\left|a_{t}\right|} \\
& +\sum_{s=T+\tau}^{\infty} \sum_{t=s}^{\infty} \frac{1}{\left|a_{s}\right|}\left[(t-s+1) Q_{t}+P_{t}\right] \\
& \left.+\sum_{j=T+\tau}^{\infty} \sum_{s=j}^{\infty} \sum_{t=s}^{\infty} \frac{t-s+1}{a_{j}}\left(R_{t}+\left|r_{t}\right|\right)\right)
\end{aligned}
$$




$$
\begin{aligned}
& <\frac{1}{\gamma_{n+\tau}}\left(L+\min \left\{M-L, L-N-\frac{M}{c}\right\}\right) \\
& \leq \frac{M}{\gamma_{n+\tau}}, \\
& \left(U_{L} x\right)_{n}+\left(S_{L} y\right)_{n} \\
& =\frac{1}{\gamma_{n+\tau}}\left(L-x_{n+\tau}+\sum_{t=n+\tau}^{\infty} \frac{1}{a_{t}} f\left(t, y_{b_{1 t}}, \ldots, y_{b_{k t}}\right)\right. \\
& +\sum_{s=n+\tau}^{\infty} \sum_{t=s}^{\infty} \frac{1}{a_{s}}\left[(t-s+1) h\left(t, y_{d_{1 t}}, \ldots, y_{d_{k t}}\right)\right. \\
& \left.-g\left(t, y_{c_{1 t}}, \ldots, y_{c_{k t}}\right)\right] \\
& \left.-\sum_{j=n+\tau}^{\infty} \sum_{s=j}^{\infty} \sum_{t=s}^{\infty} \frac{t-s+1}{a_{j}}\left[p\left(t, y_{o_{1 t}}, \ldots, y_{o_{k t}}\right)-r_{t}\right]\right) \\
& \geq \frac{1}{\gamma_{n+\tau}}\left(L-\frac{M}{\gamma_{n+\tau}}-\sum_{t=T+\tau}^{\infty} \frac{W_{t}}{\left|a_{t}\right|}\right. \\
& -\sum_{s=T+\tau}^{\infty} \sum_{t=s}^{\infty} \frac{1}{\left|a_{s}\right|}\left[(t-s+1) Q_{t}+P_{t}\right] \\
& \left.-\sum_{j=T+\tau}^{\infty} \sum_{s=j}^{\infty} \sum_{t=s}^{\infty} \frac{t-s+1}{a_{j}}\left(R_{t}+\left|r_{t}\right|\right)\right) \\
& >\frac{1}{\gamma_{n+\tau}}\left(L-\frac{M}{c}-\min \left\{M-L, L-N-\frac{M}{c}\right\}\right) \\
& \geq \frac{N}{\gamma_{n+\tau}} \text {, }
\end{aligned}
$$

which yield that $U_{L} x+S_{L} y \in \Omega_{2 T}(N, M)$ for any $x, y \in$ $\Omega_{2 T}(N, M)$. By virtue of (38) and (41), we infer that

$$
\begin{aligned}
\left\|U_{L} x-U_{L} y\right\| & =\sup _{n \in \mathbb{Z}_{\beta}}\left|\left(U_{L} x\right)_{n}-\left(U_{L} y\right)_{n}\right| \\
& =\max \left\{\sup _{T>n \geq \beta}\left|\left(U_{L} x\right)_{n}-\left(U_{L} y\right)_{n}\right|,\right. \\
& \left.\sup _{n \geq T}\left|\left(U_{L} x\right)_{n}-\left(U_{L} y\right)_{n}\right|\right\} \\
& =\sup _{n \geq T}\left(\frac{1}{\left|\gamma_{n+\tau}\right|}\left|x_{n+\tau}-y_{n+\tau}\right|\right) \\
& \leq \frac{1}{c}\|x-y\| ;
\end{aligned}
$$

that is, $U_{L}$ is a contraction in $\Omega_{2 T}(N, M)$ because $c>M /(M-$ $N)>1$.

In order to prove that $S_{L}$ is completely continuous in $\Omega_{2 T}(N, M)$, we have to show that $S_{L}$ is continuous in $\Omega_{2 T}(N, M)$ and $S_{L}\left(\Omega_{2 T}(N, M)\right.$ is relatively compact. Suppose that $\left\{x^{m}\right\}_{m \in \mathbb{N}}$ is an arbitrary sequence in $\Omega_{2 T}(N, M)$ and $x \in$ $\Omega_{2 T}(N, M)$ with $\lim _{m \rightarrow \infty} x^{m}=x$, where $x^{m}=\left\{x_{n}^{m}\right\}_{n \in \mathbb{Z}_{\beta}}$ for each $m \in \mathbb{N}$ and $x=\left\{x_{n}\right\}_{n \in \mathbb{Z}_{\beta}}$. On account of (13), (39), $\lim _{m \rightarrow \infty} x^{m}=x$, and the continuity of $f, g, h$, and $p$, we obtain that for given $\varepsilon>0$, there exist $T_{1}, T_{2}, T_{3}$, and $T_{4} \in \mathbb{N}$ with $T_{4}>T_{3}>T_{2}>T_{1}>T$ satisfying

$$
\begin{gathered}
\sum_{t=T_{1}+\tau}^{\infty} \frac{W_{t}}{\left|a_{t}\right|}+\sum_{s=T_{1}+\tau}^{\infty} \sum_{t=s}^{\infty} \frac{1}{\left|a_{s}\right|}\left[(t-s+1) Q_{t}+P_{t}\right] \\
+\sum_{j=T_{1}+\tau}^{\infty} \sum_{s=j}^{\infty} \sum_{t=s}^{\infty} \frac{t-s+1}{\left|a_{j}\right|} R_{t}<\frac{\varepsilon c}{18}
\end{gathered}
$$

$\max \left\{\sum_{t=T_{3}+\tau}^{\infty} \frac{t-s+1}{\left|a_{j}\right|} R_{t}: T \leq j \leq T_{1}+\tau, \quad j \leq s \leq T_{2}+\tau\right\}$ $<\frac{\varepsilon \mathcal{C}}{18 T_{1} T_{2}}$;

$\max \left\{\sum_{s=T_{2}+\tau}^{\infty} \sum_{t=s}^{\infty} \frac{t-s+1}{\left|a_{j}\right|} R_{t}: T \leq j \leq T_{1}+\tau\right\}$ $<\frac{\varepsilon \mathcal{E c}}{18 T_{1}}$;

$$
\begin{gathered}
\max \left\{\sum_{t=T_{2}+\tau}^{\infty} \frac{1}{\left|a_{s}\right|}\left[(t-s+1) Q_{t}+P_{t}\right]\right. \\
\left.: T \leq s \leq T_{1}+\tau\right\}<\frac{\varepsilon c}{18 T_{1}}
\end{gathered}
$$

$$
\begin{aligned}
\max & \left\{\mid q\left(t, x_{b_{1 t}}^{m}, \ldots, x_{b_{k t}}^{m}\right)\right. \\
& \left.-q\left(t, x_{b_{1 t}}, \ldots, x_{b_{k t}}\right) \mid: q \in\{f, g, h, p\}\right\} \\
< & \frac{\varepsilon c}{18 T_{1} T_{2} T_{3}(A+B+E)}, \quad \forall m \geq T_{4}, \quad T \leq t \leq T_{3},
\end{aligned}
$$

where

$$
\begin{gathered}
A=\max \left\{\frac{1}{\left|a_{s}\right|}: T+\tau \leq s \leq T_{1}+\tau\right\}, \\
B=\max \left\{\frac{t-s+1}{\left|a_{s}\right|}: T+\tau \leq s \leq T_{1}+\tau, s \leq t \leq T_{2}+\tau\right\}, \\
E=\max \left\{\frac{t-s+1}{\left|a_{j}\right|}: T+\tau \leq j \leq T_{1}+\tau,\right. \\
\left.j \leq s \leq T_{2}+\tau, s \leq t \leq T_{3}+\tau\right\} .
\end{gathered}
$$


12

Abstract and Applied Analysis

It follows from (42)-(46) that

$$
\begin{aligned}
& \left\|S_{L} x^{m}-S_{L} x\right\| \\
& =\sup _{n \in \mathbb{Z}_{\beta}}\left|\left(S_{L} x^{m}\right)_{n}-\left(S_{L} x\right)_{n}\right| \\
& =\max \left\{\sup _{T>n \geq \beta}\left|\left(S_{L} x^{m}\right)_{n}-\left(S_{L} x\right)_{n}\right|,\right. \\
& \left.\sup _{n \geq T}\left|\left(S_{L} x^{m}\right)_{n}-\left(S_{L} x\right)_{n}\right|\right\} \\
& =\sup _{n \geq T} \mid \frac{1}{\gamma_{n+\tau}}\left(\sum_{t=n+\tau}^{\infty} \frac{1}{a_{t}} f\left(t, x_{b_{1 t}}^{m}, \ldots, x_{b_{k t}}^{m}\right)\right. \\
& +\sum_{s=n+\tau}^{\infty} \sum_{t=s}^{\infty} \frac{1}{a_{s}}\left[(t-s+1) h\left(t, x_{d_{1 t}}^{m}, \ldots, x_{d_{k t}}^{m}\right)\right. \\
& \left.-g\left(t, x_{c_{1 t}}^{m}, \ldots, x_{c_{k t}}^{m}\right)\right] \\
& \left.-\sum_{j=n+\tau}^{\infty} \sum_{s=j}^{\infty} \sum_{\mathrm{t}=s}^{\infty} \frac{t-s+1}{a_{j}}\left[p\left(t, x_{o_{1 t}}^{m}, \ldots, x_{o_{k t}}^{m}\right)-r_{t}\right]\right) \\
& -\frac{1}{\gamma_{n+\tau}}\left(\sum_{t=n+\tau}^{\infty} \frac{1}{a_{t}} f\left(t, x_{b_{1 t}}, \ldots, x_{b_{k t}}\right)\right. \\
& +\sum_{s=n+\tau}^{\infty} \sum_{t=s}^{\infty} \frac{1}{a_{s}}\left[(t-s+1) h\left(t, x_{d_{1 t}}, \ldots, x_{d_{k t}}\right)\right. \\
& \left.-g\left(t, x_{c_{1 t}}, \ldots, x_{c_{k t}}\right)\right] \\
& -\sum_{j=n+\tau s=j}^{\infty} \sum_{t=s}^{\infty} \frac{t-s+1}{a_{j}} \\
& \left.\times\left[p\left(t, x_{o_{1 t}}, \ldots, x_{o_{k t}}\right)-r_{t}\right]\right) \\
& \leq \frac{1}{c}\left(\sum_{t=T+\tau}^{\infty} \frac{1}{\left|a_{t}\right|} \mid f\left(t, x_{b_{1 t}}^{m}, \ldots, x_{b_{k t}}^{m}\right)\right. \\
& -f\left(t, x_{b_{1 t}}, \ldots, x_{b_{k t}}\right) \\
& +\sum_{s=T+\tau}^{\infty} \sum_{t=s}^{\infty} \frac{1}{\left|a_{s}\right|}\left[(t-s+1) \mid h\left(t, x_{d_{1 t}}^{m}, \ldots, x_{d_{k t}}^{m}\right)\right. \\
& -h\left(t, x_{d_{1 t}}, \ldots, x_{d_{k t}}\right) \mid \\
& +\mid g\left(t, x_{c_{1 t}}^{m}, \ldots, x_{c_{k t}}^{m}\right) \\
& \left.-g\left(t, x_{c_{1 t}}, \ldots, x_{c_{k t}}\right) \mid\right] \\
& +\sum_{j=T+\tau}^{\infty} \sum_{s=j}^{\infty} \sum_{t=s}^{\infty} \frac{t-s+1}{\left|a_{j}\right|} \mid p\left(t, x_{o_{1 t}}^{m}, \ldots, x_{o_{k t}}^{m}\right)
\end{aligned}
$$

$$
\left.-p\left(t, x_{o_{1 t}}, \ldots, x_{o_{k t}}\right) \mid\right)
$$

$$
\begin{gathered}
\leq \frac{1}{c}\left(\sum_{t=T+\tau}^{T_{1}+\tau-1} \frac{1}{\left|a_{t}\right|}\left|f\left(t, x_{b_{1 t}}^{m}, \ldots, x_{b_{k t}}^{m}\right)-f\left(t, x_{b_{1 t}}, \ldots, x_{b_{k t}}\right)\right|\right. \\
+\sum_{t=T_{1}+\tau}^{\infty} \frac{1}{\left|a_{t}\right|} \mid f\left(t, x_{b_{1 t}}^{m}, \ldots, x_{b_{k t}}^{m}\right) \\
+\sum_{s=T+\tau} \sum_{t=s}^{T_{1}+\tau-1} \frac{1}{\left|a_{s}\right|}\left[(t-s+1) \mid h\left(t, x_{d_{1 t}}^{m}, \ldots, x_{d_{k t}}^{m}\right)\right. \\
\left.-h\left(t, x_{b_{1 t}}, \ldots, x_{b_{k t}}\right) \mid \ldots, x_{d_{k t}}\right) \mid \\
+\mid g\left(t, x_{c_{1 t}}^{m}, \ldots, x_{c_{k t}}^{m}\right) \\
\left.-g\left(t, x_{c_{1 t}}, \ldots, x_{c_{k t}}\right) \mid\right]
\end{gathered}
$$$$
+\sum_{s=T+\tau}^{T_{1}+\tau-1} \sum_{t=T_{2}+\tau}^{\infty} \frac{1}{\left|a_{s}\right|}\left[(t-s+1) \mid h\left(t, x_{d_{1 t}}^{m}, \ldots, x_{d_{k t}}^{m}\right)\right.
$$$$
-h\left(t, x_{d_{1 t}}, \ldots, x_{d_{k t}}\right)
$$$$
+\mid g\left(t, x_{c_{1 t}}^{m}, \ldots, x_{c_{k t}}^{m}\right)
$$$$
\left.-g\left(t, x_{c_{1 t}}, \ldots, x_{c_{k t}}\right) \mid\right]
$$$$
+\sum_{s=T_{1}+\tau}^{\infty} \sum_{t=s}^{\infty} \frac{1}{\left|a_{s}\right|}\left[(t-s+1) \mid h\left(t, x_{d_{1 t}}^{m}, \ldots, x_{d_{k t}}^{m}\right)\right.
$$$$
-h\left(t, x_{d_{1 t}}, \ldots, x_{d_{k t}}\right)
$$$$
+\mid g\left(t, x_{c_{1 t}}^{m}, \ldots, x_{c_{k t}}^{m}\right)
$$$$
\left.-g\left(t, x_{c_{1 t}}, \ldots, x_{c_{k t}}\right) \mid\right]
$$$$
+\sum_{j=T+\tau}^{T_{1}+\tau-1} \sum_{s=j}^{T_{2}+\tau-1} \sum_{t=s}^{T_{3}+\tau-1} \frac{t-s+1}{\left|a_{j}\right|} \mid p\left(t, x_{o_{1 t}}^{m}, \ldots, x_{o_{k t}}^{m}\right)
$$$$
-p\left(t, x_{o_{1 \mathrm{t}}}, \ldots, x_{o_{k t}}\right)
$$$$
+\sum_{j=T+\tau}^{T_{1}+\tau-1} \sum_{s=j}^{T_{2}+\tau-1} \sum_{t=T_{3}+\tau}^{\infty} \frac{t-s+1}{\left|a_{j}\right|} \mid p\left(t, x_{o_{1 t}}^{m}, \ldots, x_{o_{k t}}^{m}\right)
$$$$
-p\left(t, x_{o_{1 t}}, \ldots, x_{o_{k t}}\right)
$$

$$
+\sum_{j=T+\tau}^{T_{1}+\tau-1} \sum_{s=T_{2}+\tau}^{\infty} \sum_{t=s}^{\infty} \frac{t-s+1}{\left|a_{j}\right|} \mid p\left(t, x_{o_{1 t}}^{m}, \ldots, x_{o_{k t}}^{m}\right)
$$

$$
-p\left(t, x_{o_{1 t}}, \ldots, x_{o_{k t}}\right) \mid
$$




$$
\begin{aligned}
& +\sum_{j=T_{1}+\tau}^{\infty} \sum_{s=j}^{\infty} \sum_{t=s}^{\infty} \frac{t-s+1}{\left|a_{j}\right|} \mid p\left(t, x_{o_{1 t}}^{m}, \ldots, x_{o_{k t}}^{m}\right) \\
& +\frac{2 \varepsilon\left(T_{1}-T\right)}{18 T_{1}}+\frac{2 \varepsilon\left(T_{1}-T\right)\left(T_{2}-T\right)}{18 T_{1} T_{2}} \\
& \left.-p\left(t, x_{o_{1 t}}, \ldots, x_{o_{k t}}\right) \mid\right) \quad+\frac{2 \varepsilon\left(T_{1}-T\right)}{18 T_{1}}+\frac{\varepsilon}{18}<\varepsilon, \quad \forall m \geq T_{4} \text {, } \\
& \leq \frac{1}{c}\left(\sum_{t=T+\tau}^{T_{1}+\tau-1} A \mid f\left(t, x_{b_{1 t}}^{m}, \ldots, x_{b_{k t}}^{m}\right)\right. \\
& -f\left(t, x_{b_{1 t}}, \ldots, x_{b_{k t}}\right) \\
& +\sum_{s=T+\tau}^{T_{1}+\tau-1} \sum_{t=s}^{T_{2}+\tau-1}\left[B \mid h\left(t, x_{d_{1 t}}^{m}, \ldots, x_{d_{k t}}^{m}\right)\right. \\
& -h\left(t, x_{d_{1 t}}, \ldots, x_{d_{k t}}\right) \\
& +A \mid g\left(t, x_{c_{1 t}}^{m}, \ldots, x_{c_{k t}}^{m}\right) \\
& \left.-g\left(t, x_{c_{1 t}}, \ldots, x_{c_{k t}}\right) \mid\right] \\
& +\sum_{j=T+\tau}^{T_{1}+\tau-1} \sum_{s=j}^{T_{2}+\tau-1} \sum_{t=s}^{T_{3}+\tau-1} E \mid p\left(t, x_{o_{1 t}}^{m}, \ldots, x_{o_{k t}}^{m}\right) \\
& -p\left(t, x_{o_{1 t}}, \ldots, x_{o_{k t}}\right) \\
& +2 \sum_{t=T_{1}+\tau}^{\infty} \frac{W_{t}}{\left|a_{t}\right|} \\
& +2 \sum_{s=T+\tau}^{T_{1}+\tau-1} \sum_{t=T_{2}+\tau}^{\infty} \frac{1}{\left|a_{s}\right|}\left[(t-s+1) Q_{t}+P_{t}\right] \\
& +2 \sum_{s=T_{1}+\tau}^{\infty} \sum_{t=s}^{\infty} \frac{1}{\left|a_{s}\right|}\left[(t-s+1) Q_{t}+P_{t}\right] \\
& +2 \sum_{j=T+\tau}^{T_{1}+\tau-1} \sum_{s=j}^{T_{2}+\tau-1} \sum_{t=T_{3}+\tau}^{\infty} \frac{t-s+1}{\left|a_{j}\right|} R_{t} \\
& +2 \sum_{j=T+\tau} \sum_{s=T_{2}+\tau} \sum_{t=s}^{\infty} \frac{t-s+1}{\left|a_{j}\right|} R_{t} \\
& \left.+2 \sum_{j=T_{1}+\tau}^{\infty} \sum_{s=j}^{\infty} \sum_{t=s}^{\infty} \frac{t-s+1}{\left|a_{j}\right|} R_{t}\right) \\
& <\frac{\varepsilon A\left(T_{1}-T\right)}{18 T_{1} T_{2} T_{3}(A+B+E)} \\
& +\frac{\varepsilon(B+A)\left(T_{1}-T\right)\left(T_{2}-T\right)}{18 T_{1} T_{2} T_{3}(A+B+E)} \\
& +\frac{\varepsilon E\left(T_{1}-T\right)\left(T_{2}-T\right)\left(T_{3}-T\right)}{18 T_{1} T_{2} T_{3}(A+B+E)} \\
& \sum_{t=V+\tau}^{\infty} \frac{W_{t}}{\left|a_{t}\right|} \\
& +\sum_{s=V+\tau}^{\infty} \sum_{t=s}^{\infty} \frac{1}{\left|a_{s}\right|}\left[(t-s+1) Q_{t}+P_{t}\right] \\
& +\sum_{j=V+\tau}^{\infty} \sum_{s=j}^{\infty} \sum_{t=s}^{\infty} \frac{t-s+1}{\left|a_{j}\right|}\left(R_{t}+\left|r_{t}\right|\right)<\frac{\varepsilon}{4} . \\
& \left|\left(S_{L} x\right)_{m}-\left(S_{L} x\right)_{n}\right| \\
& =\mid \frac{1}{\gamma_{m+\tau}} \sum_{t=m+\tau}^{\infty} \frac{1}{a_{t}} f\left(t, x_{b_{1 t}}, \ldots, x_{b_{k t}}\right) \\
& -\frac{1}{\gamma_{n+\tau}} \sum_{t=n+\tau}^{\infty} \frac{1}{a_{t}} f\left(t, x_{b_{1 t}}, \ldots, x_{b_{k t}}\right) \\
& +\frac{1}{\gamma_{m+\tau}} \sum_{s=m+\tau}^{\infty} \sum_{t=s}^{\infty} \frac{1}{a_{s}}\left[(t-s+1) h\left(t, x_{d_{1 t}}, \ldots, x_{d_{k t}}\right)\right. \\
& \left.-g\left(t, x_{c_{1 t}}, \ldots, x_{c_{k t}}\right)\right] \\
& -\frac{1}{\gamma_{n+\tau}} \sum_{s=n+\tau}^{\infty} \sum_{t=s}^{\infty} \frac{1}{a_{s}}\left[(t-s+1) h\left(t, x_{d_{1 t}}, \ldots, x_{d_{k t}}\right)\right. \\
& \left.-g\left(t, x_{c_{1 t}}, \ldots, x_{c_{k t}}\right)\right] \\
& \left.+\frac{1}{\gamma_{n+\tau}} \sum_{j=n+\tau s=j}^{\infty} \sum_{j=s}^{\infty} \sum_{t=s}^{t-s+1} \frac{t}{a_{j}}\left[t, x_{o_{1 t}}, \ldots, x_{o_{k t}}\right)-r_{t}\right] \\
& -\frac{1}{\gamma_{m+\tau}} \sum_{j=m+\tau}^{\infty} \sum_{s=j}^{\infty} \sum_{t=s}^{\infty} \frac{t-s+1}{a_{j}}\left[p\left(t, x_{o_{1 t}}, \ldots, x_{o_{k t}}\right)-r_{t}\right] \mid
\end{aligned}
$$




$$
\begin{aligned}
& \leq \frac{1}{c}\left(\sum_{t=m+\tau}^{\infty} \frac{W_{t}}{\left|a_{t}\right|}+\sum_{t=n+\tau}^{\infty} \frac{W_{t}}{\left|a_{t}\right|}\right. \\
& +\sum_{s=m+\tau}^{\infty} \sum_{t=s}^{\infty} \frac{1}{\left|a_{s}\right|}\left[(t-s+1) Q_{t}+P_{t}\right] \\
& +\sum_{s=n+\tau}^{\infty} \sum_{t=s}^{\infty} \frac{1}{\left|a_{s}\right|}\left[(t-s+1) Q_{t}+P_{t}\right] \\
& +\sum_{j=n+\tau}^{\infty} \sum_{s=j}^{\infty} \sum_{t=s}^{\infty} \frac{t-s+1}{\left|a_{j}\right|}\left(R_{t}+\left|r_{t}\right|\right) \\
& \left.+\sum_{j=m+\tau}^{\infty} \sum_{s=j}^{\infty} \sum_{t=s}^{\infty} \frac{t-s+1}{\left|a_{j}\right|}\left(R_{t}+\left|r_{t}\right|\right)\right) \\
& \leq \frac{2}{c}\left(\sum_{t=V+\tau}^{\infty} \frac{W_{t}}{\left|a_{t}\right|}\right. \\
& \quad+\sum_{s=V+\tau}^{\infty} \sum_{j=s}^{\infty} \frac{1}{\left|a_{s}\right|}\left[(t-s+1) Q_{t}+P_{t}\right] \\
& \left.+\sum_{j=V+\tau}^{\infty} \sum_{s=j}^{\infty} \sum_{t=s}^{\infty} \frac{t-s+1}{\left|a_{j}\right|}\left(R_{t}+\left|r_{t}\right|\right)\right)
\end{aligned}
$$$$
<\varepsilon
$$

Note that (40) and (42) yield that

$$
\left\|S_{L} x\right\|
$$

$$
\begin{aligned}
& =\sup _{n \in \mathbb{Z}_{\beta}}\left|\left(S_{L} x\right)_{n}\right| \\
& =\max \left\{\sup _{T>n \geq \beta}\left|\left(S_{L} x\right)_{n}\right|, \sup _{n \geq T}\left|\left(S_{L} x\right)_{n}\right|\right\} \\
& =\sup _{n \geq T} \mid \frac{1}{\gamma_{n+\tau}}\left(\sum_{t=n+\tau}^{\infty} \frac{1}{a_{t}} f\left(t, x_{b_{1 t}}, \ldots, x_{b_{k t}}\right)\right. \\
& \quad+\sum_{s=n+\tau t=s}^{\infty} \sum^{\infty} \frac{1}{a_{s}}\left[(t-s+1) h\left(t, x_{d_{1 t}}, \ldots, x_{d_{k t}}\right)\right. \\
& \left.\quad-\sum_{j=n+\tau s=j t=s}^{\infty} \sum^{\infty} \frac{t-s+1}{a_{j}}\left[p\left(t, x_{o_{1 t}}, \ldots, x_{o_{k t}}\right)-r_{t}\right]\right) \mid
\end{aligned}
$$

$$
\begin{aligned}
& \leq \frac{1}{c}\left(\sum_{t=T+\tau}^{\infty} \frac{W_{t}}{\left|a_{t}\right|}\right. \\
& +\sum_{s=T+\tau}^{\infty} \sum_{t=s}^{\infty} \frac{1}{\left|a_{s}\right|}\left[(t-s+1) Q_{t}+P_{t}\right] \\
& \left.+\sum_{j=T+\tau}^{\infty} \sum_{s=j}^{\infty} \sum_{t=s}^{\infty} \frac{t-s+1}{\left|a_{j}\right|}\left(R_{t}+\left|r_{t}\right|\right)\right) \\
& <\frac{1}{c} \min \left\{M-L, L-N-\frac{M}{c}\right\} \\
& <\frac{M}{c}, \quad \forall x=\left\{x_{n}\right\}_{n \in \mathbb{Z}_{\beta}} \in \Omega_{2 T}(N, M),
\end{aligned}
$$

which gives that $S_{L}\left(\Omega_{2 T}(N, M)\right)$ is bounded. Thus Lemma 3 means that there exists $x=\left\{x_{n}\right\}_{n \in \mathbb{Z}_{\beta}} \in \Omega_{2 T}(N, M)$ such that $U_{L} x+S_{L} x=x$, which is a bounded positive solution of (2) in $\Omega_{2 T}(N, M) \subset l_{\beta}^{\infty}$.

Let $L_{1}, L_{2} \in(N+M / c, M)$ and $L_{1} \neq L_{2}$. For any $j \in$ $\{1,2\}$, we deduce similarly that there exist a positive integer $T_{j} \geq n_{0}+n_{1}+\tau+|\beta|$, a closed bounded and convex subset $\Omega_{2 T_{j}}(N, M)$ of $l_{\beta}^{\infty}$, and two mappings $U_{L_{j}}$ and $S_{L_{j}}$ satisfying (40)-(42), where $L$ and $T$ are replaced by $L_{j}$ and $T_{j}$, respectively, and $U_{L_{j}}+S_{L_{j}}$ possesses a fixed point $z_{j}=$ $\left\{z_{\text {jn }}\right\}_{n \in \mathbb{Z}_{\beta}} \in \Omega_{2 T_{j}}(N, M)$, which is a bounded positive solution of (2); that is,

$$
\begin{array}{r}
z_{j n} \\
=\frac{1}{\gamma_{n+\tau}}\left(L_{j}-z_{j n+\tau}+\sum_{t=n+\tau}^{\infty} \frac{1}{a_{t}} f\left(t, z_{j b_{1 t}}, \ldots, z_{j b_{k t}}\right)\right. \\
+\sum_{s=n+\tau}^{\infty} \sum_{t=s}^{\infty} \frac{1}{a_{s}}\left[(t-s+1) h\left(t, z_{j d_{1 t}}, \ldots, z_{j d_{k t}}\right)\right. \\
\left.-g\left(t, z_{j c_{1 t}}, \ldots, z_{j c_{k t}}\right)\right] \\
\left.-\sum_{j=n+\tau s=j t=s}^{\infty} \sum_{j}^{\infty} \frac{t-s+1}{a_{j}}\left[p\left(t, z_{j o_{1 t}}, \ldots, z_{j o_{k t}}\right)-r_{t}\right]\right), \\
\forall n \geq T_{j}, j \in\{1,2\} .
\end{array}
$$

Observe that (13) implies that there exists $T_{3} \in \mathbb{N}$ with $T_{3}>$ $\max \left\{T_{1}, T_{2}\right\}$ satisfying

$$
\begin{aligned}
& \sum_{t=T_{3}+\tau}^{\infty} \frac{W_{t}}{\left|a_{t}\right|} \\
& +\sum_{s=T_{3}+\tau}^{\infty} \sum_{t=s}^{\infty} \frac{1}{\left|a_{s}\right|}\left[(t-s+1) Q_{t}+P_{t}\right] \\
& \quad+\sum_{j=T_{3}+\tau}^{\infty} \sum_{s=j}^{\infty} \sum_{t=s}^{\infty} \frac{t-s+1}{\left|a_{j}\right|} R_{t}<\frac{\left|L_{1}-L_{2}\right|}{4},
\end{aligned}
$$


which together with (51) yields that for each $n \geq T_{3}$

$$
\begin{aligned}
& \left|z_{1 n}-z_{2 n}+\frac{z_{1 n+\tau}-z_{2 n+\tau}}{\gamma_{n+\tau}}\right| \\
& =\mid \frac{1}{\gamma_{n+\tau}}\left(L_{1}-L_{2}+\sum_{t=n+\tau}^{\infty} \frac{1}{a_{t}} f\left(t, z_{1 b_{1 t}}, \ldots, z_{1 b_{k t}}\right)\right. \\
& -\sum_{t=n+\tau}^{\infty} \frac{1}{a_{t}} f\left(t, z_{2 b_{1 t}}, \ldots, z_{2 b_{k t}}\right) \\
& +\sum_{s=n+\tau t=s}^{\infty} \sum_{s}^{\infty} \frac{1}{a_{s}}\left[(t-s+1) h\left(t, z_{1 d_{1 t}}, \ldots, z_{1 d_{k t}}\right)\right. \\
& \left.-g\left(t, z_{1 c_{1 t}}, \ldots, z_{1 c_{k t}}\right)\right] \\
& -\sum_{s=n+\tau}^{\infty} \sum_{t=s}^{\infty} \frac{1}{a_{s}}\left[(t-s+1) h\left(t, z_{2 d_{1 t}}, \ldots, z_{2 d_{k t}}\right)\right. \\
& \left.-g\left(t, z_{2 c_{1 t}}, \ldots, z_{2 c_{k t}}\right)\right] \\
& -\sum_{j=n+\tau s=j}^{\infty} \sum_{t=s}^{\infty} \frac{t-s+1}{a_{j}}\left[p\left(t, z_{1 o_{1 t}}, \ldots, z_{1 o_{k t}}\right)-r_{t}\right] \\
& \left.+\sum_{j=n+\tau s=j}^{\infty} \sum_{t=s}^{\infty} \sum_{t}^{t-s+1} \frac{t}{a_{j}}\left[p\left(t, z_{2 o_{1 t}}, \ldots, z_{2 o_{k t}}\right)-r_{t}\right]\right) \mid \\
& \geq \frac{1}{\gamma_{n+\tau}}\left(\left|L_{1}-L_{2}\right|-\sum_{t=n+\tau}^{\infty} \frac{1}{a_{t}} \mid f\left(t, z_{1 b_{1 t}}, \ldots, z_{1 b_{k t}}\right)\right. \\
& -f\left(t, z_{2 b_{1 t}}, \ldots, z_{2 b_{k t}}\right) \\
& -\sum_{s=n+\tau}^{\infty} \sum_{t=s}^{\infty} \frac{1}{a_{s}}\left[(t-s+1) \mid h\left(t, z_{1 d_{1 t}}, \ldots, z_{1 d_{k t}}\right)\right. \\
& -h\left(t, z_{2 d_{1 t}}, \ldots, z_{2 d_{k t}}\right) \\
& +\mid g\left(t, z_{1 c_{1 t}}, \ldots, z_{1 c_{k t}}\right) \\
& \left.-g\left(t, z_{2 c_{1 t}}, \ldots, z_{2 c_{k t}}\right) \mid\right] \\
& -\sum_{j=n+\tau}^{\infty} \sum_{s=j}^{\infty} \sum_{t=s}^{\infty} \frac{t-s+1}{a_{j}} \mid p\left(t, z_{1 o_{1 t}}, \ldots, z_{1 o_{k t}}\right) \\
& \left.-p\left(t, z_{2 o_{1 t}}, \ldots, z_{2 o_{k t}}\right) \mid\right) \\
& \geq \frac{1}{\gamma_{n+\tau}}\left(\left|L_{1}-L_{2}\right|-2 \sum_{t=T_{3}+\tau}^{\infty} \frac{W_{t}}{\left|a_{t}\right|}\right. \\
& -2 \sum_{s=T_{3}+\tau}^{\infty} \sum_{t=s}^{\infty} \frac{1}{\left|a_{s}\right|}\left[(t-s+1) Q_{t}+P_{t}\right] \\
& \left.-2 \sum_{j=T_{3}+\tau}^{\infty} \sum_{s=j}^{\infty} \sum_{t=s}^{\infty} \frac{t-s+1}{\left|a_{j}\right|} R_{t}\right)
\end{aligned}
$$

$$
\begin{aligned}
& \geq \frac{1}{\gamma_{n+\tau}}\left(\left|L_{1}-L_{2}\right|-\frac{\left|L_{1}-L_{2}\right|}{2}\right) \\
& >\frac{\left|L_{1}-L_{2}\right|}{2 \gamma_{n+\tau}},
\end{aligned}
$$

which implies that

$$
\left|z_{1 n}-z_{2 n}+\frac{z_{1 n+\tau}-z_{2 n+\tau}}{\gamma_{n+\tau}}\right|>0, \quad \forall n \geq T_{3}
$$

that is, $z_{1} \neq z_{2}$. Consequently, (2) has uncountably many bounded positive solutions in $l_{\beta}^{\infty}$. This completes the proof.

Theorem 10. Assume that there exist constants $n_{1} \in \mathbb{N}_{n_{0}}, M$, $N, c$, and $d$ with $d>c>1$ and $M(1-1 / c)>N(1-1 / d)$ and nonnegative sequences $\left\{W_{n}\right\}_{n \in \mathbb{N}_{n_{0}}},\left\{P_{n}\right\}_{n \in \mathbb{N}_{n_{0}}},\left\{Q_{n}\right\}_{n \in \mathbb{N}_{n_{0}}}$, and $\left\{R_{n}\right\}_{n \in \mathbb{N}_{n_{0}}}$ satisfying (13):

$$
\begin{aligned}
c \leq \gamma_{n} \leq d, \quad \forall n \geq n_{1}, \\
\left|f\left(n, u_{1}, u_{2}, \ldots, u_{k}\right)\right| \leq W_{n}, \quad\left|g\left(n, u_{1}, u_{2}, \ldots, u_{k}\right)\right| \leq P_{n}, \\
\left|h\left(n, u_{1}, u_{2}, \ldots, u_{k}\right)\right| \leq Q_{n}, \quad\left|p\left(n, u_{1}, u_{2}, \ldots, u_{k}\right)\right| \leq R_{n}, \\
\forall\left(n, u_{1}, u_{2}, \ldots, u_{k}\right) \in \mathbb{N}_{n_{0}} \times\left[\frac{N}{d}, \frac{M}{c}\right]^{k} .
\end{aligned}
$$

Then (2) possesses uncountably many bounded positive solutions in $l_{\beta}^{\infty}$.

Proof. Let $L \in(N+M / c, M+N / d)$. It follows from (13) that there exists an integer $T \geq n_{0}+n_{1}+\tau+|\beta|$ satisfying

$$
\begin{gathered}
\sum_{t=T}^{\infty} \frac{W_{t}}{\left|a_{t}\right|}+\sum_{s=T}^{\infty} \sum_{t=s}^{\infty} \frac{1}{\left|a_{s}\right|}\left[(t-s+1) Q_{t}+P_{t}\right] \\
+\sum_{j=T}^{\infty} \sum_{s=j}^{\infty} \sum_{t=s}^{\infty} \frac{t-s+1}{\left|a_{j}\right|}\left(R_{t}+\left|r_{t}\right|\right) \\
<\min \left\{M+\frac{N}{d}-L, L-N-\frac{M}{c}\right\} .
\end{gathered}
$$

Let the mappings $U_{L}$ and $S_{L}: \Omega_{2 T}(N, M) \rightarrow l_{\beta}^{\infty}$ be defined by (41) and (42), respectively. Using (13) and (41), (42) and 
(57), we obtain that for any $x=\left\{x_{n}\right\}_{n \in \mathbb{Z}_{\beta}}, y=\left\{y_{n}\right\}_{n \in \mathbb{Z}_{\beta}} \in$ $\Omega_{2 T}(N, M)$ and $n \geq T$,

$$
\begin{aligned}
& \left(U_{L} x\right)_{n}+\left(S_{L} y\right)_{n} \\
& =\frac{1}{\gamma_{n+\tau}}\left(L-x_{n+\tau}+\sum_{t=n+\tau}^{\infty} \frac{1}{a_{t}} f\left(t, y_{b_{1 t}}, \ldots, y_{b_{k t}}\right)\right. \\
& +\sum_{s=n+\tau}^{\infty} \sum_{t=s}^{\infty} \frac{1}{a_{s}}\left[(t-s+1) h\left(t, y_{d_{1 t}}, \ldots, y_{d_{k t}}\right)\right. \\
& \left.-g\left(t, y_{c_{1 t}}, \ldots, y_{c_{k t}}\right)\right] \\
& \left.-\sum_{j=n+\tau s=j}^{\infty} \sum_{t=s}^{\infty} \frac{t-s+1}{a_{j}}\left[p\left(t, y_{o_{1 t}}, \ldots, y_{o_{k t}}\right)-r_{t}\right]\right) \\
& \leq \frac{1}{\gamma_{n+\tau}}\left(L-\frac{N}{\gamma_{n+\tau}}+\sum_{t=T+\tau}^{\infty} \frac{W_{t}}{\left|a_{t}\right|}\right. \\
& +\sum_{s=T+\tau}^{\infty} \sum_{t=s}^{\infty} \frac{1}{\left|a_{s}\right|}\left[(t-s+1) Q_{t}+P_{t}\right] \\
& \left.+\sum_{j=T+\tau}^{\infty} \sum_{s=j}^{\infty} \sum_{t=s}^{\infty} \frac{t-s+1}{a_{j}}\left(R_{t}+\left|r_{t}\right|\right)\right) \\
& <\frac{1}{\gamma_{n+\tau}}\left(L-\frac{N}{d}+\min \left\{M+\frac{N}{d}-L, L-N-\frac{M}{c}\right\}\right) \\
& \leq \frac{M}{\gamma_{n+\tau}}
\end{aligned}
$$$$
\left(U_{L} x\right)_{n}+\left(S_{L} y\right)_{n}
$$$$
=\frac{1}{\gamma_{n+\tau}}\left(L-x_{n+\tau}+\sum_{t=n+\tau}^{\infty} \frac{1}{a_{t}} f\left(t, y_{b_{1 t}}, \ldots, y_{b_{k t}}\right)\right.
$$$$
+\sum_{s=n+\tau t=s}^{\infty} \sum_{a_{s}}^{\infty} \frac{1}{a_{s}}\left[(t-s+1) h\left(t, y_{d_{1 t}}, \ldots, y_{d_{k t}}\right)\right.
$$$$
\left.-g\left(t, y_{c_{1 t}}, \ldots, y_{c_{k t}}\right)\right]
$$$$
\left.-\sum_{j=n+\tau s=j t=s}^{\infty} \sum^{\infty} \sum^{\infty} \frac{t-s+1}{a_{j}}\left[p\left(t, y_{o_{1 t}}, \ldots, y_{o_{k t}}\right)-r_{t}\right]\right)
$$$$
\geq \frac{1}{\gamma_{n+\tau}}\left(L-\frac{M}{\gamma_{n+\tau}}-\sum_{t=T+\tau}^{\infty} \frac{W_{t}}{\left|a_{t}\right|}\right.
$$$$
-\sum_{s=T+\tau}^{\infty} \sum_{t=s}^{\infty} \frac{1}{\left|a_{s}\right|}\left[(t-s+1) Q_{t}+P_{t}\right]
$$$$
\left.-\sum_{j=T+\tau}^{\infty} \sum_{s=j}^{\infty} \sum_{t=s}^{\infty} \frac{t-s+1}{a_{j}}\left(R_{t}+\left|r_{t}\right|\right)\right)
$$

$$
\begin{aligned}
& >\frac{1}{\gamma_{n+\tau}}\left(L-\frac{M}{c}-\min \left\{M+\frac{N}{d}-L, L-N-\frac{M}{c}\right\}\right) \\
& \geq \frac{N}{\gamma_{n+\tau}},
\end{aligned}
$$

which imply that $U_{L} x+S_{L} y \in \Omega_{2 T}(N, M)$ for any $x, y \in$ $\Omega_{2 T}(N, M)$. The rest of the proof is similar to that of Theorem 9 and is omitted. This completes the proof.

Theorem 11. Assume that there exist constants $n_{1} \in \mathbb{N}_{n_{0}}, M$, $N$, and $c$ with $M>N>0$ and $c>M /(M-N)$ and nonnegative sequences $\left\{W_{n}\right\}_{n \in \mathbb{N}_{n_{0}}},\left\{P_{n}\right\}_{n \in \mathbb{N}_{n_{0}}},\left\{Q_{n}\right\}_{n \in \mathbb{N}_{n_{0}}}$, and $\left\{R_{n}\right\}_{n \in \mathbb{N}_{n_{0}}}$ satisfying (13), (39), and

$$
\gamma_{n} \leq-c, \quad \forall n \geq n_{1} .
$$

Then (2) possesses uncountably many bounded positive solutions in $l_{\beta}^{\infty}$.

Proof. Let $L \in(N, M(1-1 / c))$. It follows from (13) that there exists an integer $T \geq n_{0}+n_{1}+\tau+|\beta|$ satisfying

$$
\begin{aligned}
\sum_{t=T}^{\infty} \frac{W_{t}}{\left|a_{t}\right|} & +\sum_{s=T}^{\infty} \sum_{t=s}^{\infty} \frac{1}{\left|a_{s}\right|}\left[(t-s+1) Q_{t}+P_{t}\right] \\
& +\sum_{j=T}^{\infty} \sum_{s=j}^{\infty} \sum_{t=s}^{\infty} \frac{t-s+1}{\left|a_{j}\right|}\left(R_{t}+\left|r_{t}\right|\right) \\
& <\min \left\{L-N, M-\frac{M}{c}-L\right\} .
\end{aligned}
$$

Define two mappings $U_{L}$ and $S_{L}: \Omega_{3 T}(N, M) \rightarrow l_{\beta}^{\infty}$ by $(42)$ and

$$
\left(U_{L} x\right)_{n}= \begin{cases}\frac{1}{\gamma_{n+\tau}}\left(-L-x_{n+\tau}\right), & n \geq T, \\ \left(U_{L} x\right)_{T}, & \beta \leq n<T,\end{cases}
$$

for each $x=\left\{x_{n}\right\}_{n \in \mathbb{Z}_{\beta}} \in \Omega_{3 T}(N, M)$. It follows from (42) and (59)-(61) that for any $x=\left\{x_{n}\right\}_{n \in \mathbb{Z}_{\beta}}, y=\left\{y_{n}\right\}_{n \in \mathbb{Z}_{\beta}} \in$ $\Omega_{3 T}(N, M)$, and $n \geq T$,

$$
\begin{aligned}
& \left(U_{L} x\right)_{n}+\left(S_{L} y\right)_{n} \\
& =\frac{1}{\gamma_{n+\tau}}\left(-L-x_{n+\tau}+\sum_{t=n+\tau}^{\infty} \frac{1}{a_{t}} f\left(t, y_{b_{1 t}}, \ldots, y_{b_{k t}}\right)\right. \\
& +\sum_{s=n+\tau t=s}^{\infty} \sum_{a_{s}}^{\infty} \frac{1}{[}\left[(t-s+1) h\left(t, y_{d_{1 t}}, \ldots, y_{d_{k t}}\right)\right. \\
& \left.-g\left(t, y_{c_{1 t}}, \ldots, y_{c_{k t}}\right)\right] \\
& -\sum_{j=n+\tau s=j t=s}^{\infty} \sum^{\infty} \sum^{\infty} \frac{t-s+1}{a_{j}}\left[p\left(t, y_{o_{1 t}}, \ldots, y_{o_{k t}}\right)\right. \\
& \left.\left.-r_{t}\right]\right)
\end{aligned}
$$




$$
\begin{aligned}
& \leq \frac{1}{\gamma_{n+\tau}}\left(-L+\frac{M}{\gamma_{n+\tau}}-\sum_{t=T+\tau}^{\infty} \frac{W_{t}}{\left|a_{t}\right|}\right. \\
& -\sum_{s=T+\tau}^{\infty} \sum_{t=s}^{\infty} \frac{1}{\left|a_{s}\right|}\left[(t-s+1) Q_{t}+P_{t}\right] \\
& \left.-\sum_{j=T+\tau}^{\infty} \sum_{s=j}^{\infty} \sum_{t=s}^{\infty} \frac{t-s+1}{a_{j}}\left(R_{t}+\left|r_{t}\right|\right)\right) \\
& <\frac{1}{\gamma_{n+\tau}}\left(-L-\frac{M}{c}-\min \left\{L-N, M-\frac{M}{c}-L\right\}\right) \\
& \leq \frac{-M}{\gamma_{n+\tau}}, \\
& \left(U_{L} x\right)_{n}+\left(S_{L} y\right)_{n} \\
& =\frac{1}{\gamma_{n+\tau}}\left(-L-x_{n+\tau}+\sum_{t=n+\tau}^{\infty} \frac{1}{a_{t}} f\left(t, y_{b_{1 t}}, \ldots, y_{b_{k t}}\right)\right. \\
& +\sum_{s=n+\tau t=s}^{\infty} \sum_{a_{s}}^{\infty} \frac{1}{a_{s}}\left[(t-s+1) h\left(t, y_{d_{1 t}}, \ldots, y_{d_{k t}}\right)\right. \\
& \left.-g\left(t, y_{c_{1 t}}, \ldots, y_{c_{k t}}\right)\right] \\
& -\sum_{j=n+\tau s=j t=s}^{\infty} \sum^{\infty} \sum_{t=s}^{\infty} \frac{t-s+1}{a_{j}}\left[p\left(t, y_{o_{1 t}}, \ldots, y_{o_{k t}}\right)\right. \\
& \left.\left.-r_{t}\right]\right) \\
& \geq \frac{1}{\gamma_{n+\tau}}\left(-L-x_{n+\tau}+\sum_{t=T+\tau}^{\infty} \frac{W_{t}}{\left|a_{t}\right|}\right. \\
& +\sum_{s=T+\tau}^{\infty} \sum_{t=s}^{\infty} \frac{1}{\left|a_{s}\right|}\left[(t-s+1) Q_{t}+P_{t}\right] \\
& \left.+\sum_{j=T+\tau}^{\infty} \sum_{s=j}^{\infty} \sum_{t=s}^{\infty} \frac{t-s+1}{a_{j}}\left(R_{t}+\left|r_{t}\right|\right)\right) \\
& >\frac{1}{\gamma_{n+\tau}}\left(-L+\min \left\{L-N, M-\frac{M}{c}-L\right\}\right) \\
& \geq \frac{-N}{\gamma_{n+\tau}}
\end{aligned}
$$

which yield that $U_{L} x+S_{L} y \in \Omega_{3 T}(N, M)$. The rest of the proof is similar to that of Theorem 9 and hence is omitted. This completes the proof.

Theorem 12. Assume that there exist constants $n_{1} \in \mathbb{N}_{n_{0}}, M$, $N, c$, and $d$ with $M>N>0$ and $M(1-1 / c)>N(1+1 / d)$, $d>c>1$, and nonnegative sequences $\left\{W_{n}\right\}_{n \in \mathbb{N}_{n_{0}}},\left\{P_{n}\right\}_{n \in \mathbb{N}_{n_{0}}}$, $\left\{Q_{n}\right\}_{n \in \mathbb{N}_{n_{0}}}$, and $\left\{R_{n}\right\}_{n \in \mathbb{N}_{n_{0}}}$ satisfying (13), (56), and

$$
-d \leq \gamma_{n} \leq-c, \quad \forall n \geq n_{1} .
$$

Then (2) possesses uncountably many bounded positive solutions in $l_{\beta}^{\infty}$.

Proof. Let $L \in(N(1+1 / d), M(1-1 / c))$. It follows from (13) that there exists an integer $T \geq n_{0}+n_{1}+\tau+|\beta|$ satisfying

$$
\begin{aligned}
& \sum_{t=T}^{\infty} \frac{W_{t}}{\left|a_{t}\right|} \\
& +\sum_{s=T}^{\infty} \sum_{t=s}^{\infty} \frac{1}{\left|a_{s}\right|}\left[(t-s+1) Q_{t}+P_{t}\right] \\
& +\sum_{j=T}^{\infty} \sum_{s=j}^{\infty} \sum_{t=s}^{\infty} \frac{t-s+1}{\left|a_{j}\right|}\left(R_{t}+\left|r_{t}\right|\right) \\
& \quad<\min \left\{L-\frac{N}{d}-N, M-\frac{M}{c}-L\right\} .
\end{aligned}
$$

Let the mappings $U_{L}$ and $S_{L}: \Omega_{3 T}(N, M) \rightarrow l_{\beta}^{\infty}$ be defined by (42) and (61), respectively. By means of (42), (56), (61), and (64), we deduce that for any $x=\left\{x_{n}\right\}_{n \in \mathbb{Z}_{\beta}}, y=\left\{y_{n}\right\}_{n \in \mathbb{Z}_{\beta}} \in$ $\Omega_{3 T}(N, M)$, and $n \geq T$,

$$
\begin{aligned}
& \left(U_{L} x\right)_{n}+\left(S_{L} y\right)_{n} \\
& =\frac{1}{\gamma_{n+\tau}}\left(-L-x_{n+\tau}\right. \\
& +\sum_{t=n+\tau}^{\infty} \frac{1}{a_{t}} f\left(t, y_{b_{1 t}}, \ldots, y_{b_{k t}}\right) \\
& +\sum_{s=n+\tau t=s}^{\infty} \sum_{a_{s}}^{\infty} \frac{1}{a_{s}}\left[(t-s+1) h\left(t, y_{d_{1 t}}, \ldots, y_{d_{k t}}\right)\right. \\
& \left.-g\left(t, y_{c_{1 t}}, \ldots, y_{c_{k t}}\right)\right] \\
& \left.-\sum_{j=n+\tau s=j t=s}^{\infty} \sum^{\infty} \sum^{\infty} \frac{t-s+1}{a_{j}}\left[p\left(t, y_{o_{1 t}}, \ldots, y_{o_{k t}}\right)-r_{t}\right]\right) \\
& \leq \frac{1}{\gamma_{n+\tau}}\left(-L+\frac{M}{\gamma_{n+\tau}}-\sum_{t=T+\tau}^{\infty} \frac{W_{t}}{\left|a_{t}\right|}\right. \\
& -\sum_{s=T+\tau}^{\infty} \sum_{t=s}^{\infty} \frac{1}{\left|a_{s}\right|}\left[(t-s+1) Q_{t}+P_{t}\right] \\
& \left.-\sum_{j=T+\tau s=j t=s}^{\infty} \sum^{\infty} \sum_{t=s}^{t-s+1} a_{j}\left(R_{t}+\left|r_{t}\right|\right)\right) \\
& <\frac{1}{\gamma_{n+\tau}}\left(-L-\frac{M}{c}-\min \left\{L-\frac{N}{d}-N, M-\frac{M}{c}-L\right\}\right) \\
& \leq \frac{-M}{\gamma_{n+\tau}},
\end{aligned}
$$




$$
\begin{aligned}
& \left(U_{L} x\right)_{n}+\left(S_{L} y\right)_{n} \\
& =\frac{1}{\gamma_{n+\tau}}\left(-L-x_{n+\tau}+\sum_{t=n+\tau}^{\infty} \frac{1}{a_{t}} f\left(t, y_{b_{1 t}}, \ldots, y_{b_{k t}}\right)\right. \\
& +\sum_{s=n+\tau}^{\infty} \sum_{t=s}^{\infty} \frac{1}{a_{s}}\left[(t-s+1) h\left(t, y_{d_{1 t}}, \ldots, y_{d_{k t}}\right)\right. \\
& \left.-g\left(t, y_{c_{1 t}}, \ldots, y_{c_{k t}}\right)\right] \\
& \left.-\sum_{j=n+\tau}^{\infty} \sum_{s=j t=s}^{\infty} \sum^{\infty} \frac{t-s+1}{a_{j}}\left[p\left(t, y_{o_{1 t}}, \ldots, y_{o_{k t}}\right)-r_{t}\right]\right) \\
& \geq \frac{1}{\gamma_{n+\tau}}\left(-L+\frac{N}{d}-x_{n+\tau}+\sum_{t=T+\tau}^{\infty} \frac{W_{t}}{\left|a_{t}\right|}\right. \\
& +\sum_{s=T+\tau}^{\infty} \sum_{t=s}^{\infty} \frac{1}{\left|a_{s}\right|}\left[(t-s+1) Q_{t}+P_{t}\right] \\
& \left.+\sum_{j=T+\tau}^{\infty} \sum_{s=j}^{\infty} \sum_{t=s}^{\infty} \frac{t-s+1}{a_{j}}\left(R_{t}+\left|r_{t}\right|\right)\right) \\
& >\frac{1}{\gamma_{n+\tau}}\left(-L+\frac{N}{d}+\min \left\{L-\frac{N}{d}-N, M-\frac{M}{c}-L\right\}\right) \\
& \geq \frac{-N}{\gamma_{n+\tau}},
\end{aligned}
$$

which mean that $U_{L} x+S_{L} y \in \Omega_{3 T}(N, M)$. The rest of the proof is similar to that of Theorem 9 and hence is omitted. This completes the proof.

Theorem 13. Assume that there exist constants $n_{1} \in \mathbb{N}_{n_{0}}, M$, and $N$ with $M>N>0$ and nonnegative sequences $\left\{W_{n}\right\}_{n \in \mathbb{N}_{n_{0}}}$, $\left\{P_{n}\right\}_{n \in \mathbb{N}_{n_{0}}},\left\{Q_{n}\right\}_{n \in \mathbb{N}_{n_{0}}}$, and $\left\{R_{n}\right\}_{n \in \mathbb{N}_{n_{0}}}$ satisfying (12), (13), and

$$
\gamma_{n}=1, \quad \forall n \geq n_{1}
$$

Then (2) possesses uncountably many bounded positive solutions in $\Omega_{1}(N, M)$

Proof. Let $L \in(N, M)$. Equation (13) ensures that there exists $T \geq n_{0}+n_{1}+\tau+|\beta|$ sufficiently large such that

$$
\begin{gathered}
\sum_{t=T}^{\infty} \frac{W_{t}}{\left|a_{t}\right|}+\sum_{s=T}^{\infty} \sum_{t=s}^{\infty} \frac{1}{\left|a_{s}\right|}\left[(t-s+1) Q_{t}+P_{t}\right] \\
+\sum_{j=T}^{\infty} \sum_{s=j}^{\infty} \sum_{t=s}^{\infty} \frac{t-s+1}{\left|a_{j}\right|}\left(R_{t}+\left|r_{t}\right|\right) \\
<\min \{M-L, L-N\} .
\end{gathered}
$$

Define a mapping $S_{L}: \Omega_{1}(N, M) \rightarrow l_{\beta}^{\infty}$ by

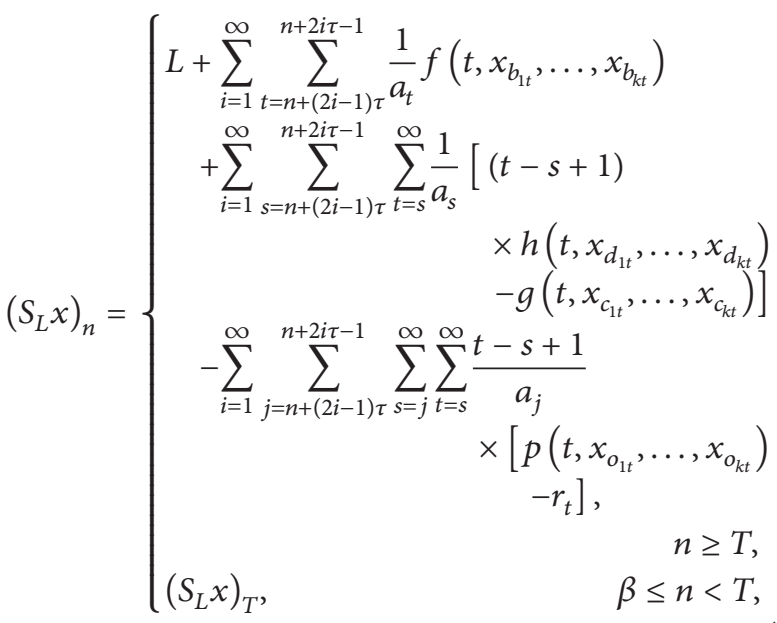

for each $x=\left\{x_{n}\right\}_{n \in \mathbb{Z}_{\beta}} \in \Omega_{1}(N, M)$. In view of (12), (67), and (68), we deduce that for every $x=\left\{x_{n}\right\}_{n \in \mathbb{Z}_{\beta}} \in \Omega_{1}(N, M)$ and $n \geq T$,

$$
\begin{aligned}
& \left|\left(S_{L} x\right)_{n}-L\right| \\
& =\mid \sum_{i=1}^{\infty} \sum_{t=n+(2 i-1) \tau}^{n+2 i \tau-1} \frac{1}{a_{t}} f\left(t, x_{b_{1 t}}, \ldots, x_{b_{k t}}\right) \\
& +\sum_{i=1}^{\infty} \sum_{s=n+(2 i-1) \tau}^{n+2 i \tau-1} \sum_{t=s}^{\infty} \frac{1}{a_{s}}\left[(t-s+1) h\left(t, x_{d_{1 t}}, \ldots, x_{d_{k t}}\right)\right. \\
& \left.-g\left(t, x_{c_{1 t}}, \ldots, x_{c_{k t}}\right)\right] \\
& -\sum_{i=1}^{\infty} \sum_{j=n+(2 i-1) \tau}^{n+2 i \tau-1} \sum_{s=j}^{\infty} \sum_{t=s}^{\infty} \frac{t-s+1}{a_{j}} \\
& \times\left[p\left(t, x_{o_{1 t}}, \ldots, x_{o_{k t}}\right)-r_{t}\right] \\
& \leq \sum_{i=1}^{\infty} \sum_{t=n+(2 i-1) \tau}^{n+2 i \tau-1} \frac{W_{t}}{\left|a_{t}\right|} \\
& +\sum_{i=1}^{\infty} \sum_{s=n+(2 i-1) \tau}^{n+2 i \tau-1} \sum_{t=s}^{\infty} \frac{1}{\left|a_{s}\right|}\left[(\mathrm{t}-s+1) Q_{t}+P_{t}\right] \\
& +\sum_{i=1}^{\infty} \sum_{j=n+(2 i-1) \tau}^{n+2 i \tau-1} \sum_{s=j}^{\infty} \sum_{t=s}^{\infty} \frac{t-s+1}{\left|a_{j}\right|}\left(R_{t}+\left|r_{t}\right|\right) \\
& \leq \sum_{t=T}^{\infty} \frac{W_{t}}{\left|a_{t}\right|}+\sum_{s=T}^{\infty} \sum_{t=s}^{\infty} \frac{1}{\left|a_{s}\right|}\left[(t-s+1) Q_{t}+P_{t}\right] \\
& +\sum_{j=T}^{\infty} \sum_{s=j}^{\infty} \sum_{t=s}^{\infty} \frac{t-s+1}{\left|a_{j}\right|}\left(R_{t}+\left|r_{t}\right|\right) \\
& <\min \{M-L, L-N\} \text {, }
\end{aligned}
$$


which means that $S_{L}\left(\Omega_{1}(N, M)\right) \subseteq \Omega_{1}(N, M)$ and $\left\|S_{L} x\right\| \leq$ $M$ for all $x \in \Omega_{1}(N, M)$. It follows from (13) that for each $\varepsilon>0$, there exists $V>T$ satisfying (22). Using (22) and (68), we obtain that for any $x=\left\{x_{n}\right\}_{n \in \mathbb{Z}_{\beta}} \in \Omega_{1}(N, M)$ and $m, n>V$,

$$
\begin{aligned}
& \left|\left(S_{L} x\right)_{m}-\left(S_{L} x\right)_{n}\right| \\
& =\mid \sum_{i=1}^{\infty} \sum_{t=m+(2 i-1) \tau}^{m+2 i \tau-1} \frac{1}{a_{t}} f\left(t, x_{b_{1 t}}, \ldots, x_{b_{k t}}\right) \\
& -\sum_{i=1}^{\infty} \sum_{t=n+(2 i-1) \tau}^{n+2 i \tau-1} \frac{1}{a_{t}} f\left(t, x_{b_{1 t}}, \ldots, x_{b_{k t}}\right) \\
& +\sum_{i=1}^{\infty} \sum_{s=m+(2 i-1) \tau}^{m+2 i \tau-1} \sum_{t=s}^{\infty} \frac{1}{a_{s}}\left[(t-s+1) h\left(t, x_{d_{1 t}}, \ldots, x_{d_{k t}}\right)\right. \\
& \left.-g\left(t, x_{c_{1 t}}, \ldots, x_{c_{k t}}\right)\right] \\
& -\sum_{i=1}^{\infty} \sum_{s=n+(2 i-1) \tau}^{n+2 i \tau-1} \sum_{t=s}^{\infty} \frac{1}{a_{s}}\left[(t-s+1) h\left(t, x_{d_{1 t}}, \ldots, x_{d_{k t}}\right)\right. \\
& \left.-g\left(t, x_{c_{1 t}}, \ldots, x_{c_{k t}}\right)\right] \\
& +\sum_{i=1}^{\infty} \sum_{j=n+(2 i-1) \tau}^{n+2 i \tau-1} \sum_{s=j}^{\infty} \sum_{t=s}^{\infty} \frac{t-s+1}{a_{j}} \\
& \times\left[p\left(t, x_{o_{1 t}}, \ldots, x_{o_{k t}}\right)-r_{t}\right] \\
& -\sum_{i=1}^{\infty} \sum_{j=m+(2 i-1) \tau}^{m+2 i \tau-1} \sum_{s=j}^{\infty} \sum_{t=s}^{\infty} \frac{t-s+1}{a_{j}} \\
& \times\left[p\left(t, x_{o_{1 t}}, \ldots, x_{o_{k t}}\right)-r_{t}\right] \\
& \leq \sum_{i=1}^{\infty} \sum_{t=m+(2 i-1) \tau}^{m+2 i \tau-1} \frac{\mathrm{W}_{t}}{\left|a_{t}\right|}+\sum_{i=1}^{\infty} \sum_{t=n+(2 i-1) \tau}^{n+2 i \tau-1} \frac{W_{t}}{\left|a_{t}\right|} \\
& +\sum_{i=1}^{\infty} \sum_{s=m+(2 i-1) \tau}^{m+2 i \tau-1} \sum_{t=s}^{\infty} \frac{1}{\left|a_{s}\right|}\left[(t-s+1) Q_{t}+P_{t}\right] \\
& +\sum_{i=1}^{\infty} \sum_{s=n+(2 i-1) \tau}^{n+2 i \tau-1} \sum_{t=s}^{\infty} \frac{1}{\left|a_{s}\right|}\left[(t-s+1) Q_{t}+P_{t}\right] \\
& +\sum_{i=1}^{\infty} \sum_{j=n+(2 i-1) \tau}^{n+2 i \tau-1} \sum_{s=j}^{\infty} \sum_{t=s}^{\infty} \frac{t-s+1}{\left|a_{j}\right|}\left(R_{t}+\left|r_{t}\right|\right) \\
& +\sum_{i=1}^{\infty} \sum_{j=m+(2 i-1) \tau}^{m+2 i \tau-1} \sum_{s=j}^{\infty} \sum_{t=s}^{\infty} \frac{t-s+1}{\left|a_{j}\right|}\left(R_{t}+\left|r_{t}\right|\right)
\end{aligned}
$$

$$
\begin{aligned}
& \leq 2 \sum_{t=V}^{\infty} \frac{W_{t}}{\left|a_{t}\right|}+2 \sum_{s=V}^{\infty} \sum_{t=s}^{\infty} \frac{1}{\left|a_{s}\right|}\left[(t-s+1) Q_{t}+P_{t}\right] \\
& \quad+2 \sum_{j=V}^{\infty} \sum_{s=j}^{\infty} \sum_{t=s}^{\infty} \frac{t-s+1}{\left|a_{j}\right|}\left(R_{t}+\left|r_{t}\right|\right) \\
& <\varepsilon,
\end{aligned}
$$

which yields that $S_{L}\left(\Omega_{1}(N, M)\right)$ is uniformly Cauchy.

Now we prove that $S_{L}$ is continuous in $\Omega_{1}(N, M)$. Suppose that $\left\{x^{m}\right\}_{m \in N}$ is an arbitrary sequence in $\Omega_{1}(N, M)$ and $x \in \Omega_{1}(N, M)$ with $\lim _{m \rightarrow \infty} x^{m}=x$, where $x^{m}=$ $\left\{x_{n}^{m}\right\}_{n \in Z_{\beta}}$ for each $m \in N$ and $x=\left\{x_{n}\right\}_{n \in \mathbb{Z}_{\beta}}$. Using (12), (13), $\lim _{m \rightarrow \infty} x^{m}=x$, and the continuity of $f, g, h$, and $p$, we conclude that for given $\varepsilon>0$, there exist $T_{1}, T_{2}, T_{3}$, and $T_{4} \in \mathbb{N}$ with $T_{4}>T_{3}>T_{2}>T_{1}>T$ satisfying (19) and (20). It follows from (19), (20), and (68) that

$$
\begin{aligned}
& \left\|S_{L} x^{m}-S_{L} x\right\| \\
& =\sup _{n \in \mathbb{Z}_{\beta}}\left|\left(S_{L} x^{m}\right)_{n}-\left(S_{L} x\right)_{n}\right| \\
& =\max \left\{\sup _{T>n \geq \beta}\left|\left(S_{L} x^{m}\right)_{n}-\left(S_{L} x\right)_{n}\right|,\right. \\
& \left.\sup _{n \geq T}\left|\left(S_{L} x^{m}\right)_{n}-\left(S_{L} x\right)_{n}\right|\right\} \\
& =\sup _{n \geq T} \mid L+\sum_{i=1}^{\infty} \sum_{t=n+(2 i-1) \tau}^{n+2 i \tau-1} \frac{1}{a_{t}} f\left(t, x_{b_{1 t}}^{m}, \ldots, x_{b_{k t}}^{m}\right) \\
& +\sum_{i=1}^{\infty} \sum_{s=n+(2 i-1) \tau}^{n+2 i \tau-1} \sum_{t=s}^{\infty} \frac{1}{a_{s}}\left[(t-s+1) h\left(t, x_{d_{1 t}}^{m}, \ldots, x_{d_{k t}}^{m}\right)\right. \\
& \left.-g\left(t, x_{c_{1 t}}^{m}, \ldots, x_{c_{k t}}^{m}\right)\right] \\
& -\sum_{i=1}^{\infty} \sum_{j=n+(2 i-1) \tau}^{n+2 i \tau-1} \sum_{s=j}^{\infty} \sum_{t=s}^{\infty} \frac{t-s+1}{a_{j}}\left[p\left(t, x_{o_{1 t}}^{m}, \ldots, x_{o_{k t}}^{m}\right)-r_{t}\right] \\
& -\left(L+\sum_{i=1}^{\infty} \sum_{t=n+(2 i-1) \tau}^{n+2 i \tau-1} \frac{1}{a_{t}} f\left(t, x_{b_{1 t}}, \ldots, x_{b_{k t}}\right)\right. \\
& +\sum_{i=1}^{\infty} \sum_{s=n+(2 i-1) \tau}^{n+2 i \tau-1} \sum_{t=s}^{\infty} \frac{1}{a_{s}}[(t-s+1) \\
& \times h\left(t, x_{d_{1 t}}, \ldots, x_{d_{k t}}\right) \\
& \left.-g\left(t, x_{c_{1 t}}, \ldots, x_{c_{k t}}\right)\right] \\
& -\sum_{i=1}^{\infty} \sum_{j=n+(2 i-1) \tau}^{n+2 i \tau-1} \sum_{s=j}^{\infty} \sum_{t=s}^{\infty} \frac{t-s+1}{a_{j}}
\end{aligned}
$$




$$
\begin{aligned}
& \left.\times\left[p\left(t, x_{o_{1 t}}, \ldots, x_{o_{k t}}\right)-r_{t}\right]\right) \mid \\
& \leq \sum_{i=1}^{\infty} \sum_{t=n+(2 i-1) \tau}^{n+2 i \tau-1} \frac{1}{\left|a_{t}\right|} \mid f\left(t, x_{b_{1 t}}^{m}, \ldots, x_{b_{k t}}^{m}\right) \\
& -f\left(t, x_{b_{1 t}}, \ldots, x_{b_{k t}}\right) \\
& +\sum_{i=1}^{\infty} \sum_{s=n+(2 i-1) \tau}^{n+2 i \tau-1} \sum_{t=s}^{\infty} \frac{1}{\left|a_{s}\right|}\left[(t-s+1) \mid h\left(t, x_{d_{1 t}}^{m}, \ldots, x_{d_{k t}}^{m}\right)\right. \\
& -h\left(t, x_{d_{1 t}}, \ldots, x_{d_{k t}}\right) \\
& +\mid g\left(t, x_{c_{1 t}}^{m}, \ldots, x_{c_{k t}}^{x}\right) \\
& \left.-g\left(t, x_{c_{1 t}}, \ldots, x_{c_{k t}}\right) \mid\right] \\
& +\sum_{i=1}^{\infty} \sum_{j=n+(2 i-1) \tau}^{n+2 i \tau-1} \sum_{s=j}^{\infty} \sum_{t=s}^{\infty} \frac{t-s+1}{\left|a_{j}\right|} \\
& \times \mid p\left(t, x_{o_{1 t}}^{m}, \ldots, x_{o_{k t}}^{m}\right) \\
& -p\left(t, x_{o_{1 t}}, \ldots, x_{o_{k t}}\right) \mid \\
& \leq \sum_{t=T}^{\infty} \frac{1}{\left|a_{t}\right|}\left|f\left(t, x_{b_{1 t}}^{m}, \ldots, x_{b_{k t}}^{m}\right)-f\left(t, x_{b_{1 t}}, \ldots, x_{b_{k t}}\right)\right| \\
& +\sum_{s=T}^{\infty} \sum_{t=s}^{\infty} \frac{1}{\left|a_{s}\right|}\left[(t-s+1) \mid h\left(t, x_{d_{1 t}}^{m}, \ldots, x_{d_{k t}}^{m}\right)\right. \\
& -h\left(t, x_{d_{1 t}}, \ldots, x_{d_{k t}}\right) \\
& x_{n-\tau}=L+\sum_{i=1}^{\infty} \sum_{t=n+(2 i-2) \tau}^{n+(2 i-1) \tau-1} \frac{1}{a_{t}} f\left(t, x_{b_{1 t}}, \ldots, x_{b_{k t}}\right) \\
& +\sum_{i=1}^{\infty} \sum_{s=n+(2 i-2) \tau}^{n+(2 i-1) \tau-1} \sum_{t=s}^{\infty} \frac{1}{a_{s}}[(t-s+1) \\
& -\sum_{i=1}^{\infty} \sum_{j=n+(2 i-1) \tau s}^{n+2 i \tau-1} \sum_{\tau=j t=s}^{\infty} \sum^{\infty} \frac{t-s+1}{a_{j}} \\
& \times\left[p\left(t, x_{o_{1 t}}, \ldots, x_{o_{k t}}\right)-r_{t}\right], \\
& \forall n \geq T+\tau, \\
& +\sum_{i=1}^{\infty} \sum_{s=n+(2 i-1) \tau}^{n+2 i \tau-1} \sum_{t=s}^{\infty} \frac{1}{a_{s}}[(t-s+1) \\
& \times h\left(t, x_{d_{1 t}}, \ldots, x_{d_{k t}}\right) \\
& \left.-g\left(t, x_{c_{1 t}}, \ldots, x_{c_{k t}}\right)\right] \\
& \times h\left(t, x_{d_{1 t}}, \ldots, x_{d_{k t}}\right) \\
& \left.-g\left(t, x_{c_{1 t}}, \ldots, x_{c_{k t}}\right)\right] \\
& -\sum_{i=1}^{\infty} \sum_{j=n+(2 i-2) \tau}^{n+(2 i-1) \tau-1} \sum_{s=j}^{\infty} \sum_{t=s}^{\infty} \frac{t-s+1}{a_{j}} \\
& \times\left[p\left(t, x_{o_{1 t}}, \ldots, x_{o_{k t}}\right)-r_{t}\right], \\
& \forall n \geq T+\tau,
\end{aligned}
$$$$
\left.+\left|g\left(t, x_{c_{1 t}}^{m}, \ldots, x_{c_{k t}}^{x}\right)-g\left(t, x_{c_{1 t}}, \ldots, x_{c_{k t}}\right)\right|\right] \quad \text { which imply that }
$$$$
+\sum_{j=T}^{\infty} \sum_{s=j}^{\infty} \sum_{t=s}^{\infty} \frac{t-s+1}{\left|a_{j}\right|} \mid p\left(t, x_{o_{1 t}}^{m}, \ldots, x_{o_{k t}}^{m}\right)
$$$$
-p\left(t, x_{o_{1 t}}, \ldots, x_{o_{k t}}\right)
$$

which imply that

$$
\begin{aligned}
& x_{n}+x_{n-\tau} \\
&=2 L+\sum_{t=n}^{\infty} \frac{1}{a_{t}} f\left(t, x_{b_{1 t}}, \ldots, x_{b_{k t}}\right) \\
&+\sum_{s=n}^{\infty} \sum_{t=s}^{\infty} \frac{1}{a_{s}}\left[(t-s+1) h\left(t, x_{d_{1 t}}, \ldots, x_{d_{k t}}\right)\right. \\
&\left.-g\left(t, x_{c_{1 t}}, \ldots, \mathrm{x}_{c_{k t}}\right)\right]
\end{aligned}
$$

which implies that $S_{L}$ is continuous in $\Omega_{1}(N, M)$. Thus Lemma 4 means that $S_{L}$ possesses a fixed point $x=\left\{x_{n}\right\}_{n \in \mathbb{Z}_{\beta}} \in$ $\Omega_{1}(N, M)$; that is,

$$
x_{n}=L+\sum_{i=1}^{\infty} \sum_{t=n+(2 i-1) \tau}^{n+2 i \tau-1} \frac{1}{a_{t}} f\left(t, x_{b_{1 t}}, \ldots, x_{b_{k t}}\right)
$$


which means that

$$
\begin{aligned}
& \Delta\left(a_{n} \Delta\left(x_{n}+x_{n-\tau}\right)\right)+\Delta f\left(n, x_{b_{1 n}}, \ldots, x_{b_{k n}}\right) \\
& +g\left(n, x_{c_{1 n}}, \ldots, x_{c_{k n}}\right) \\
& =\sum_{t=n}^{\infty} h\left(n, x_{d_{1 n}}, \ldots, x_{d_{k n}}\right) \\
& \quad-\sum_{t=n}^{\infty}(t-n+1)\left[p\left(t, x_{o_{1 t}}, \ldots, x_{o_{k t}}\right)-r_{t}\right], \\
& \quad \Delta^{3}\left(a_{n} \Delta\left(x_{n}+x_{n-\tau}\right)\right)+\Delta^{3} f\left(n, x_{b_{1 n}}, \ldots, x_{b_{k n}}\right) \\
& \quad+\Delta^{2} g\left(n, x_{c_{1 n}}, \ldots, x_{c_{k n}}\right)+\Delta h\left(n, x_{d_{1 n}}, \ldots, x_{d_{k n}}\right) \\
& \quad+p\left(n, x_{o_{1 n}}, \ldots, x_{o_{k n}}\right)=r_{n}, \quad \forall n \geq T+\tau,
\end{aligned}
$$

which yields that $x=\left\{x_{n}\right\}_{n \in \mathbb{Z}_{\beta}} \in \Omega_{1}(N, M)$ is bounded positive solution of (2). The rest of the proof is similar to that of Theorem 6 and is omitted. This completes the proof.

Theorem 14. Assume that there exist constants $n_{1} \in \mathbb{N}_{n_{0}}$, $M$, and $N$ with $M>N>0$ and nonnegative sequences $\left\{W_{n}\right\}_{n \in \mathbb{N}_{n_{0}}},\left\{P_{n}\right\}_{n \in \mathbb{N}_{n_{0}}},\left\{Q_{n}\right\}_{n \in \mathbb{N}_{n_{0}}}$, and $\left\{R_{n}\right\}_{n \in \mathbb{N}_{n_{0}}}$ satisfying (12) and

$$
\begin{gathered}
\gamma_{n}=-1, \quad \forall n \geq n_{1} ; \\
\max \left\{\sum_{i=1}^{\infty} \sum_{t=n_{0}+i \tau}^{\infty} \frac{W_{t}}{\left|a_{t}\right|},\right. \\
\sum_{i=1}^{\infty} \sum_{s=n_{0}+i \tau}^{\infty} \sum_{t=s}^{\infty} \frac{1}{\left|a_{s}\right|}\left[P_{t}+(t-s+1) Q_{t}\right], \\
\sum_{i=1}^{\infty} \sum_{j=n_{0}+i \tau}^{\infty} \sum_{s=j}^{\infty} \sum_{t=s}^{\infty} \frac{t-s+1}{\left|a_{j}\right|} \\
\left.\times\left(R_{t}+\left|r_{t}\right|\right)\right\}<+\infty .
\end{gathered}
$$

Then (2) possesses uncountably many bounded positive solution in $\Omega_{1}(N, M)$.
Proof. Let $L \in(N, M)$. It follows from (76) that there exists $T \geq n_{0}+n_{1}+\tau+|\beta|$ sufficiently large such that

$$
\begin{aligned}
& \sum_{i=1}^{\infty} \sum_{t=T+i \tau}^{\infty} \frac{W_{t}}{\left|a_{t}\right|} \\
& +\sum_{i=1}^{\infty} \sum_{s=T+i \tau}^{\infty} \sum_{t=s}^{\infty} \frac{1}{\left|a_{s}\right|}\left[(t-s+1) Q_{t}+P_{t}\right] \\
& +\sum_{i=1}^{\infty} \sum_{j=T+i \tau}^{\infty} \sum_{s=j}^{\infty} \sum_{t=s}^{\infty} \frac{t-s+1}{\left|a_{j}\right|}\left(R_{t}+\left|r_{t}\right|\right) \\
& \quad<\min \{M-L, L-N\} .
\end{aligned}
$$

Define a mapping $S_{L}: \Omega_{1}(N, M) \rightarrow l_{\beta}^{\infty}$ by

$$
\begin{aligned}
& \left(S_{L} x\right)_{n} \\
& =\left\{\begin{array}{c}
L-\sum_{i=1}^{\infty} \sum_{t=n+i \tau}^{\infty} \frac{1}{a_{t}} f\left(t, x_{b_{1 t}}, \ldots, x_{b_{k t}}\right) \\
-\sum_{i=1}^{\infty} \sum_{s=n+i \tau}^{\infty} \sum_{t=s}^{\infty} \frac{1}{a_{s}} \\
\times\left[(t-s+1) h\left(t, x_{d_{1 t}}, \ldots, x_{d_{k t}}\right)\right. \\
\left.-g\left(t, x_{c_{1 t}}, \ldots, x_{c_{k t}}\right)\right] \\
-\sum_{i=1}^{\infty} \sum_{j=n+i \tau}^{\infty} \sum_{s=j}^{\infty} \sum_{t=s}^{\infty} \frac{t-s+1}{a_{j}} \times\left[p\left(t, x_{o_{1 t}}, \ldots, x_{o_{k t}}\right)-r_{t}\right], \\
n \geq T, \\
\quad \times \leq n<T,
\end{array}\right.
\end{aligned}
$$

for all $x=\left\{x_{n}\right\}_{n \in \mathbb{Z}_{\beta}} \in \Omega_{1}(N, M)$. By virtue of (12), (77), and (78), we know that for every $x=\left\{x_{n}\right\}_{n \in \mathbb{Z}_{\beta}} \in \Omega_{1}(N, M)$ and $n \geq T$,

$$
\begin{aligned}
\left|\left(S_{L} x\right)_{n}-L\right| & |-| \sum_{i=1}^{\infty} \sum_{t=n+i \tau}^{\infty} \frac{1}{a_{t}} f\left(t, x_{b_{1 t}}, \ldots, x_{b_{k t}}\right) \\
& -\sum_{i=1}^{\infty} \sum_{s=n+i \tau}^{\infty} \sum_{t=s}^{\infty} \frac{1}{a_{s}}\left[(t-s+1) h\left(t, x_{d_{1 t}}, \ldots, x_{d_{k t}}\right)\right. \\
& -\sum_{i=1}^{\infty} \sum_{j=n+i \tau}^{\infty} \sum_{s=j}^{\infty} \frac{\sum_{t=s}^{\infty} \frac{t-s+1}{a_{j}}}{\leq} \\
\leq & \left.\left.\sum_{i=1}^{\infty} \sum_{t=n+i \tau}^{\infty} \frac{W_{t}}{\left|a_{t}\right|} \times, x_{c_{k t}}\right)\right] \\
& +\sum_{i=1}^{\infty} \sum_{s=n+i \tau}^{\infty} \sum_{t=s}^{\infty} \frac{1}{\left|a_{s}\right|}\left[(t-s+1) Q_{t}+P_{t}\right]
\end{aligned}
$$




$$
\begin{aligned}
& +\sum_{i=1}^{\infty} \sum_{j=n+i \tau}^{\infty} \sum_{s=j}^{\infty} \sum_{t=s}^{\infty} \frac{t-s+1}{\left|a_{j}\right|}\left(R_{t}+\left|r_{t}\right|\right) \\
\leq & \sum_{i=1}^{\infty} \sum_{t=T+i \tau}^{\infty} \frac{W_{t}}{\left|a_{t}\right|} \\
& +\sum_{i=1}^{\infty} \sum_{s=T+i \tau}^{\infty} \sum_{t=s}^{\infty} \frac{1}{\left|a_{s}\right|}\left[(t-s+1) Q_{t}+P_{t}\right] \\
& +\sum_{i=1}^{\infty} \sum_{j=T+i \tau}^{\infty} \sum_{s=j}^{\infty} \sum_{t=s}^{\infty} \frac{t-s+1}{\left|a_{j}\right|}\left(R_{t}+\left|r_{t}\right|\right) \\
< & \min \{M-L, L-N\},
\end{aligned}
$$

which means that $S_{L}\left(\Omega_{1}(N, M)\right) \subseteq \Omega_{1}(N, M)$ and $\left\|S_{L} x\right\| \leq$ $M$ for each $x \in \Omega_{1}(N, M)$.

Next we prove that $S_{L}\left(\Omega_{1}(N, M)\right)$ is uniformly Cauchy. It follows from (76) that for any given $\varepsilon>0$ there exists $T^{*} \geq T$ with

$$
\begin{aligned}
& \sum_{i=1}^{\infty} \sum_{t=T^{*}+i \tau}^{\infty} \frac{W_{t}}{\left|a_{t}\right|} \\
& +\sum_{i=1}^{\infty} \sum_{s=T^{*}+i \tau}^{\infty} \sum_{t=s}^{\infty} \frac{1}{\left|a_{s}\right|}\left[(t-s+1) Q_{t}+P_{t}\right] \\
& +\sum_{i=1}^{\infty} \sum_{j=T^{*}+i \tau}^{\infty} \sum_{s=j}^{\infty} \sum_{t=s}^{\infty} \frac{t-s+1}{\left|a_{j}\right|}\left(R_{t}+\left|r_{t}\right|\right) \\
& \quad<\frac{\varepsilon}{2} .
\end{aligned}
$$

It follows from (12), (78), and (80) that for any $x=\left\{x_{n}\right\}_{n \in \mathbb{Z}_{\beta}} \in$ $\Omega_{1}(N, M)$ and $m, n \geq T^{*}$,

$$
\begin{aligned}
& \left|\left(S_{L} x\right)_{m}-\left(S_{L} x\right)_{n}\right| \\
& =\mid \sum_{i=1}^{\infty} \sum_{t=n+i \tau}^{\infty} \frac{1}{a_{t}} f\left(t, x_{b_{1 t}}, \ldots, x_{b_{k t}}\right) \\
& \quad-\sum_{i=1}^{\infty} \sum_{t=m+i \tau}^{\infty} \frac{1}{a_{t}} f\left(t, x_{b_{1 t}}, \ldots, x_{b_{k t}}\right) \\
& \quad+\sum_{i=1}^{\infty} \sum_{s=n+i \tau}^{\infty} \sum_{t=s}^{\infty} \frac{1}{a_{s}}\left[(t-s+1) h\left(t, x_{d_{1 t}}, \ldots, x_{d_{k t}}\right)\right. \\
& \quad-\sum_{i=1}^{\infty} \sum_{s=m+i \tau}^{\infty} \sum_{t=s}^{\infty} \frac{1}{a_{s}}\left[(t-s+1) h\left(t, x_{d_{1 t}}, \ldots, x_{d_{k t}}\right)\right. \\
& \left.-g\left(t, x_{c_{1 t}}, \ldots, x_{c_{k t}}\right)\right]
\end{aligned}
$$

$$
\begin{aligned}
& +\sum_{i=1}^{\infty} \sum_{j=n+i \tau}^{\infty} \sum_{s=j}^{\infty} \sum_{t=s}^{\infty} \frac{t-s+1}{a_{j}}\left[p\left(t, x_{o_{1 t}}, \ldots, x_{o_{k t}}\right)-r_{t}\right] \\
& -\sum_{i=1}^{\infty} \sum_{j=m+i \tau}^{\infty} \sum_{s=j}^{\infty} \sum_{t=s}^{\infty} \frac{t-s+1}{a_{j}} \\
& \times\left[p\left(t, x_{o_{1 t}}, \ldots, x_{o_{k t}}\right)-r_{t}\right] \\
& \leq \sum_{i=1}^{\infty} \sum_{t=n+i \tau}^{\infty} \frac{W_{t}}{\left|a_{t}\right|}+\sum_{i=1}^{\infty} \sum_{t=m+i \tau}^{\infty} \frac{W_{t}}{\left|a_{t}\right|} \\
& +\sum_{i=1}^{\infty} \sum_{s=n+i \tau}^{\infty} \sum_{t=s}^{\infty} \frac{1}{\left|a_{s}\right|}\left[(t-s+1) Q_{t}+P_{t}\right] \\
& +\sum_{i=1}^{\infty} \sum_{s=m+i \tau}^{\infty} \sum_{t=s}^{\infty} \frac{1}{\left|a_{s}\right|}\left[(t-s+1) Q_{t}+P_{t}\right] \\
& +\sum_{i=1}^{\infty} \sum_{j=n+i \tau}^{\infty} \sum_{s=j}^{\infty} \sum_{t=s}^{\infty} \frac{t-s+1}{\left|a_{j}\right|}\left(R_{t}+\left|r_{t}\right|\right) \\
& +\sum_{i=1}^{\infty} \sum_{j=m+i \tau}^{\infty} \sum_{s=j}^{\infty} \sum_{t=s}^{\infty} \frac{t-s+1}{\left|a_{j}\right|}\left(R_{t}+\left|r_{t}\right|\right) \\
& \leq 2 \sum_{i=1}^{\infty} \sum_{t=T^{*}+i \tau}^{\infty} \frac{W_{t}}{\left|a_{t}\right|} \\
& +2 \sum_{i=1}^{\infty} \sum_{s=T^{*}+i \tau}^{\infty} \sum_{t=s}^{\infty} \frac{1}{\left|a_{s}\right|}\left[(t-s+1) Q_{t}+P_{t}\right] \\
& +2 \sum_{i=1}^{\infty} \sum_{j=T^{*}+i \tau}^{\infty} \sum_{s=j}^{\infty} \sum_{t=s}^{\infty} \frac{t-s+1}{\left|a_{j}\right|}\left(R_{t}+\left|r_{t}\right|\right) \\
& <\varepsilon,
\end{aligned}
$$

which yields that $S_{L}\left(\Omega_{1}(N, M)\right)$ is uniformly Cauchy.

Now we prove that $S_{L}$ is continuous in $\Omega_{1}(N, M)$. Suppose that $\left\{x^{m}\right\}_{m \in \mathbb{N}}$ is an arbitrary sequence in $\Omega_{1}(N, M)$ and $x \in \Omega_{1}(N, M)$ with $\lim _{m \rightarrow \infty} x^{m}=x$, where $x^{m}=\left\{x_{n}^{m}\right\}_{n \in \mathbb{Z}_{\beta}}$ for each $m \in \mathbb{N}$ and $x=\left\{x_{n}\right\}_{n \in \mathbb{Z}_{\beta}}$. By (12), (76), $\lim _{m \rightarrow \infty} x^{m}=$ $x$, and the continuity of $f, g, h$, and $p$, we get that for given $\varepsilon>0$, there exist $T_{1}, T_{2}, T_{3}, T_{4}$, and $T_{5} \in \mathbb{N}$ with $T_{5}>T_{4}>$ $T_{3}>T_{2}>T_{1}>T$ satisfying

$$
\begin{aligned}
& \sum_{i=T_{1}}^{\infty} \sum_{t=T+i \tau}^{\infty} \frac{W_{t}}{\left|a_{t}\right|} \\
& \quad+\sum_{i=T_{1}}^{\infty} \sum_{s=T+i \tau}^{\infty} \sum_{t=s}^{\infty} \frac{1}{\left|a_{s}\right|}\left[(t-s+1) Q_{t}+P_{t}\right]
\end{aligned}
$$




$$
\begin{aligned}
& +\sum_{i=T_{1}}^{\infty} \sum_{j=T+i \tau}^{\infty} \sum_{s=j}^{\infty} \sum_{t=s}^{\infty} \frac{t-s+1}{\left|a_{j}\right|} R_{t}<\frac{\varepsilon}{18} \\
& B=\max \left\{\frac{t-s+1}{\left|a_{s}\right|}: T \leq s \leq T+T_{2} \tau,\right. \\
& \sum_{t=T+T_{2} \tau}^{\infty} \frac{W_{t}}{\left|a_{t}\right|}+\sum_{s=T+T_{2} \tau}^{\infty} \sum_{t=s}^{\infty} \frac{1}{\left|a_{s}\right|}\left[(t-s+1) Q_{t}+P_{t}\right] \\
& \left.s \leq t \leq T+T_{3} \tau\right\}, \\
& +\sum_{j=T+T_{2} \tau}^{\infty} \sum_{\tau=j}^{\infty} \sum_{t=s}^{\infty} \frac{t-s+1}{\left|a_{j}\right|} R_{t}<\frac{\varepsilon}{18 T_{1}} \\
& \max \left\{\sum_{t=T+T_{3} \tau}^{\infty} \frac{1}{\left|a_{s}\right|}\left[(t-s+1) Q_{t}+P_{t}\right]\right. \\
& +\sum_{s=T+T_{3} \tau}^{\infty} \sum_{t=s}^{\infty} \frac{t-s+1}{\left|a_{j}\right|} R_{t} \\
& \left.: T+\tau \leq s \leq T+T_{2} \tau, T+\tau \leq j \leq T+T_{2} \tau\right\} \\
& <\frac{\varepsilon}{18 T_{1} T_{2} \tau} \\
& \max \left\{\sum_{t=T+T_{4} \tau}^{\infty} \frac{t-s+1}{\left|a_{j}\right|} R_{t}\right. \\
& \left.: T+\tau \leq j \leq T+T_{2} \tau, j \leq s \leq T+T_{3} \tau\right\} \\
& <\frac{\varepsilon}{18 T_{1} T_{2} T_{3} \tau^{2}} \\
& \sum_{i=1}^{T_{1}-1} \sum_{t=T+i \tau}^{T+T_{2} \tau-1} A\left|f\left(t, x_{b_{1 t}}^{m}, \ldots, x_{b_{k t}}^{m}\right)-f\left(t, x_{b_{1 t}}, \ldots, x_{b_{k t}}\right)\right| \\
& +\sum_{i=1}^{T_{1}-1} \sum_{s=T+i \tau}^{T+T_{2} \tau-1} \sum_{t=s}^{T+T_{3} \tau-1}\left[B \mid h\left(t, x_{d_{1 t}}^{m}, \ldots, x_{d_{k t}}^{m}\right)\right. \\
& -h\left(t, x_{d_{1 t}}, \ldots, x_{d_{k t}}\right) \\
& +A \mid g\left(t, x_{c_{1 t}}^{m}, \ldots, x_{c_{k t}}^{m}\right) \\
& \left.-g\left(t, x_{c_{1 t}}, \ldots, x_{c_{k t}}\right) \mid\right] \\
& +\sum_{i=1}^{T_{1}-1} \sum_{j=T+i \tau}^{T+T_{2} \tau-1} \sum_{s=j}^{T+T_{3} \tau-1} \sum_{t=s}^{T+T_{4} \tau-1} E \mid p\left(t, x_{o_{1 t}}^{m}, \ldots, x_{o_{k t}}^{m}\right) \\
& -p\left(t, x_{o_{1 t}}, \ldots, x_{o_{k t}}\right) \mid<\frac{\varepsilon}{18}, \\
& E=\max \left\{\frac{t-s+1}{\left|a_{j}\right|}: T \leq j \leq T+T_{2} \tau,\right. \\
& \left.j \leq s \leq T+T_{3} \tau, s \leq t \leq T+T_{4} \tau\right\} . \\
& \left\|S_{L} x^{m}-S_{L} x\right\| \\
& =\sup _{n \in \mathbb{Z}_{\beta}}\left|\left(S_{L} x^{m}\right)_{n}-\left(S_{L} x\right)_{n}\right| \\
& =\max \left\{\sup _{\beta \geq n>T}\left|\left(S_{L} x^{m}\right)_{n}-\left(S_{L} x\right)_{n}\right|,\right. \\
& \left.\sup _{n \geq T}\left|\left(S_{L} x^{m}\right)_{n}-\left(S_{L} x\right)_{n}\right|\right\} \\
& =\sup _{n \geq T} \mid L-\sum_{i=1}^{\infty} \sum_{t=n+i \tau}^{\infty} \frac{1}{a_{t}} f\left(t, x_{b_{1 t}}^{m}, \ldots, x_{b_{k t}}^{m}\right) \\
& -\sum_{i=1}^{\infty} \sum_{s=n+i \tau}^{\infty} \sum_{t=s}^{\infty} \frac{1}{a_{s}}\left[(t-s+1) h\left(t, x_{d_{1 t}}^{m}, \ldots, x_{d_{k t}}^{m}\right)\right. \\
& \left.-g\left(t, x_{c_{1 t}}^{m}, \ldots, x_{c_{k t}}^{m}\right)\right] \\
& -\sum_{i=1}^{\infty} \sum_{j=n+i \tau}^{\infty} \sum_{s=j}^{\infty} \sum_{t=s}^{\infty} \frac{t-s+1}{a_{j}}\left[p\left(t, x_{o_{1 t}}^{m}, \ldots, x_{o_{k t}}^{m}\right)-r_{t}\right] \\
& -\left(L-\sum_{i=1}^{\infty} \sum_{t=n+i \tau}^{\infty} \frac{1}{a_{t}} f\left(t, x_{b_{1 t}}, \ldots, x_{b_{k t}}\right)\right. \\
& -\sum_{i=1}^{\infty} \sum_{s=n+i \tau}^{\infty} \sum_{t=s}^{\infty} \frac{1}{a_{s}}\left[(t-s+1) h\left(t, x_{d_{1 t}}, \ldots, x_{d_{k t}}\right)\right. \\
& \left.-g\left(t, x_{c_{1 t}}, \ldots, x_{c_{k t}}\right)\right] \\
& -\sum_{i=1}^{\infty} \sum_{j=n+i \tau}^{\infty} \sum_{s=j}^{\infty} \sum_{t=s}^{\infty} \frac{t-s+1}{a_{j}} \\
& \left.\times\left[p\left(t, x_{o_{1 t}}, \ldots, x_{o_{k t}}\right)-r_{t}\right]\right) \\
& \leq \sum_{i=1}^{\infty} \sum_{t=T+i \tau}^{\infty} \frac{1}{\left|a_{t}\right|}\left|f\left(t, x_{b_{1 t}}^{m}, \ldots, x_{b_{k t}}^{m}\right)-f\left(t, x_{b_{1 t}}, \ldots, x_{b_{k t}}\right)\right| \\
& A=\max \left\{\frac{1}{\left|a_{s}\right|}: T \leq s \leq T+T_{2} \tau\right\}, \\
& +\sum_{i=1}^{\infty} \sum_{s=T+i \tau}^{\infty} \sum_{t=s}^{\infty} \frac{1}{\left|a_{s}\right|}\left[(t-s+1) \mid h\left(t, x_{d_{1 t}}^{m}, \ldots, x_{d_{k t}}^{m}\right)\right.
\end{aligned}
$$


24

Abstract and Applied Analysis

$$
\begin{aligned}
& -h\left(t, x_{d_{1 t}}, \ldots, x_{d_{k t}}\right) \\
& +\mid g\left(t, x_{c_{1 t}}^{m}, \cdots, x_{c_{k t}}^{m}\right) \\
& \left.-g\left(t, x_{c_{1 t}}, \ldots, x_{c_{k t}}\right) \mid\right] \\
& \left.-g\left(t, x_{c_{1 t}}, \ldots, x_{c_{k t}}\right) \mid\right] \\
& +\sum_{i=T_{1} s=T+i i t}^{\infty} \sum^{\infty} \sum_{t=s}^{\infty} \frac{1}{\left|a_{s}\right|}\left[(t-s+1) \mid h\left(t, x_{d_{1 t}}^{m}, \ldots, x_{d_{k t}}^{m}\right)\right. \\
& +\sum_{i=T_{1}}^{\infty} \sum_{j=T+i \tau}^{\infty} \sum^{\infty} \sum_{s=j t=s}^{\infty} \frac{t-s+1}{\left|a_{j}\right|} \mid p\left(t, x_{o_{1 t}}^{m}, \ldots, x_{o_{k t}}^{m}\right) \\
& -h\left(t, x_{d_{1 t}}, \ldots, x_{d_{k t}}\right) \mid \\
& -p\left(t, x_{o_{1 t}}, \ldots, x_{o_{k t}}\right) \mid \\
& +\mid g\left(t, x_{c_{1 t}}^{m}, \ldots, x_{c_{k t}}^{m}\right) \\
& \left.-g\left(t, x_{c_{1 t}}, \ldots, x_{c_{k t}}\right) \mid\right] \\
& \leq \sum_{i=1}^{T_{1}-1} \sum_{t=T+i \tau}^{T+T_{2} \tau-1} \frac{1}{\left|a_{t}\right|} \mid f\left(t, x_{b_{1 t}}^{m}, \ldots, x_{b_{k t}}^{m}\right) \\
& -f\left(t, x_{b_{1 t}}, \ldots, x_{b_{k t}}\right) \mid \\
& +\sum_{i=1}^{T_{1}-1} \sum_{t=T+T_{2} \tau}^{\infty} \frac{1}{\left|a_{t}\right|} \mid f\left(t, x_{b_{1 t}}^{m}, \ldots, x_{b_{k t}}^{m}\right) \\
& -f\left(t, x_{b_{1 t}}, \ldots, x_{b_{k t}}\right) \\
& +\sum_{i=T_{1}}^{\infty} \sum_{t=T+i \tau}^{\infty} \frac{1}{\left|a_{t}\right|} \mid f\left(t, x_{b_{1 t}}^{m}, \ldots, x_{b_{k t}}^{m}\right) \\
& -f\left(t, x_{b_{1 t}}, \ldots, x_{b_{k t}}\right) \\
& +\sum_{i=1}^{T_{1}-1 T+\sum_{s=T+i \tau}} \sum_{t=s}^{T+T_{2} \tau-1 T+T_{3} \tau-1} \frac{1}{\left|a_{s}\right|} \\
& \times\left[(t-s+1) \mid h\left(t, x_{d_{1 t}}^{m}, \ldots, x_{d_{k t}}^{m}\right)\right. \\
& -h\left(t, x_{d_{1 t}}, \ldots, x_{d_{k t}}\right) \\
& +\mid g\left(t, x_{c_{1 t}}^{m}, \ldots, x_{c_{k t}}^{m}\right) \\
& \left.\left.-g\left(t, x_{c_{1 t}}, \ldots, x_{c_{k t}}\right)\right]\right] \\
& +\sum_{i=1}^{T_{1}-1 T \sum_{s=T+i \tau}} \sum_{t=T+T_{3} \tau}^{\infty} \frac{1}{\left|a_{s}\right|} \\
& \times\left[(t-s+1) \mid h\left(t, x_{d_{1 t}}^{m}, \ldots, x_{d_{k t}}^{m}\right)\right. \\
& -h\left(t, x_{d_{1 t}}, \ldots, x_{d_{k t}}\right) \mid \\
& +\mid g\left(t, x_{c_{1 t}}^{m}, \ldots, x_{c_{k t}}^{m}\right) \\
& \left.-g\left(t, x_{c_{1 t}}, \ldots, x_{c_{k t}}\right)\right] \\
& +\sum_{i=1}^{T_{1}-1} \sum_{s=T+T_{2} \tau}^{\infty} \sum_{t=s}^{\infty} \frac{1}{\left|a_{s}\right|}\left[(t-s+1) \mid h\left(t, x_{d_{1 t}}^{m}, \ldots, x_{d_{k t}}^{m}\right)\right. \\
& -h\left(t, x_{d_{1 t}}, \ldots, x_{d_{k t}}\right) \mid \\
& +\mid g\left(t, x_{c_{1 t}}^{m}, \ldots, x_{c_{k t}}^{m}\right) \\
& +\sum_{i=1}^{T_{1}-1} \sum_{j=T+i \tau}^{T+T_{2} \tau-1} \sum_{s=j}^{T+T_{3} \tau-1} \sum_{t=s}^{T+T_{4} \tau-1} \frac{t-s+1}{\left|a_{j}\right|} \\
& \times \mid p\left(t, x_{o_{1 t}}^{m}, \ldots, x_{o_{k t}}^{m}\right) \\
& -p\left(t, x_{o_{1 t}}, \ldots, x_{o_{k t}}\right) \mid \\
& +\sum_{i=1}^{T_{1}-1} \sum_{j=T+i \tau}^{T+T_{2} \tau-1} \sum_{s=j}^{T+T_{3} \tau-1} \sum_{t=T+T_{4} \tau}^{\infty} \frac{t-s+1}{\left|a_{j}\right|} \\
& \times \mid p\left(t, x_{o_{1 t}}^{m}, \ldots, x_{o_{k t}}^{m}\right) \\
& -p\left(t, x_{o_{1 t}}, \ldots, x_{o_{k t}}\right) \\
& +\sum_{i=1}^{T_{1}-1} \sum_{j=T+i \tau}^{T+T_{2} \tau-1} \sum_{s=T+T_{3} \tau}^{\infty} \sum_{t=s}^{\infty} \frac{t-s+1}{\left|a_{j}\right|} \\
& \times \mid p\left(t, x_{o_{1 t}}^{m}, \ldots, x_{o_{k t}}^{m}\right) \\
& -p\left(t, x_{o_{1 t}}, \ldots, x_{o_{k t}}\right) \mid \\
& +\sum_{i=1}^{T_{1}-1} \sum_{j=T+T_{2} \tau}^{\infty} \sum_{s=j}^{\infty} \sum_{t=s}^{\infty} \frac{t-s+1}{\left|a_{j}\right|} \\
& \times \mid p\left(t, x_{o_{1 t}}^{m}, \ldots, x_{o_{k t}}^{m}\right) \\
& -p\left(t, x_{o_{1 t}}, \ldots, x_{o_{k t}}\right) \mid \\
& +\sum_{i=T_{1}}^{\infty} \sum_{j=T+i \tau}^{\infty} \sum_{s=j}^{\infty} \sum_{t=s}^{\infty} \frac{t-s+1}{\left|a_{j}\right|} \\
& \times \mid p\left(t, x_{o_{1 t}}^{m}, \ldots, x_{o_{k t}}^{m}\right) \\
& -p\left(t, x_{o_{1 t}}, \ldots, x_{o_{k t}}\right) \mid \\
& \leq \sum_{i=1}^{T_{1}-1 T+\sum_{t=T+i \tau} \tau+T_{2}-1} A\left|f\left(t, x_{b_{1 t}}^{m}, \ldots, x_{b_{k t}}^{m}\right)-f\left(t, x_{b_{1 t}}, \ldots, x_{b_{k t}}\right)\right| \\
& +\sum_{i=1}^{T_{1}-1 T} \sum_{s=T+i \tau}^{T+T_{2} \tau-1} \sum_{t=s}^{T+T_{3} \tau-1}\left[B \mid h\left(t, x_{d_{1 t}}^{m}, \ldots, x_{d_{k t}}^{m}\right)\right. \\
& -h\left(t, x_{d_{1 t}}, \ldots, x_{d_{k t}}\right)
\end{aligned}
$$




$$
\begin{aligned}
& +A \mid g\left(t, x_{c_{1 t}}^{m}, \ldots, x_{c_{k t}}^{m}\right) \\
& \left.-g\left(t, x_{c_{1 t}}, \ldots, x_{c_{k t}}\right) \mid\right] \\
& +\sum_{i=1}^{T_{1}-1 T+\sum_{j=T+i \tau}} \sum_{s=j} \sum_{t=s}^{\tau-1 T+T_{3} \tau-1 T+T_{4} \tau-1} E \mid p\left(t, x_{o_{1 t}}^{m}, \cdots, x_{o_{k t}}^{m}\right) \\
& -p\left(t, x_{o_{1 t}}, \cdots, x_{o_{k t}}\right) \\
& +2 \sum_{i=1}^{T_{1}-1} \sum_{t=T+T_{2} \tau}^{\infty} \frac{W_{t}}{\left|a_{t}\right|}+2 \sum_{i=T_{1}}^{\infty} \sum_{t=T+i \tau}^{\infty} \frac{W_{t}}{\left|a_{t}\right|} \\
& +2 \sum_{i=1}^{T_{1}-1} \sum_{s=T+i \tau} \sum_{t=T+T_{3} \tau}^{\infty} \frac{1}{\left|a_{s}\right|}\left[(t-s+1) Q_{t}+P_{t}\right] \\
& +2 \sum_{i=1}^{T_{1}-1} \sum_{s=T+T_{2} \tau}^{\infty} \sum_{t=s}^{\infty} \frac{1}{\left|a_{s}\right|}\left[(t-s+1) Q_{t}+P_{t}\right] \\
& +2 \sum_{i=T_{1}}^{\infty} \sum_{s=T+i \tau}^{\infty} \sum_{t=s}^{\infty} \frac{1}{\left|a_{s}\right|}\left[(t-s+1) Q_{t}+P_{t}\right] \\
& +2 \sum_{i=1}^{T_{1}-1} \sum_{j=T+i \tau}^{T+T_{2} \tau-1} \sum_{s=j}^{T+T_{3} \tau-1} \sum_{t=T+T_{4} \tau}^{\infty} \frac{t-s+1}{\left|a_{j}\right|} R_{t} \\
& +2 \sum_{i=1}^{T_{1}-1} \sum_{j=T+i \tau}^{T+T_{2} \tau-1} \sum_{s=T+T_{3} \tau}^{\infty} \sum_{t=s}^{\infty} \frac{t-s+1}{\left|a_{j}\right|} R_{t} \\
& +2 \sum_{i=1}^{T_{1}-1} \sum_{j=T+T_{2} \tau}^{\infty} \sum_{s=j}^{\infty} \sum_{t=s}^{\infty} \frac{t-s+1}{\left|a_{j}\right|} R_{t} \\
& +2 \sum_{i=T_{1}}^{\infty} \sum_{j=T+i \tau}^{\infty} \sum_{s=j}^{\infty} \sum_{t=s}^{\infty} \frac{t-s+1}{\left|a_{j}\right|} R_{t} \\
& <\frac{\varepsilon}{18}+\frac{2\left(T_{1}-1\right)\left(T_{2}-1\right) \tau \varepsilon}{18 T_{1} T_{2} \tau} \\
& +\frac{2\left(T_{1}-1\right) \varepsilon}{18 T_{1}} \\
& +\frac{2\left(T_{1}-1\right)\left(T_{2}-1\right)\left(T_{3}-1\right) \tau^{2} \varepsilon}{18 T_{1} T_{2} T_{3} \tau^{2}} \\
& +\frac{2 \varepsilon}{18}<\varepsilon, \quad \forall m \geq T_{5} \text {, }
\end{aligned}
$$

which yields that $S_{L}$ is continuous in $\Omega_{1}(N, M)$. It follows from Lemmas 2 and 4 that $S_{L}$ has a fixed point $x=\left\{x_{n}\right\}_{n \in \mathbb{Z}_{\beta}} \in$ $\Omega_{1}(N, M)$; that is,

$$
\begin{aligned}
x_{n}= & L-\sum_{i=1}^{\infty} \sum_{t=n+i \tau}^{\infty} \frac{1}{a_{t}} f\left(t, x_{b_{1 t}}, \ldots, x_{b_{k t}}\right) \\
& -\sum_{i=1}^{\infty} \sum_{s=n+i \tau}^{\infty} \sum_{t=s}^{\infty} \frac{1}{a_{s}}[(t-s+1)
\end{aligned}
$$

$$
\begin{gathered}
\times h\left(t, x_{d_{1 t}}, \ldots, x_{d_{k t}}\right) \\
\left.-g\left(t, x_{c_{1 t}}, \ldots, x_{c_{k t}}\right)\right] \\
+\sum_{i=1}^{\infty} \sum_{j=n+i \tau}^{\infty} \sum_{s=j}^{\infty} \frac{t-s+1}{a_{j}} \\
\times\left[p\left(t, x_{o_{1 t}}, \ldots, x_{o_{k t}}\right)-r_{t}\right], \\
x_{n-\tau}=L n \geq T+\tau, \\
-\sum_{i=1}^{\infty} \sum_{t=n+(i-1) \tau}^{\infty} \frac{1}{a_{t}} f\left(t, x_{b_{1 t}}, \ldots, x_{b_{k t}}\right) \\
\sum_{i=1}^{\infty} \sum_{s=n+(i-1) \tau}^{\infty} \frac{1}{a_{s}} \quad \times\left[(t-s+1) h\left(t, x_{d_{1 t}}, \ldots, x_{d_{k t}}\right)\right. \\
+\sum_{i=1}^{\infty} \sum_{j=n+(i-1) \tau}^{\infty} \sum_{s=j}^{\infty} \sum_{t=s}^{\infty} \frac{t-s+1}{a_{j}} \quad \times\left[p\left(t, x_{o_{1 t}}, \ldots, x_{o_{k t}}\right)-r_{t}\right], \\
\quad \forall n \geq T+\tau,
\end{gathered}
$$

which imply that

$$
\begin{aligned}
& \Delta\left(x_{n}-x_{n-\tau}\right) \\
& =-\frac{1}{a_{n}} f\left(n, x_{b_{1 n}}, \ldots, x_{b_{k n}}\right) \\
& \quad-\sum_{t=n}^{\infty} \frac{1}{a_{n}}\left[(t-n+1) h\left(t, x_{d_{1 t}}, \ldots, x_{d_{k t}}\right)\right. \\
& \left.\quad-g\left(t, x_{c_{1 t}}, \ldots, x_{c_{k t}}\right)\right] \\
& +\sum_{s=n}^{\infty} \sum_{t=s}^{\infty} \frac{t-s+1}{a_{n}}\left[p\left(t, x_{o_{1 t}}, \ldots, x_{o_{k t}}\right)-r_{t}\right], \\
& \forall n \geq T+\tau,
\end{aligned}
$$

which yields that

$$
\begin{aligned}
& \Delta^{3}\left(a_{n} \Delta\left(x_{n}-x_{n-\tau}\right)\right)+\Delta^{3} f\left(n, x_{b_{1 n}}, \ldots, x_{b_{k n}}\right) \\
& +\Delta^{2} g\left(n, x_{c_{1 n}}, \ldots, x_{c_{k n}}\right)+\Delta h\left(n, x_{d_{1 n}}, \ldots, x_{d_{k n}}\right) \\
& \quad+p\left(n, x_{o_{1 n}}, \ldots, x_{o_{k n}}\right)=r_{n}, \quad \forall n \geq T+\tau ;
\end{aligned}
$$

that is, $x=\left\{x_{n}\right\}_{n \in \mathbb{Z}_{\beta}} \in \Omega_{1}(N, M)$ is a bounded positive solution of (2). The rest of the proof is similar to that of Theorem 6 and is omitted. This is completes proof. 


\section{Applications}

Now we display nine examples as applications of the results presented in Section 3.

Example 1. Consider the fourth order nonlinear neutral delay difference equation:

$$
\begin{aligned}
& \Delta^{3}\left(\left(n^{5}+2 n\right) \Delta\left(x_{n}+\frac{(-1)^{n} n^{2}}{4 n^{2}+n+3} x_{n-\tau}\right)\right) \\
& +\Delta^{3}\left(n x_{n^{2}-n}^{17}+\sqrt{n} x_{n^{2}-n}^{5}-\sqrt{n-3}\right) \\
& +\Delta^{2}\left(\frac{(-1)^{n} x_{n-3}^{21}+5 n^{2}+1}{\sqrt{n^{7}-17}\left(x_{n-3}^{4}+3 n^{2}+2\right)}\right) \\
& +\Delta\left(\frac{12 \sqrt{n}-x_{2 n-1}^{15} \cos ^{2}\left(n^{3} x_{2 n-1}^{5}\right)}{2+n^{5}\left|x_{2 n-1}\right|}\right) \\
& +\frac{(-1)^{n-1} n \sin ^{11}\left(n x_{n^{3}-3 n}^{9}\right)}{\sqrt{n^{2}+2 n+5}\left(1+x_{n^{3}-3 n}^{22}\right)+n^{5}} \\
& =\frac{4 n^{3}+2 n-1}{(n+1)^{12} \sqrt{3 n^{2}+7}},
\end{aligned}
$$

$\forall n \geq 7$.

It follows from Theorem 6 that (88) possesses uncountably many bounded positive solutions in $\Omega_{1}(N, M)$.

Example 2. Consider the fourth order nonlinear neutral delay difference equation:

$$
\begin{aligned}
& \Delta^{3}\left((-1)^{n+1} n^{5 / 2} \Delta\left(x_{n}+\frac{6 n-3}{10 n+2} x_{n-\tau}\right)\right) \\
& +\Delta^{3}\left(\frac{x_{n^{3}-5}^{5}+2 n^{3}}{x_{n^{3}-5}^{8}+(n+3)^{4}}\right) \\
& +\Delta^{2}\left(\frac{(-1)^{n-1} n^{2}}{x_{2 n-1}^{14}+n^{5}+\left|\cos \left(x_{2 n-1}^{2} n^{3}\right)\right|}\right) \\
& +\Delta\left(\frac{x_{n-1}^{4} \sin \sqrt{n+x_{n-1}^{2}}-n^{4}}{n^{7}+x_{n-1}^{6} \cos ^{4} \sqrt{\left|x_{n-1}^{3}\right|+n}}\right) \\
& +\frac{x_{5 n-9}^{8} n^{6}}{n^{9}+\left|n x_{5 n-9} \sin ^{3}\left(n^{2}+1\right)\right|} \\
& =\frac{\cos \left(n^{3}-2 n+3\right)}{n^{4}+\sqrt{3 n-1}}
\end{aligned}
$$

$\forall n \geq 1$.

It follows from Theorem 7 that (89) possesses uncountably many bounded positive solutions in $\Omega_{1}(N, M)$.
Example 3. Consider the fourth order nonlinear neutral delay difference equation:

$$
\begin{aligned}
\Delta^{3}( & \left(n^{7}-n^{3}+2\right) \\
& \left.\times \Delta\left(x_{n}-\frac{4 n^{2} \cos ^{4}\left(3 n^{2}+2\right)}{5 n^{2}+2 n} x_{n-\tau}\right)\right) \\
+ & \Delta^{3}\left(\sqrt{n-9} x_{n^{3}-5}^{3} x_{n+11}^{5}\right) \\
+ & \Delta^{2}\left(\frac{\sqrt{n+3} x_{n^{3}-4}^{21}-\sqrt{2 n+5} x_{n^{2}-1}^{12}}{n^{5}+3 n x_{n^{3}-4}^{2} x_{n^{2}-1}^{2}}\right) \\
+ & \Delta\left(\frac{\left(x_{n-7}+x_{n^{4}-1}+n\right)^{4}}{\left|\left(n+x_{n-7}\right)^{5}\left(n+x_{n^{4}-1}\right)^{3}\right|+1}\right) \\
+ & \frac{n^{2} x_{n^{2}-3}^{3} x_{n-4}^{5}+\ln n}{n^{2}+x_{n-4}^{4}+\left(n+x_{n^{2}-3}\right)^{6}} \\
= & \frac{(-1)^{n+1}\left(4 n^{5}-3\right)}{n^{9}+4 n^{2}+5},
\end{aligned}
$$

$\forall n \geq 9$.

Theorem 8 implies that (90) possesses uncountably many bounded positive solutions in $\Omega_{1}(N, M)$.

Example 4. Consider the fourth order nonlinear neutral delay difference equation:

$$
\begin{aligned}
& \Delta^{3}\left(n^{3} \Delta\left(x_{n}+\sqrt{\ln \left(3 n^{3}-134 n\right)} x_{n-\tau}\right)\right) \\
& +\Delta^{3}\left(\frac{2 n+x_{2 n-3}^{3} \cos \sqrt{n}}{\left(n+x_{2 n-3}\right)^{4}(n+1)^{5}+72}\right) \\
& +\Delta^{2}\left(\frac{n x_{n^{5}}^{7} \sin ^{3}\left(n^{2} x_{n^{5}}^{3}\right)}{(\sqrt{n+2}+3)^{6}}\right) \\
& +\Delta\left(\frac{x_{n+6}^{2}-(-1)^{n+1} n}{\sqrt{n^{2}+1}+\left(x_{n+6}+\sqrt{n}\right)^{10}}\right) \\
& +\frac{n^{2}+x_{2 n-18}^{27}}{n^{13}+x_{2 n-18}^{2}\left|\cos ^{3}\left(n x_{2 n-18}^{4}\right)\right|} \\
& =\frac{n^{7}-n^{11}-2}{n^{15}+36},
\end{aligned}
$$

$\forall n \geq 15$.

Theorem 9 yields that (91) has uncountably many bounded positive solutions in $l_{\beta}^{\infty}$. 
Example 5. Consider the fourth order nonlinear neutral delay difference equation:

$$
\begin{aligned}
& \Delta^{3}\left((-1)^{n} n \ln ^{2} n \Delta\left(x_{n}+\left(1+\frac{1}{n}\right)^{n} x_{n-\tau}\right)\right) \\
& +\Delta^{3}\left(\frac{\sqrt{n} x_{n-4}^{3}-x_{n-4}^{2}+1}{n^{5}+\ln \left(1+x_{n-4}^{2}\right)}\right) \\
& +\Delta^{2}\left(\frac{n^{2} x_{n-9}^{3}-n x_{n-9}-\sqrt{n}}{n^{8}+\left(x_{n-9}^{4}-n\right)^{4}}\right) \\
& +\Delta\left(\frac{x_{n-6}^{5}-(-1)^{n} n}{n^{6}+\ln 3\left(n+x_{n-6}^{8}\right)}\right) \\
& +\frac{\cos \left(n-\ln \left(1+x_{n-7}^{2}\right)\right)}{n^{5}+\cos ^{3}\left(n x_{n-7}^{7}\right)+1} \\
& =\frac{n^{2}-n+(-1)^{n}}{n^{9}+3 n+1},
\end{aligned}
$$

$\forall n \geq 10$.

Theorem 10 guarantees that (92) possesses uncountably many bounded positive solutions in $l_{\beta}^{\infty}$.

Example 6. Consider the fourth order nonlinear neutral delay difference equation:

$$
\begin{aligned}
& \Delta^{3}\left(\frac{n^{4}+2 n}{\ln (n+5)} \Delta\left(x_{n}-(n-16)^{3} x_{n-\tau}\right)\right) \\
& +\Delta^{3}\left(\sqrt{n^{2}-2 n} \cos ^{2}\left(n x_{n-5}^{2}\right)\right) \\
& +\Delta^{2}\left(\frac{x_{n^{2}-2 n+3}^{3} \ln ^{2}(n-13)}{n^{11}+\left|x_{n^{2}-2 n+3}+1\right|}\right) \\
& +\Delta\left(\frac{n x_{n-19}^{4} \sin \left(x_{n-19}^{2}+n\right)}{n^{9}+2 n+x_{n-19}^{6}}\right) \\
& +\frac{n^{2} \sin ^{16}\left(n+x_{n-13}^{4}\right)}{\left(n+x_{n-13}^{2}\right)^{8}} \\
& =\frac{(-1)^{n+1}\left(n^{3}+11\right)}{(n+2)^{5}(n+5)^{4}}, \quad \forall n \geq 18 .
\end{aligned}
$$

Theorem 11 ensures that (93) possesses uncountably many bounded positive solutions in $l_{\beta}^{\infty}$.
Example 7. Consider the fourth order nonlinear neutral delay difference equation:

$$
\begin{aligned}
& \Delta^{3}\left(-n^{8} \Delta\left(x_{n}-\left(3-\sin ^{9} n\right) x_{n-\tau}\right)\right) \\
& +\Delta^{3}\left(\frac{n^{5} \ln \left(n+x_{n-30}^{2}\right)-x_{n-30}^{3}}{n^{25}+x_{n-30}^{8}}\right) \\
& +\Delta^{2}\left(\frac{n^{3} x_{n^{2}-1}^{3}-n x_{n^{2}-1}^{2}-\cos \left(n^{5}\right)}{n^{81}+\left(n x_{n^{2}-1}^{3}-1\right)^{2}}\right) \\
& +\Delta\left(\frac{n^{6} x_{n-2}^{3}-n+3}{n^{16}+\left(n^{3}-x_{n-2}^{7}\right)^{2}}\right) \\
& +\frac{n^{8}+3 n^{5} x_{n-4}^{2}-x_{n-4}^{3}}{n^{58}+n^{22}-\sin \left(n^{5} x_{n-4}^{3}\right)} \\
& =\frac{n^{25}+(-1)^{n} n^{11}-1}{n^{91}+33 n+5}, \quad \forall n \geq 1 .
\end{aligned}
$$

Theorem 12 guarantees that (94) possesses uncountably many bounded positive solutions in $l_{\beta}^{\infty}$.

Example 8. Consider the fourth order nonlinear neutral delay difference equation:

$$
\begin{aligned}
\Delta^{3} & \left((-1)^{n-1}(n+2)^{3} \Delta\left(x_{n}+x_{n-\tau}\right)\right) \\
+ & \Delta^{3}\left(\frac{n^{4} x_{n^{2}-9}^{5} x_{n-4}^{2}}{n^{3}+\left|x_{n^{2}-9}^{25}-n x_{n-4}^{37}\right|}\right) \\
+ & \Delta^{2}\left(\frac{n^{4}+x_{n-1}^{2} x_{n^{3}-3}^{3}}{n^{9}+\ln ^{3}\left(n^{2}+x_{n^{3}-3}^{6}\right)}\right) \\
& +\Delta\left(\frac{\sin \left(n^{9} x_{n-2}^{8} x_{5 n-6}^{9}\right)}{n^{5}+\left(n+2 x_{n-2}^{82} x_{5 n-6}^{19}\right)^{2}}\right) \\
& +\frac{\ln \left(n^{2}+x_{n^{2}-17^{6}}^{6} x_{n-6}^{14}\right)}{n^{16}+\left(2 x_{n^{2}-17}+x_{n-6}\right)^{2}} \\
= & \frac{(-1)^{n(n+1) / 2} \cos ^{7}\left(3 n^{5}+1\right)}{n^{5}+n^{3}+3},
\end{aligned}
$$

$\forall n \geq 8$

It follows from Theorem 13 that (95) possesses uncountably many bounded positive solutions in $\Omega(N, M)$. 
Example 9. Consider the fourth order nonlinear neutral delay difference equation:

$$
\begin{aligned}
\Delta^{3} & \left(n^{17} \Delta\left(x_{n}-x_{n-\tau}\right)\right) \\
+ & \Delta^{3}\left(\sqrt{n} x_{9 n-5}^{3} x_{2 n-1}^{2}-\sin ^{3}\left(\arctan \left(n^{2}+1\right)\right)\right) \\
+ & \Delta^{2}\left(\frac{n^{3}+x_{n-7}^{4} x_{n^{2}-2}^{5}}{\left(n+x_{n-7}^{6}\right)^{13}+\left|x_{n-7}-n x_{n^{2}-2}^{2}\right|}\right) \\
+ & \Delta\left(\frac{x_{n-10}^{3} \ln ^{4}\left(n^{2}+x_{n^{3}-3}^{2}\right)}{\left(n+x_{n-10}^{2} x_{n^{3}-3}^{2}\right)^{9}(n+2)^{31}}\right) \\
+ & \frac{\left(3-n^{2}\right) x_{3 n-2}^{2}-n x_{n-9}^{9}}{n^{60}+\left|x_{3 n-2} x_{n-9}\right|} \\
& =\frac{(-1)^{n}\left(n^{2}-1\right) \cos ^{5}(n-\sqrt{n})}{n^{19}+15 n^{8}+1}, \quad \forall n \geq 11 .
\end{aligned}
$$

Theorem 14 means that (96) possesses uncountably many bounded positive solutions in $\Omega(N, M)$.

\section{Conflict of Interests}

The authors declare that there is no conflict of interests regarding the publication of this paper.

\section{Acknowledgments}

The authors would like to express their thanks to the anonymous referee for her/his valuable suggestions and comments. This research was supported by the Science Research Foundation of Educational Department of Liaoning Province (L2012380).

\section{References}

[1] M. H. Abu-Risha, "Oscillation of second-order linear difference equations," Applied Mathematics Letters, vol. 13, no. 1, pp. 129$135,2000$.

[2] R. P. Agarwal, Difference Equations and Inequalities, Marcel Dekker, New York, NY, USA, 2nd edition, 2000.

[3] R. P. Agarwal, S. R. Grace, and D. O’Regan, "Nonoscillatory solutions for discrete equations," Computers \& Mathematics with Applications, vol. 45, no. 6-9, pp. 1297-1302, 2003.

[4] R. P. Agarwal, M. M. S. Manuel, and E. Thandapani, "Oscillatory and nonoscillatory behavior of second-order neutral delay difference equations," Applied Mathematics Letters, vol. 10, no. 2, pp. 103-109, 1997.

[5] S. S. Cheng and W. T. Patula, "An existence theorem for a nonlinear difference equation," Nonlinear Analysis: Theory, Methods \& Applications, vol. 20, no. 3, pp. 193-203, 1993.

[6] S. S. Cheng, H. J. Li, and W. T. Patula, "Bounded and zero convergent solutions of second-order difference equations," Journal of Mathematical Analysis and Applications, vol. 141, no. 2, pp. 463-483, 1989.
[7] W. T. Li and S. S. Cheng, "Oscillation criteria for a nonlinear difference equation," Computers \& Mathematics with Applications, vol. 36, no. 8, pp. 87-94, 1998.

[8] W.-T. Li and S.-S. Cheng, "Remarks on two recent oscillation theorems for second-order linear difference equations," Applied Mathematics Letters, vol. 16, no. 2, pp. 161-163, 2003.

[9] W.-T. Li, X.-L. Fan, and C.-k. Zhong, "On unbounded positive solutions of second-order difference equations with a singular nonlinear term," Journal of Mathematical Analysis and Applications, vol. 246, no. 1, pp. 80-88, 2000.

[10] W.-T. Li and S. H. Saker, "Oscillation of second-order sublinear neutral delay difference equations," Applied Mathematics and Computation, vol. 146, no. 2-3, pp. 543-551, 2003.

[11] H.-J. Li and C.-C. Yeh, "Oscillation criteria for second-order neutral delay difference equations," Computers \& Mathematics with Applications, vol. 36, no. 10-12, pp. 123-132, 1998.

[12] X. Li and D. Zhu, "New results for the asymptotic behavior of a nonlinear second-order difference equation," Applied Mathematics Letters, vol. 16, no. 5, pp. 627-633, 2003.

[13] H. Liang and P. Weng, "Existence and multiple solutions for a second-order difference boundary value problem via critical point theory," Journal of Mathematical Analysis and Applications, vol. 326, no. 1, pp. 511-520, 2007.

[14] Z. Liu, S. M. Kang, and J. S. Ume, "Existence of uncountably many bounded nonoscillatory solutions and their iterative approximations for second order nonlinear neutral delay difference equations," Applied Mathematics and Computation, vol. 213 , no. 2, pp. 554-576, 2009

[15] Z. Liu, L. Wang, G. I. Kim, and S. M. Kang, "Existence of uncountably many bounded positive solutions for a third order nonlinear neutral delay difference equation," Computers \& Mathematics with Applications, vol. 60, no. 8, pp. 2399-2416, 2010.

[16] Z. Liu, Y. Xu, and S. M. Kang, "Global solvability for a second order nonlinear neutral delay difference equation," Computers \& Mathematics with Applications, vol. 57, no. 4, pp. 587-595, 2009.

[17] J. W. Luo and D. D. Bainov, "Oscillatory and asymptotic behavior of second-order neutral difference equations with maxima," Journal of Computational and Applied Mathematics, vol. 131, no. 1-2, pp. 333-341, 2001.

[18] M. Ma, H. Tang, and W. Luo, "Periodic solutions for nonlinear second-order difference equations," Applied Mathematics and Computation, vol. 184, no. 2, pp. 685-694, 2007.

[19] Q. Meng and J. Yan, "Bounded oscillation for second-order nonlinear delay differential equation in a critical state," Journal of Computational and Applied Mathematics, vol. 197, no. 1, pp. 204-211, 2006.

[20] M. Migda and J. Migda, "Asymptotic properties of solutions of second-order neutral difference equations," Nonlinear Analysis: Theory, Methods and Applications, vol. 63, no. 5-7, pp. 789-799, 2005.

[21] S. H. Saker, "Oscillation of third-order difference equations," Portugaliae Mathematica, vol. 61, no. 3, pp. 249-257, 2004.

[22] S. H. Saker, "New oscillation criteria for second-order nonlinear neutral delay difference equations," Applied Mathematics and Computation, vol. 142, no. 1, pp. 99-111, 2003.

[23] M.-C. Tan and E.-H. Yang, "Oscillation and nonoscillation theorems for second order nonlinear difference equations," Journal of Mathematical Analysis and Applications, vol. 276, no. 1, pp. 239-247, 2002. 
[24] X. H. Tang, "Bounded oscillation of second-order delay difference equations of unstable type," Computers \& Mathematics with Applications, vol. 44, no. 8-9, pp. 1147-1156, 2002.

[25] E. Thandapani, M. M. S. Manuel, J. R. Graef, and P. W. Spikes, "Monotone properties of certain classes of solutions of secondorder difference equations," Computers \& Mathematics with Applications, vol. 36, pp. 291-297, 1998.

[26] Y. Tian and W. Ge, "Multiple positive solutions of boundary value problems for second-order discrete equations on the halfline," Journal of Difference Equations and Applications, vol. 12, no. 2, pp. 191-208, 2006.

[27] J. Yan and B. Liu, "Asymptotic behavior of a nonlinear delay difference equation," Applied Mathematics Letters, vol. 8, no. 6, pp. 1-5, 1995.

[28] B. G. Zhang, "Oscillation and asymptotic behavior of second order difference equations," Journal of Mathematical Analysis and Applications, vol. 173, no. 1, pp. 58-68, 1993.

[29] Z. Zhang, J. Chen, and C. Zhang, "Oscillation of solutions for second-order nonlinear difference equations with nonlinear neutral term," Computers \& Mathematics with Applications, vol. 41, no. 12, pp. 1487-1494, 2001.

[30] Z. Zhang and Q. Li, "Oscillation theorems for second-order advanced functional difference equations," Computers \& Mathematics with Applications, vol. 36, no. 6, pp. 11-18, 1998.

[31] Z. Zhang and J. Zhang, "Oscillation criteria for second-order functional difference equations with "summation small" coefficient," Computers \& Mathematics with Applications, vol. 38, no. 1, pp. 25-31, 1999.

[32] M. Pinto and D. Sepúlveda, " $h$-asymptotic stability by fixed point in neutral nonlinear differential equations with delay," Nonlinear Analysis: Theory, Methods \& Applications, vol. 74, no. 12, pp. 3926-3933, 2011. 


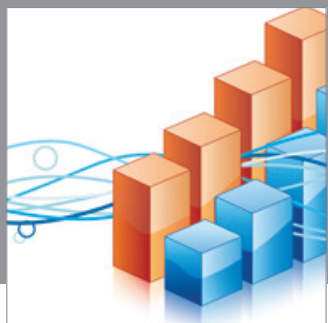

Advances in

Operations Research

mansans

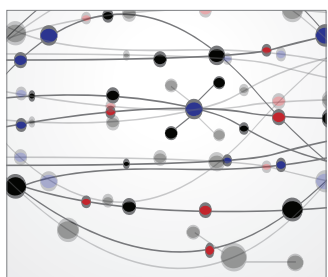

The Scientific World Journal
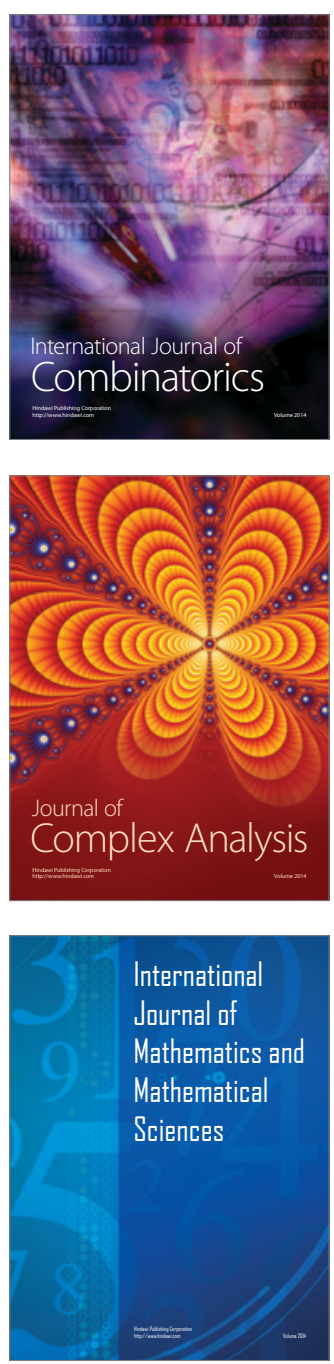
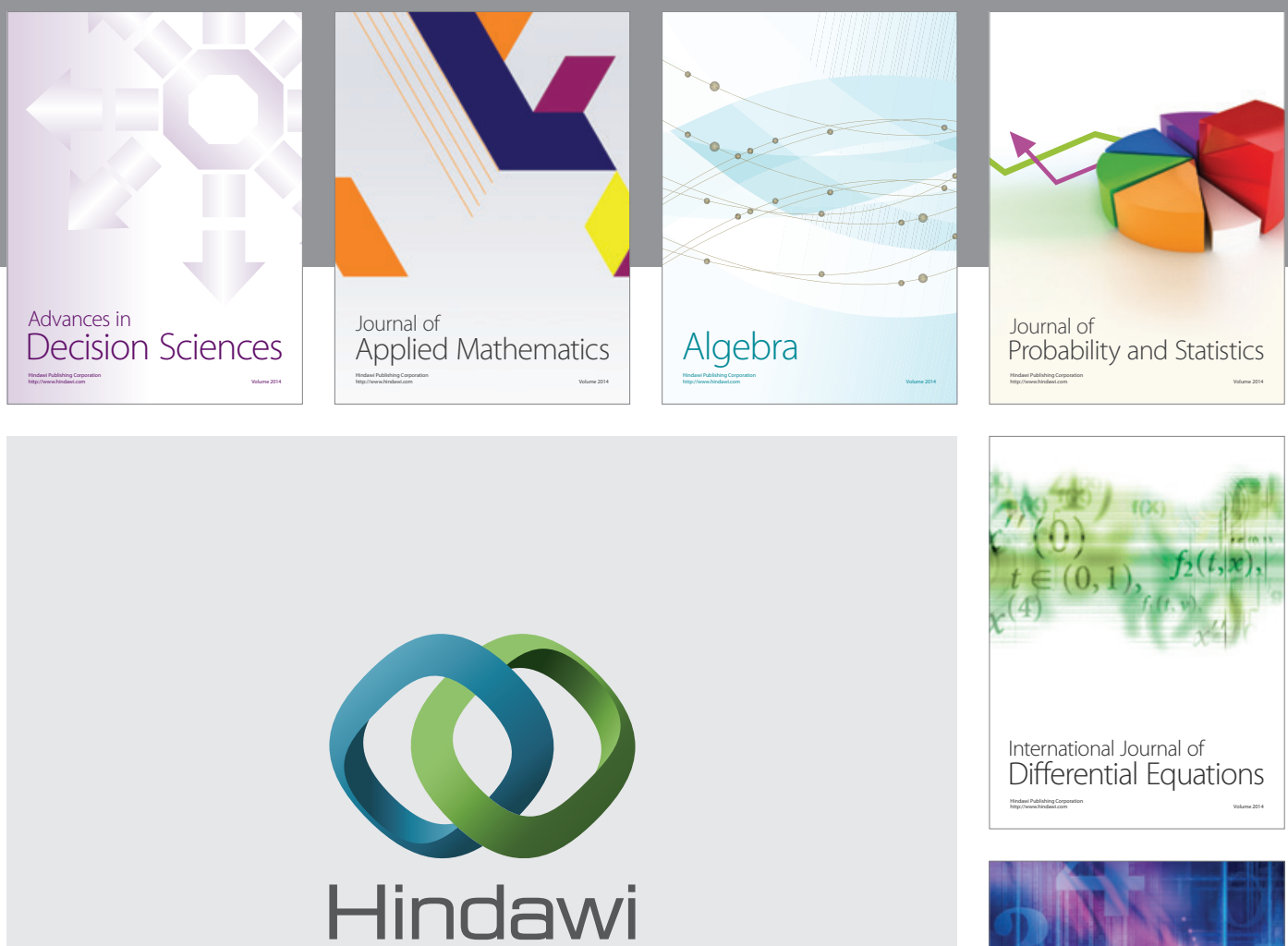

Submit your manuscripts at http://www.hindawi.com
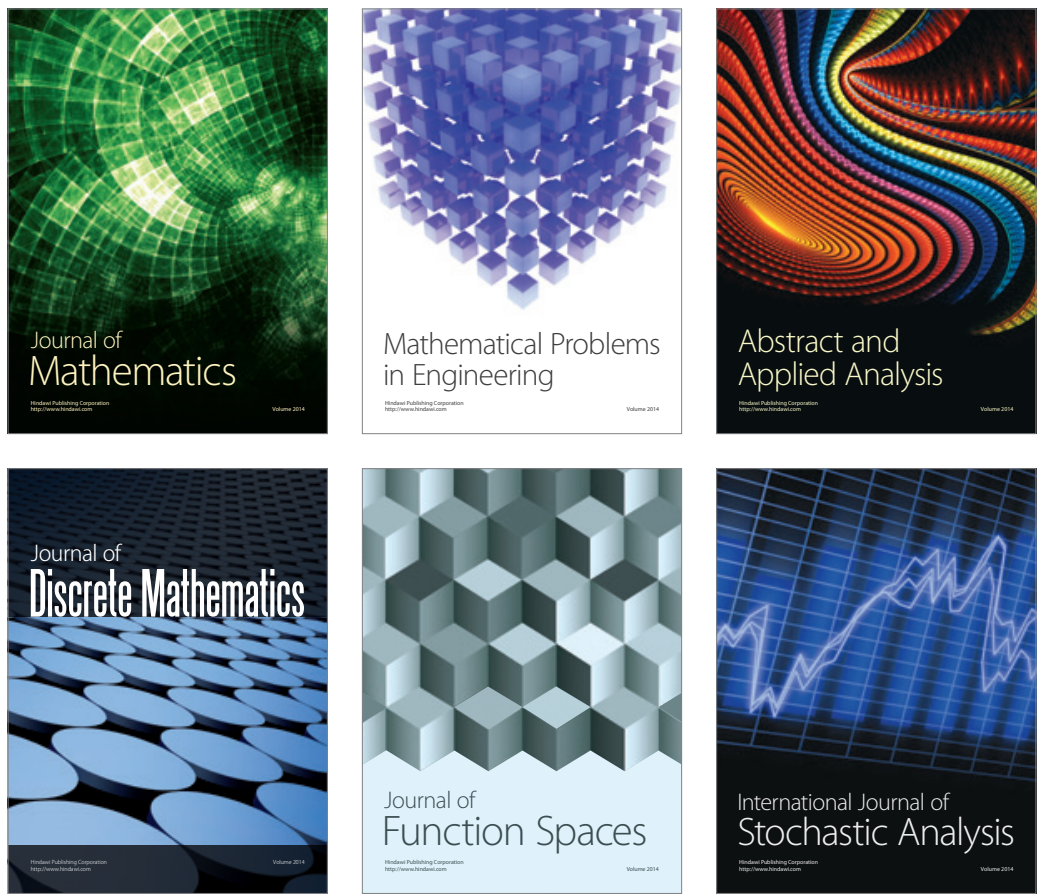

Journal of

Function Spaces

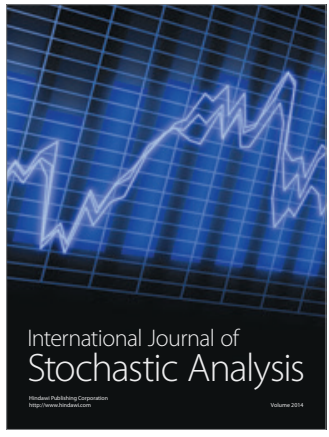

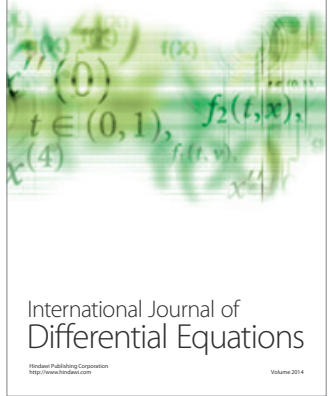
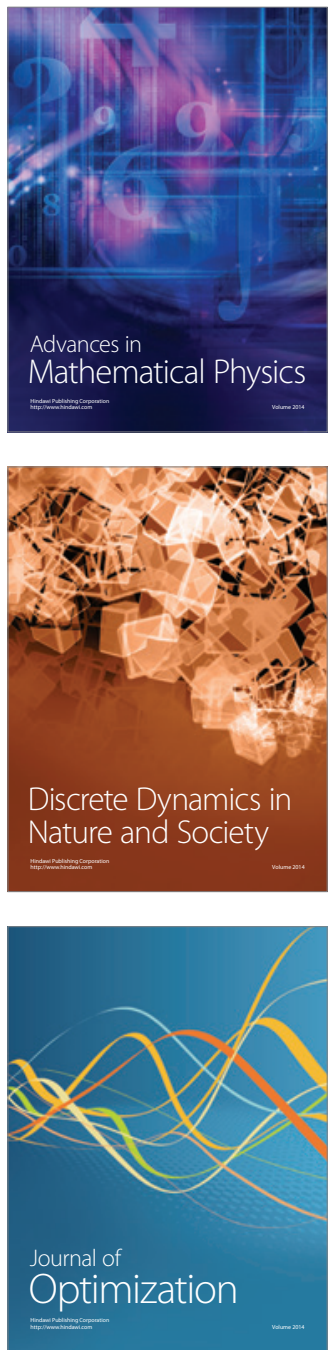